\title{
A Reference Discretization Strategy for the Numerical Solution of Physical Field Problems
}

\author{
CLAUDIO MATTIUSSI* \\ Clampco Sistemi-NIRLAB, AREA Science Park, \\ Padriciano 99, 34012 Trieste, ltaly
}

I. Introduction . . . . . . . . . . . . . . . . . . . . . . . 144

II. Foundations . . . . . . . . . . . . . . . . . . . . . . . . . . . . 147

A. The Mathematical Structure of Physical Field Theories . . . . . . . . . . 147

B. Geometric Objects and Orientation . . . . . . . . . . . . . . . . . . 150

I. Space-Time Objects . . . . . . . . . . . . . . . . . . . . . . . 155

C. Physical Laws and Physical Quantities . . . . . . . . . . . . . 157

1. Local and Global Quantities . . . . . . . . . . . . . . . . . . . . 158

2. Equations. . . . . . . . . . . . . . . . . . . . . . . . . . . . 159

D. Classification of Physical Quantities . . . . . . . . . . . . . . . . . . 163

1. Space-Time Viewpoint . . . . . . . . . . . . . . . . . . 165

E. Topological Laws . . . . . . . . . . . . . . . . . . . . . . . . . . 168

F. Constitutive Relations . . . . . . . . . . . . . . . . . . . . . . . . 172

1. Constitutive Equations and Discretization Error . . . . . . . . . . . . 175

G. Boundary Conditions and Sources . . . . . . . . . . . . . . . . . . . 176

H. The Scope of the Structural Approach . . . . . . . . . . . . . . . . . 177

III. Representations . . . . . . . . . . . . . . . . . . . . . . . . . . . . 183

A. Geometry . . . . . . . . . . . . . . . . . . . . . . . . 183

1. Cell Complexes . . . . . . . . . . . . . . . . . . . . . . . . . 184

2. Primary and Secondary Mesh . . . . . . . . . . . . . . . . . . 186

3. Incidence Numbers . . . . . . . . . . . . . . . . . . . . . . . . . . . . . . 188

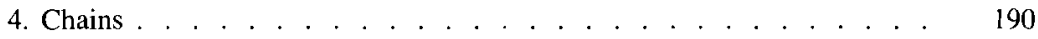

5. The Boundary of a Chain . . . . . . . . . . . . . . . 191

B. Fields . . . . . . . . . . . . . . . . . . . . . . . . . . . . . 193

1. Cochains . . . . . . . . . . . . . . . . . . 193

2. Limit Systems . . . . . . . . . . . . . . . . . . . . . . . 197

C. Topological Laws . . . . . . . . . . . . . . . . . . . . . . . . . 199

1. The Coboundary Operator . . . . . . . . . . . . . . . . . . . . 200

2. Properties of the Coboundary Operator . . . . . . . . . . . . . . . 202

3. Discrete Topological Equations . . . . . . . . . . . . . . . . . . . 204

D. Constitutive Relations . . . . . . . . . . . . . . . . . . . . . . . . 205

E. Continuous Representations . . . . . . . . . . . . . . 207

1. Differential Forms . . . . . . . . . . . . . . . . . . . . . . . . . 210

2. Weighted Integrals . . . . . . . . . . . . . . . . . . . . . . . . 211

${ }^{*}$ Current affiliation: Evolutionary and Adaptive Systems Team, Institute of Robotic Systems (ISR), Department of Micro-Engineering (DMT), Swiss Federal Institute of Technology (EPFL), $\mathrm{CH}-1015$ Lausanne, Switzerland. 
3. Differential Operators . . . . . . . . . . . . . . . . . . . . . 214

4. Spread Cells . . . . . . . . . . . . . . . . . . . . . . . . . . 217

5. Weak Form of Topological Laws . . . . . . . . . . . . . . . . . . . 220

IV. Methods . . . . . . . . . . . . . . . . . . . . . . . . . . . . . . . 222

A. The Reference Discretization Strategy . . . . . . . . . . . . . . . . . 222

1. Domain Discretization . . . . . . . . . . . . . . . 223

2. Topological Time Stepping . . . . . . . . . . . . . . . . . 225

3. Strategies for Constitutive Relations Discretization . . . . . . . . . . 231

4. Edge Elements and Field Reconstruction . . . . . . . . . . . . . . . 239

B. Finite Difference Methods . . . . . . . . . . . . . . . . . . . . . . 246

I. The Finite Difference Time-Domain Method . . . . . . . . . . . . . 246

2. The Support Operator Method . . . . . . . . . . . . . . . . . . . 252

3. Beyond the FDTD Method . . . . . . . . . . . . . . . . . . . . . 254

C. Finite Volume Methods . . . . . . . . . . . . . . . . . . . . . . . 255

1. The Discrete Surface Integral Method . . . . . . . . . . . . . . . 256

2. The Finite Integration Theory Method . . . . . . . . . . . . . . . . 260

D. Finite Element Methods . . . . . . . . . . . . . . . . . . . . . . . 264

1. Time-Domain Finite Element Methods . . . . . . . . . . . . . . . 267

2. Time-Domain Edge Element Method . . . . . . . . . . . . . . . . 269

3. Time-Domain Error-Based FE Method . . . . . . . . . . . . . . . 271

V. Conclusions . . . . . . . . . . . . . . . . . . . . . 273

VI. Coda . . . . . . . . . . . . . . . . . . . . . . . . . . . . 275

References . . . . . . . . . . . . . . . 276

\section{INTRODUCTION}

One of the fundamental concepts of mathematical physics is that of field; that is, naively speaking, of a spatial distribution of some mathematical object representing a physical quantity. The power of this idea lies in that it allows the modeling of a number of very important phenomena-for example, those grouped under the labels "electromagnetism," "thermal conduction," "fluid dynamics," and "solid mechanics," to name a few-and of the combinations thereof.

When the concept of field is used, a set of "translation rules" is devised, which transforms a physical problem belonging to one of the aforementioned domains-a physical field problem -into a mathematical one. The properties of this mathematical model of the physical problem-a model which usually takes the form of a set of partial differential or integrodifferential equations, supplemented by a set of initial and boundary conditions-can then be subjected to analysis in order to establish if the mathematical problem is well posed (Gustafsson et al., 1995). If the result of this inquiry is judged satisfactory, it is possible to proceed to the actual derivation of the solution, usually with the aid of a computer.

The recourse to a computer implies, however, a further step after the modeling step described so far, namely, the reformulation of the problem in discrete 
terms, as a finite set of algebraic equations, which are more suitable than a set of partial differential equations to the number-crunching capabilities of present-day computing machines. If this discretization step is made by starting from the mathematical problem in terms of partial differential equations, the resulting procedures can logically be called numerical methods for partial differential equations. This is indeed how the finite difference (FD), finite element (FE), finite volume (FV), and many other methods are often categorized. Finally, the system of algebraic equations produced by the discretization step is solved, and the result is interpreted from the point of view of the original physical problem.

More than 30 years ago, while considering the impact of the digital computer on mathematical activity, Bellman (1968) wrote

Much of the mathematical analysis that was developed over the eighteenth and nineteenth centuries originated in attempts to circumvent arithmetic. With our ability to do large-scale arithmetic ... we can employ simple, direct methods requiring much less old-fashioned mathematical training.... This situation by no mean implies that the mathematician has been dispossessed in mathematical physics. It does signify that he is urgently needed ... to transform the original mathematical problems to the stage where a computer can be utilized profitably by someone with a suitable scientific training.

... Good mathematics, like politics, is the art of the possible. Unfortunately, people quickly forget the origins of a mathematical formulation with the result that it soon acquires a life of its own. Its genealogy then protects it from scrutiny. Because the digital computer has so greatly increased our ability to do arithmetic, it is now imperative that we reexamine all the classical mathematical models of mathematical physics from the standpoints of both physical significance and feasibility of numerical solution. It may well turn out that more realistic descriptions are easier to handle conceptually and computationally with the aid of the computer. (pp. 44-45)

In this spirit, the present work describes an alternative to the classical partial differential equations-based approach to the discretization of physical field problems. This alternative is based on a preliminary reformulation of the mathematical model in a partially discrete form, which preserves as much as possible the physical and geometric content of the original problem, and is made possible by the existence and properties of a common mathematical structure of physical field theories (Tonti, 1975). The goal is to maintain the focus, both in the modeling step and in the discretization step, on the physics of the problem, thinking in terms of numerical methods for physical field problems, and not for a particular mathematical form (e.g., a partial differential equation) into which the original physical problem happens to be translated (Fig. 1). 


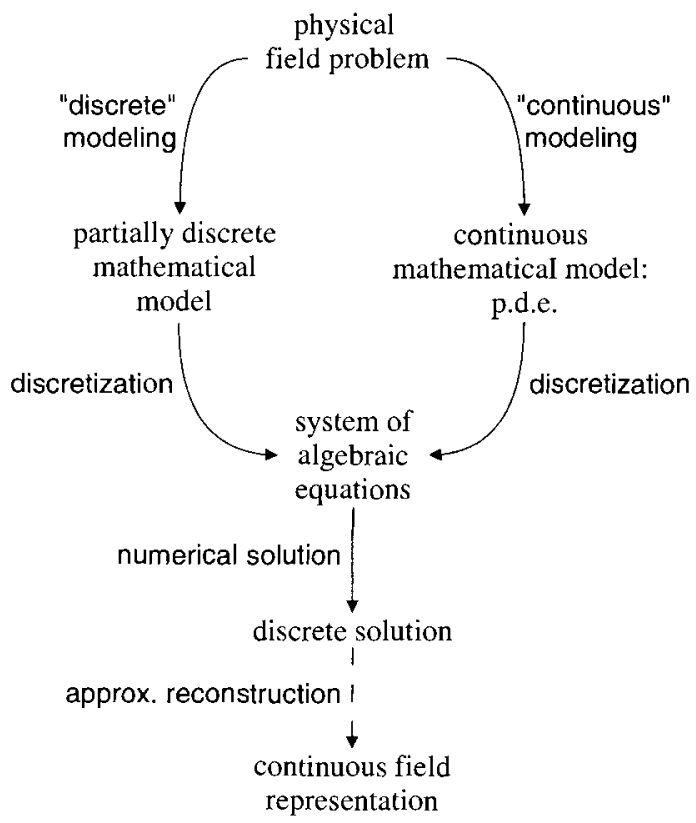

Figure 1. The alternative paths leading from a physical field problem to a system of algebraic equations. p.d.e., partial differential equation.

The advantages of this approach are various. First, it provides a unifying viewpoint for the discretization of physical field problems, which is valid for a multiplicity of theories. Second, by basing the discretization of the problems on the structural properties of the theory to which they belong, this approach gives discrete formulations which preserve many physically significant properties of the original problem. Finally, being based on very intuitive geometric and physical concepts, this approach facilitates both the analysis of existing numerical methods and the development of new ones. The present work considers both these aspects, introducing first a reference discretization strategy directly inspired by the results of the analysis of the structure of physical field theories. Then, a number of popular numerical methods for partial differential equations are considered, and their workings are compared with those of the reference strategy, in order to ascertain to what extent these methods can be interpreted as discretization methods for physical field problems.

The realization of this plan requires the preliminary introduction of the basic ideas of the structural analysis of physical field theories. These ideas are simple, but unfortunately they were formalized and given physically unintuitive names at the time of their first application, within certain branches of advanced 
mathematics. Therefore, in applying them to other fields, one is faced with the dilemma of inventing for these concepts new and, one would hope, more meaningful names, or maintaining the names inherited from mathematical tradition. After some hesitation, I chose to keep the original names, to avoid a proliferation of typically ephemeral new definitions and in consideration of the fact that there can be difficult concepts, not difficult names; we must try to clarify the former, not avoid the latter (Dolcher, 1978).

The intended audience for this article is wide. On the one hand, novices to the field of numerical methods for physical field problems will find herein a framework which will help them to intuitively grasp the common concepts hidden under the surface of a variety of methods and thus smooth the path to their mastery. On the other hand, the ideas presented should also prove helpful to the experienced numerical practitioner and to the researcher as additional tools that can be applied to the evaluation of existing methods and the development of new ones.

Finally, it is worth remembering that the result of the discretization must be subjected to analysis also, in order to establish its properties as a new mathematical problem, and to measure the effects of the discretization on the solution when it is compared with that of nondiscrete mathematical models. This further analysis will not be dealt with here, the emphasis being on the unveiling of the common discretization substratum for existing methods, the convergence, stability, consistency, and error analyses of which abound in the literature.

\section{FOUNDATIONS}

\section{A. The Mathematical Structure of Physical Field Theories}

It was mentioned in the Introduction that the approach to the discretization that will be presented in this work is based on the observation that physical field theories possess a common structure. Let us, therefore, start by explaining what we mean when we talk of the structure of a physical theory.

It is a common experience that exposure to more than one physical field theory (e.g., thermal conduction and electrostatics) aids the comprehension of each single one and facilitates the quick grasping of new ones. This occurs because there are easily recognizable similarities in the mathematical formulation of theories describing different phenomena, which permit the transfer of intuition and imageries developed for more familiar cases to unfamiliar realms. ${ }^{*}$ Building in a systematic way on these similarities, one can fill a correspondence

${ }^{*}$ One may say that this is the essence of explanation (i.e., the mapping of the unexplained on something that is considered obvious). 
table that relates physical quantities and laws playing a similar role within different theories. Usually we say that there are analogies between these theories. These analogies are often reported as a trivial, albeit useful curiosity, but some scholars have devoted considerable efforts to unveiling their origin and meaning. In these scholars' quest, they have discovered that these similarities can be traced to the common geometric background upon which the "physics" is built. In the book that, building on a long tradition, took these enquiries almost to their present state, Tonti (1975) emphasized the following:

- The existence within physical theories of a natural association of many physical quantities, with geometric objects in space and space-time*

- The necessity to consider as oriented the geometric objects to which physical quantities are associated

- The existence of two kinds of orientation for these geometric objects

- The primacy and priority, in the foundation of each theory, of global physical quantities associated with geometric objects, over the corresponding densities

From this set of observations there follows naturally a classification of physical quantities, based on the type and kind of orientation of the geometric object with which they are associated. The next step is the consideration of the relations held between physical quantities within each theory. Let us call them generically the physical laws. From our point of view, the fundamental observation in this context relates to

- The existence within each theory of a set of intrinsically discrete physical laws

These observations can be given a graphical representation as follows. A classification diagram for physical quantities is devised, with a series of "slots" for the housing of physical quantities, each slot corresponding to a different kind of oriented geometric object (see Figs. 7 and 8). The slots of this diagram can be filled for a number of different theories. Physical laws will be represented in this diagram as links between the slots housing the physical quantities (see Fig. 17). The classification diagram of physical quantities, complemented by the links representing physical laws, will be called the factorization diagram of the physical field problem, to emphasize its role in singling out the terms in the governing equations of a problem, according to their mathematical and physical properties.

The classification and factorization diagrams will be used extensively in this work. They seem to have been first introduced by Roth (see the discussion

\footnotetext{
${ }^{*}$ For the time being, we give the concept of oriented geometric object an intuitive meaning (points, and sufficiently regular lines, surfaces, volumes, and hypervolumes, along with time instants and time intervals).
} 
in Bowden, 1990, who calls them Roth's diagrams). Branin (1966) used a modified version of Roth's diagrams, calling them transformation diagrams. Tonti $(1975,1976 \mathrm{a}, 1976 \mathrm{~b}, 1998)$ refined and used these diagrams-which he called classification schemes-as the basic representational tool for the analysis of the formal structure of physical theories. We will refer here to this last version of the diagrams, which were subsequently adopted by many authors with slight graphical variations and under various names (Baldomir and Hammond, 1996; Bossavit, 1998a; Palmer and Shapiro, 1993; Oden and Reddy, 1983) and for which the name Tonti diagrams was suggested.*

The Tonti classification and factorization diagrams are an ideal starting point for the discretization of a field problem. The association of physical quantities with geometric objects gives a rationale for the construction of the discretization meshes and the association of the variables to the constituents of the meshes, whereas singling out in the diagram the intrinsically discrete terms of the field equation permits us both to pursue the direct discrete rendering of these terms and to focus on the discretization effort with the remaining terms.

Having found this common starting point for the discretization of field problems, one might be tempted to adopt a very abstract viewpoint, based on a generic field theory, with a corresponding generic terminology and factorization diagram. However, although many problems share the same structure of the diagram, there are classes of theories whose diagrams differ markedly and consequently a generic diagram would be either too simple to encompass all the cases or too complicated to work with. For this reason we are going to proceed in concrete terms, selecting a model field theory and referring mainly to it, in the belief that this could aid intuition, even if the reader's main interest is in a different field. Considering the focus of the series in which this article appears, electromagnetism was selected as the model theory. Readers having another background can easily translate what follows by comparing the factorization diagram for electromagnetism with that of the theory they are interested in. To give a feeling of what is required for the development of the factorization diagram for other theories, we discuss the case of heat transfer, thought of as representative of a class of scalar transport equations.

It must be said that there are still issues that wait to be clarified in relation to the factorization diagrams and the mathematical structure of physical theories. This is true in particular for some issues concerning the position of energy quantities within the diagrams and the role of orientation with reference to

\footnotetext{
*In fact, the diagrams used in this work (and in Mattiussi, 1997) differ from those originally conceived by Tonti in their admitting only cochains within the slots, whereas the latter had chains in some slots and cochains in others (depending on the kind of orientation of the subjacent geometric object). This difference reflects our advocating the use of the chain-cochain pair to distinguish the discrete representation of the geometry (which is always made in terms of chains) from that of the fields (which is always based on cochains).
} 
time. Luckily this touches only marginally on the application of the theory to the discretization of physical problems finalized to their numerical solution.

\section{B. Geometric Objects and Orientation}

The concept of geometric object is ubiquitous in physical field theories. For example, in the theory of thermal conduction the heat balance equation links the difference between the amount of heat contained inside a volume $V$ at the initial and final time instants $T_{i}$ and $T_{f}$ of a time interval $I$, to the heat flowing through the surface $S$, which is the boundary of $V$, and to the heat produced or absorbed within the volume during the time interval. In this case, $V$ and $S$ are geometric objects in space, whereas $I, T_{i}$, and $T_{f}$ are geometric objects in time. The combination of a space and a time object (e.g., the surface $S$ considered during the time interval $I$, or the volume $V$ at the time instant $T_{i}$, or $T_{f}$ ) gives a spacetime geometric object. These examples show that by "geometric object" we mean the points and the sufficiently well-behaved lines, surfaces, volumes, and hypervolumes contained in the domain of the problem, and their combination with time instants and time intervals. This somewhat vague definition will be substituted later by the more detailed concept of the $p$-dimensional cell.

The preceding example also shows that each mention of an object comes with a reference to its orientation. To write the heat balance equation, we must specify if the heat flowing out of a volume or that flowing into it is to be considered positive. This corresponds to the selection of a preferred direction through the surface. ${ }^{*}$ Once this direction is chosen, the surface is said to have been given external orientation, where the qualifier "external" hints at the fact that the orientation is specified by means of an arrow that does not lie on the surface. Correspondingly, we will call internal orientation of a surface that which is specified by an arrow that lies on the surface and that specifies a sense of rotation on it (Fig. 2). Note that the idea of internal orientation for surfaces is seldom mentioned in physics but is very common in everyday objects and in mathematics (Schutz, 1980). For example, a knob that must be rotated counterclockwise to ensure a certain effect is usually designed with a suitable curved arrow drawn on its surface, and in plane affine geometry, the ordering of the coordinate axes corresponds to the choice of a sense of rotation on the plane and defines the orientation of the space.

\footnotetext{
${ }^{*}$ Of course it must be possible to assign such a direction consistently, which is true if the geometric object is orientable (Schutz, 1980), as we will always suppose to be the case. Once the selection is made, the object acquires a new status. As pointed out by MacLane (1986): "A plane with orientation is really not the same object as one without. The plane with an orientation has more structure-namely, the choice of the orientation" (p. 84).
} 


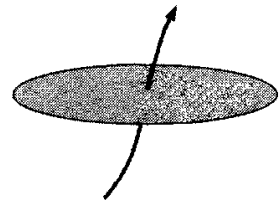

(a)

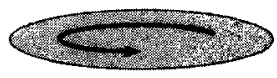

(b)

FIGURE 2. (a) External and (b) internal orientations for surfaces.

In fact, all geometric objects can be endowed with two kinds of orientations but, for historical reasons, almost no mention of this distinction survives in physics. ${ }^{*}$ Since both kinds of orientation are needed in physics, we will show how to build the complete orientation apparatus. We will start with internal orientation, using the preceding affine geometry example as inspiration. An $n$-dimensional affine space is oriented by fixing an order of the coordinate axes: this, in the three-dimensional case, corresponds to the choice of a screw-sense, or that of a vortex; in the two-dimensional case, to the choice of a sense of rotation on the plane; and in the one-dimensional case, to the choice of a sense (an arrow) along the line. These images can be extended to geometric objects. Therefore, the internal orientation of a volume is given by a screw-sense; that of a surface, by a sense of rotation on it; and that of a line, by a sense along it (see Fig. 5).

Before we proceed further, it is instructive to consider an example of a physical quantity that, contrary to common belief, is associated with internally oriented surfaces: the magnetic flux $\phi$. This association is a consequence of the invariance requirement of Maxwell's equations for improper coordinate transformations; that is, those that invert the orientation of space, transforming a right-handed reference system into a left-handed one. Imagine an experimental setup to probe Faraday's law, for example, verifying the link between the magnetic flux $\phi$ "through" a disk $S$ and the circulation $\mathcal{U}$ of the electric field intensity $\mathbf{E}$ around the loop $\Gamma$ which is the border of $S$. If we suppose, as is usually the case, that the sign of $\phi$ is determined by a direction through the disk, and that of $\mathcal{U}$ by the choice of a sense around the loop, a mirror reflection through a plane parallel to the disk axis changes the sign of $\mathcal{U}$ but not that of $\phi$. Usually the incongruence is avoided by using the right-hand rule to define $\mathbf{B}$ and invoking for it the status of axial vector (Jackson, 1975). In other words, we are told that for space reflections, the sense of the "arrow" of the $\mathbf{B}$ vector

*However, for example, Maxwell (1871) was well aware of the necessity within the context of electromagnetism of at least four kinds of mathematical entities for the correct representation of the electromagnetic field (entities referred to lines or to surfaces and endowed with internal or with external orientation). 


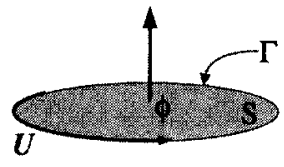

(a)

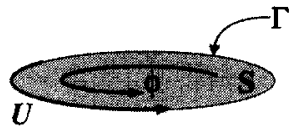

(b)

FIGURE 3. Orientational issues in Faraday's law. The intervention of the right-hand rule, required in the classical version (a), can be avoided by endowing both geometric objects $\Gamma$ and $S$ with the same kind of orientation (b).

does not count; only the right-hand rule does. It is, however, apparent that for the invariance of Faraday's law to hold true without such tricks, all we have to do is either to associate $\phi$ with internally oriented surfaces and $\mathcal{U}$ with internally oriented lines, or to associate $\phi$ with externally oriented surfaces and $\mathcal{U}$ with lines oriented by a sense of rotation around them (i.e., externally oriented lines, as will soon be clear). Since the effects of an electric field act along the field lines and not around them, the first option seems preferable (Schouten, 1989; Fig. 3).

This example shows that the need for the right-hand rule is a consequence of our disregarding the existence of two kinds of orientation. This attitude seems reasonable in physics as we have become accustomed to it in the course of our education, but consider that if it were applied systematically to everyday objects, we would be forced to glue an arrow pointing outward from the aforementioned knob, and to accompany it with a description of the right-hand rule. Note also that the difficulties in the classical formulation of Faraday's law stem from the impossibility of comparing directly the orientation of the surface with that of its boundary, when the surface is externally oriented and the bounding line is internally oriented. In this case, "directly" means "without recourse to the right-hand rule" or similar tricks. The possibility of making this direct comparison is fundamental for the correct statement of many physical laws. This comparison is based on the idea of an orientation induced by an object on its boundary. For example, the sense of rotation that internally orients a surface induces a sense of rotation on its bounding curve, which can be compared with the sense of rotation which orients the surface internally. The same is true for the internal orientation of volumes and of their bounding surfaces. The reader can check that the direct comparison is indeed possible if the object and its boundary are both endowed with internal orientation as defined previously for volumes, surfaces, and lines. However, this raises an interesting issue, since our list of internally oriented objects does not so far include points, which nevertheless form the boundary of a line. To make inner orientation a coherent system, we must, therefore, define internal orientations for points (as in algebra we extend the definition of the $n$th power of a number to include the case $n=0$ ). This can be done by means of a pair of symbols 


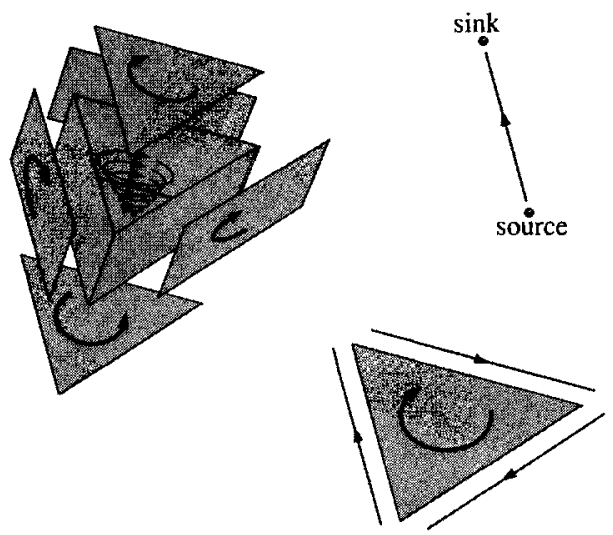

FIGURE 4. Each internally oriented geometric object induces an internal orientation on the objects that constitute its boundary.

meaning "inward" and "outward" (e.g., defining the point as a sink or a source, or drawing arrows pointing inward or outward), for these images are directly comparable with the internal orientation of a line which starts or ends with the point (Fig. 4).

This completes our definition of internal orientation for geometric objects in three-dimensional space, which we will indicate with the terms $P, L, S$, and $V$. Let us now tackle the definition of external orientation for the same objects. We said before that in three-dimensional space the external orientation of a surface is given, specifying what turned out to be the internal orientation of a line which does not lie on the surface. This is a particular case of the very definition of external orientation: in an $n$-dimensional space, the external orientation of a $p$-dimensional object is specified by the internal orientation of a dual $(n-p)$ dimensional geometric object (Schouten, 1989). Hence, in three-dimensional space, external orientation for a volume is specified by an inward or outward symbol; for a surface, it is specified by a direction through it; for a line, by a sense of rotation around it; for a point, by the choice of a screw-sense. To distinguish internally oriented objects from externally oriented ones, we will add a tilde to the terms for the latter, thus writing $\tilde{P}, \tilde{L}, \tilde{S}$, and $\tilde{V}$ for externally oriented points, lines, surfaces, and volumes, respectively (Fig. 5).

The definition of external orientation in terms of internal orientation has many consequences. First, contrary to internal orientation, which is a combinatorial concept ${ }^{*}$ and does not change when the dimension of the embedding

*For example, a line can be internally oriented by selecting a permutation class (an ordering) of two distinct points on it, which become three nonaligned points for a surface, four noncoplanar points for a volume, and so on. 


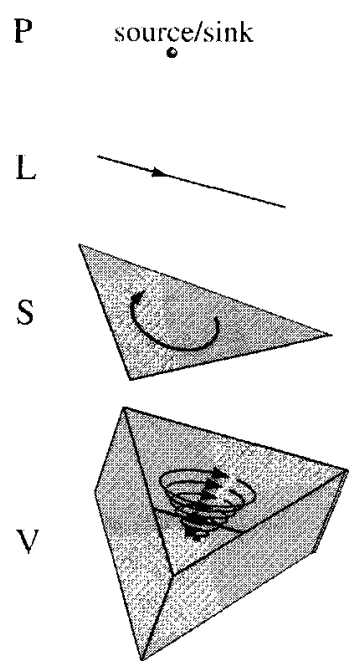

(a)

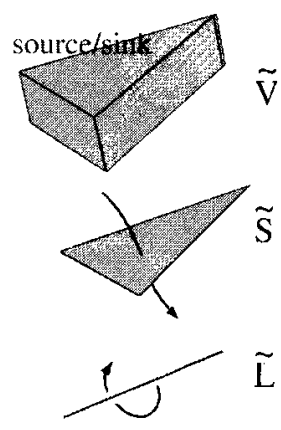

$\tilde{\mathrm{V}}$

\section{$\tilde{S}$}

\section{$\tilde{\mathrm{L}}$}

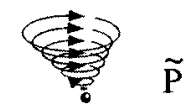

(b)

FIgURE 5. (a) Internal and (b) external orientations for geometric objects in threedimensional space. The disposition of objects reflects the pairing of reciprocally dual geometric objects.

space varies, external orientation depends on the dimension. For example, external orientation for a line in two-dimensional space is assigned by a direction through it and not around it as in three-dimensional space.* Another consequence is the inheritance from internal orientation of the possibility of comparing the orientation of an object with that of its boundary, when both are endowed with external orientation. This implies once again the concept of induced orientation, applied in this case to externally oriented objects (Fig. 6).

The duality of internal and external orientation gives rise to another important pairing, that between dual geometric objects; that is, between pairs of geometric objects that in an $n$-dimensional space have dimensions $p$ and $(n-p)$, respectively, and have differents kinds of orientation (Fig. 5). Note that also in this case the orientation of the objects paired by the duality can be directly compared. However, contrary to what happens for a geometric object and its boundary, the objects have different kinds of orientation. In the context of the mathematical structure of physical theories, this duality plays an

"Note, however, that the former can be considered the "projection" onto the surface of the latter. 


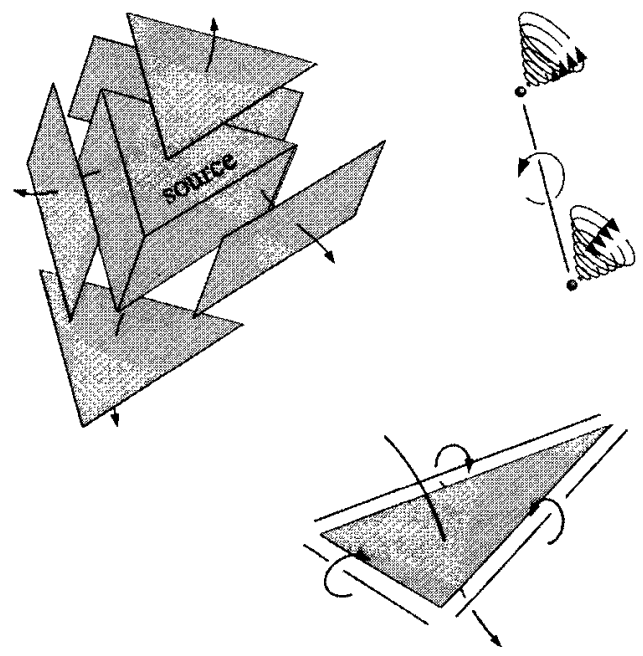

FIGURE 6. Each externally oriented geometric object induces an external orientation on the objects that constitute its boundary.

important role; for example, it is used in the definition of energy quantities and it accounts for some important adjointness relationships between differential operators.

We have now at our disposal all the elements required for the construction of a first version-referring to the objects of three-dimensional space-of the classification diagram of physical quantities. As anticipated, it consists of a series of slots for the housing of physical quantities, each slot corresponding to an oriented geometric object. As a way to represent graphically the distinction between internal and external orientation, the slots of the diagram are subdivided between two columns. So that the important relationship represented by duality is reflected, these two columns-for internal and external orientation, respectively-are reversed with respect to each other, which thus makes dual objects row-adjacent (Fig. 7).

\section{Space-Time Objects}

In the heat balance example that opens this section, it was shown how geometric objects in space, time, and space-time make their appearance in the foundation of a physical theory. Until now, we have focused on objects in space; let us extend our analysis to space-time objects.

If we adopt a strict space-time viewpoint-that is, if we consider space and time as one, and our objects as $p$-dimensional objects in a generic 


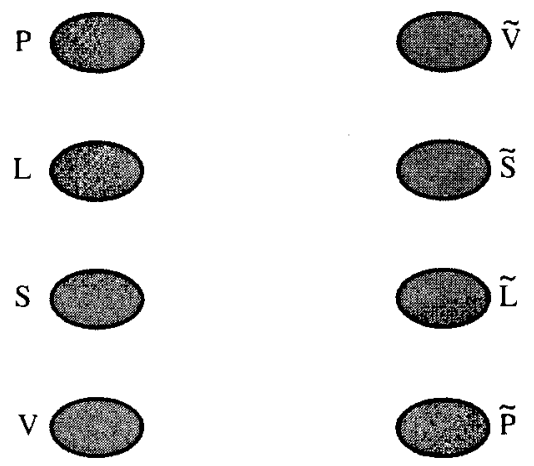

FIGURE 7. The Tonti classification diagram of physical quantities in three-dimensional space. Each slot is referred to an oriented geometric object; that is, points $P$, lines $L$, surfaces $S$, and volumes $V$. The left column is devoted to internally oriented objects, and the right column to externally oriented ones. The slots are paired horizontally so as to reflect the duality of the corresponding objects.

four-dimensional space-the extension from space to space-time requires only that we apply to the four-dimensional case the definitions given previously for oriented geometric objects. However, one cannot deny that in all practical cases (i.e., if a reference frame has to be meaningful for an actual observer) the time coordinate is clearly distinguishable from the spatial coordinates. Therefore, it seems advisable to consider, in addition to space-time objects per se, the space-time objects considered as Cartesian products of a space object by a time object.

Let us list these products. Time can house zero- and one-dimensional geometric objects: time instants $T$ and time intervals $I$. We can combine these time objects with the four space objects: points $P$, lines $L$, surfaces $S$, and volumes $V$. We obtain thus eight combinations that, considering the two kinds of orientation they can be endowed with, give rise to the 16 slots of the space-time classification diagram of physical quantities (Tonti, 1976b; Fig. 8). Note that the eight combinations correspond, in fact, to five space-time geometric objects (e.g., a space-time volume can be obtained as a volume in space considered at a time instant, that is, as the topological product $V \times T$, or as a surface in space considered during a time interval, which corresponds to $S \times I$ ). This is reflected within the diagram by the sharing of a single slot by the combinations corresponding to the same oriented space-time object. To distinguish space-time objects from merely spatial ones, we will use the symbols $\mathcal{P}, \mathcal{L}, \mathcal{S}, \mathcal{V}$, and $\mathcal{H}$ for the former and the symbols $P, L, S$, and $V$ for the latter. As usual, a tilde will signal external orientation. 

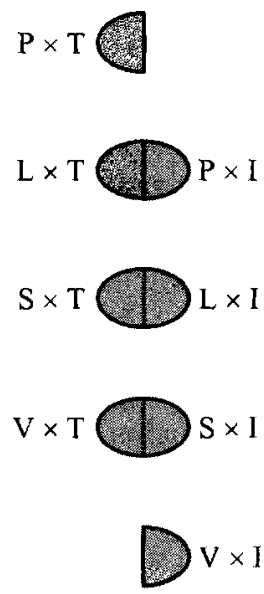
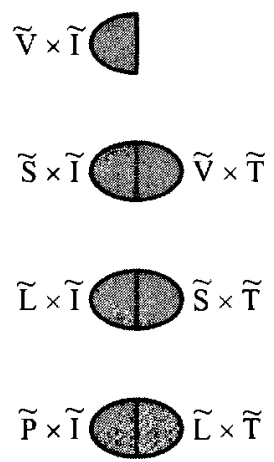

D $\widetilde{\mathrm{P}} \times \widetilde{\mathrm{T}}$

FIGURE 8. The Tonti space-time classification diagram of physical quantities. Each slot is referred to an oriented space-time geometric object, which is thought of as obtained in terms of a product of an object in space by an object in time. The space objects are those of Figure 7. The time objects are time instants $T$ and time intervals $I$. This diagram can be redrawn with the slots referring to generic space-time geometric objects; that is, points $\mathcal{P}$, lines $\mathcal{L}$, surfaces $\mathcal{S}$, volumes $\mathcal{V}$, and hypervolumes $\mathcal{H}$ (see Fig. 11).

\section{Physical Laws and Physical Quantities}

In the previous sections, we have implicitly defined a physical quantity (the heat content, the heat flow, and the heat production, in the heat transfer example) as an entity appearing within a physical field theory, which is associated with one (and only one) kind of oriented geometric object. Strictly speaking, the individuation within a physical theory of the actual physical quantities and the attribution of the correct association with oriented geometric objects should be based on an analysis of the formal properties of the mathematical entities that appear in the theory (e.g., considering the dimensional properties of those entities and their behavior with respect to coordinate transformations). Given that formal analyses of this kind are available in the literature (Post, 1997; Schouten, 1989; Truesdell and Toupin, 1960), the approach within the present work will be more relaxed. To fill in the classification diagram of the physical quantities of a theory, we will look first at the integrals which appear within the theory, focusing our attention on the integration domains in space and time. This will give us a hint about the geometric object that a quantity is associated with. The attribution of orientation to these objects will be based on heuristic considerations deriving from the following fundamental property: the sign of a global quantity associated with a geometric object changes when 
the orientation of the object is inverted. Further hints would be drawn from physical effects and the presence of the right-hand rule in the traditional definition of a quantity, as well as from the global coherence of the orientation system thus defined. The reader can find in Tonti (1975) an analysis based on a similar rationale, applied to a large number of theories, accompanied by the corresponding classification and factorization diagrams.

\section{Local and Global Quantities}

By their very definition, our physical quantities are global quantities, for they are associated with macroscopic space-time domains. This complies with the fact that actual field measurements are always performed on domains having finite extension. When local quantities (densities and rates) can be defined, it is natural to make them inherit the association with the oriented geometric object of the corresponding global quantity. However, it is apparent that the familiar tools of vector analysis do not allow this association to be represented. This causes a loss of information in the transition from the global to the local representation, when ordinary scalars and vectors are used. For example, from the representation of magnetic flux density with the vector field $\mathbf{B}$, no hint at internally oriented surfaces can be obtained, nor can an association to externally oriented volumes be derived from the representation of charge density with the scalar field $\rho$. Usually the association with geometric objects (but not the distinction between internal and external orientations) is reinserted while one is writing integral relations, by means of the "differential term," so that we write, for example,

$$
\int_{S} \mathbf{B} \cdot d \mathrm{~s}
$$

and

$$
\int_{V} \rho d v
$$

However, given the presence of the integration domains $S$ and $V$, which accompany the integration signs, the terms $d \mathbf{s}$ and $d v$ look redundant. It would be better to use a mathematical representation that refers directly to the oriented geometric object that a quantity is associated with. Such a representation exists within the formalism of ordinary and twisted differential forms (Burke, 1985; de Rham, 1931). Within this formalism, the vector field $\mathbf{B}$ becomes an ordinary 2-form $b^{2}$ and the scalar field $\rho$ a twisted 3 -form $\tilde{\rho}^{3}$, as follows:

$$
\begin{aligned}
\mathbf{B} & \Rightarrow b^{2} \\
\rho & \Rightarrow \tilde{\rho}^{3}
\end{aligned}
$$


The symbols $b^{2}$ and $\tilde{\rho}^{3}$ explicitly refer to the fact that magnetic induction and charge density are associated with (and can be integrated only on) internally oriented two-dimensional domains and externally oriented three-dimensional domains, respectively.

Thus, everything seems to conspire for an early adoption of a representation in terms of differential forms. We prefer, however, to delay this step in order to show first how the continuous representation tool they represent can be founded on discrete concepts. Waiting for the suitable discrete concepts to be available, we will temporarily stick to the classical tools of vector calculus. In the meantime, the only concession to the differential-form spirit will be the systematic dropping of the "differential" under the integral sign, so that we write, for example,

$$
\int_{S} \mathbf{B}
$$

and

$$
\int_{\tilde{V}} \rho
$$

instead of Eqs. (1) and (2).

\section{Equations}

After the introduction of the concept of oriented geometric objects, the next step would ideally be the discussion of the association of the physical quantities of the field theory (in our case, electromagnetism) with the objects. This would parallel the typical development of physical theories, in which the discovery of quantities upon which the phenomena of the theory may be conceived to depend precedes the development of the mathematical relations that link those quantities in the theory (Maxwell, 1871). It turns out, however, that the establishment of the association between physical quantities and geometric objects is based on the analysis of the equations appearing in the theory itself. In particular, it is expedient to list all pertinent equations for the problem considered, and isolate a subset of them, which represent physical laws lending themselves naturally to a discrete rendering, for these clearly expose the correct association. We start, therefore, by listing the equations of electromagnetism. We will first give a local rendition of all the equations, even of those that will eventually turn out to have an intrinsically discrete nature, since this is the form that is typically considered in mathematical physics.

The first pair of electromagnetic equations that we consider represent in local form Gauss's law for magnetic flux [Eq. (7)] and Faraday's induction 
law [Eq. (8)]:

$$
\begin{array}{r}
\operatorname{div} \mathbf{B}=0 \\
\operatorname{curl} \mathbf{E}+\frac{\partial \mathbf{B}}{\partial t}=\mathbf{0}
\end{array}
$$

where $\mathbf{B}$ is the magnetic flux density and $\mathbf{E}$ is the electric field intensity. We will show next that these equations have a counterpart in the law of charge conservation [Eq. (9)]:

$$
\operatorname{div} \mathbf{J}+\frac{\partial \rho}{\partial t}=0
$$

where $\mathbf{J}$ is the electric current density and $\rho$ is the electric charge density. Similarly, Eqs. (10) and (11), which define the scalar potential $V$ and the vector potential A,

$$
\begin{array}{r}
\operatorname{curl} \mathbf{A}=\mathbf{B} \\
-\operatorname{grad} V-\frac{\partial \mathbf{A}}{\partial t}=\mathbf{E}
\end{array}
$$

are paralleled by Gauss's law of electrostatics [Eq. (12)] and MaxwellAmpère's law [Eq. (13)] — where $\mathbf{D}$ is the electric flux density and $\mathbf{H}$ is the magnetic field intensity-which close the list of differential statements:

$$
\begin{array}{r}
\operatorname{div} \mathbf{D}=\rho \\
\operatorname{curl} \mathbf{H}-\frac{\partial \mathbf{D}}{\partial t}=\mathbf{J}
\end{array}
$$

Finally, we have a list of constitutive equations. A very general form for the case of electromagnetism, accounting for most material behaviors, is

$$
\begin{aligned}
& \mathbf{D}(\mathbf{r}, t)=\int_{t_{0}}^{t} \int_{D} F_{\varepsilon}\left(\mathbf{E}, \mathbf{r}^{\prime}, \tau\right) \\
& \mathbf{B}(\mathbf{r}, t)=\int_{t_{0}}^{t} \int_{D} F_{\mu}\left(\mathbf{H}, \mathbf{r}^{\prime}, \tau\right) \\
& \mathbf{J}(\mathbf{r}, t)=\int_{t_{0}}^{t} \int_{D} F_{\sigma}\left(\mathbf{E}, \mathbf{r}^{\prime}, \tau\right)
\end{aligned}
$$

but, typically, the purely local relations

$$
\begin{aligned}
& \mathbf{D}(\mathbf{r}, t)=f_{\varepsilon}(\mathbf{E}, \mathbf{r}, t) \\
& \mathbf{B}(\mathbf{r}, t)=f_{\mu}(\mathbf{H}, \mathbf{r}, t) \\
& \mathbf{J}(\mathbf{r}, t)=f_{\sigma}(\mathbf{E}, \mathbf{r}, t)
\end{aligned}
$$


or the even simpler relations

$$
\begin{aligned}
& \mathbf{D}(\mathbf{r})=\varepsilon(\mathbf{r}) \mathbf{E}(\mathbf{r}) \\
& \mathbf{B}(\mathbf{r})=\mu(\mathbf{r}) \mathbf{H}(\mathbf{r}) \\
& \mathbf{J}(\mathbf{r})=\sigma(\mathbf{r}) \mathbf{E}(\mathbf{r})
\end{aligned}
$$

adequately represent most actual material behaviors.

We will now consider all these equations, aiming at their exact rendering in terms of global quantities. Integrating Eqs. (7) through (13) on suitable spatial domains, writing $\partial D$ for the boundary of a domain $D$, and making use of Gauss's divergence theorem and Stokes's theorem, we obtain the following integral expressions:

$$
\begin{aligned}
\int_{\partial V} \mathbf{B} & =\int_{V} 0 \\
\int_{\partial S} \mathbf{E}+\frac{d}{d t} \int_{S} \mathbf{B} & =\int_{S} \mathbf{0} \\
\int_{\partial \tilde{V}} \mathbf{J}+\frac{d}{d t} \int_{\tilde{V}} \rho & =\int_{\tilde{V}} 0 \\
\int_{\partial S} \mathbf{A} & =\int_{S} \mathbf{B} \\
-\int_{\partial L} V-\frac{d}{d t} \int_{L} \mathbf{A} & =\int_{L} \mathbf{E} \\
\int_{\partial \tilde{V}} \mathbf{D} & =\int_{\tilde{V}} \rho \\
\int_{\partial \tilde{S}} \mathbf{H}-\frac{d}{d t} \int_{\tilde{S}} \mathbf{D} & =\int_{\tilde{S}} \mathbf{J}
\end{aligned}
$$

Note that in Eqs. (23), (24), and (25) we have integrated the null term on the right-hand side. This was done in consideration of the fact that the corresponding equations assert the vanishing of some kind of physical quantity, and we must investigate what kind of association it has. Moreover, in Eqs. (25), (28), and (29) we added a tilde to the symbol of the integration domains. These are the domains which will turn out later to have external orientation. 
In Eqs. (24), (25), (27), and (29) a time derivative remains. A further integration can be performed on a time interval $I=\left[T_{1}, T_{2}\right]$ as a way to eliminate this residual derivative. For example, Eq. (24) becomes

$$
\left.\int_{T_{1}}^{T_{2}} \int_{\partial S} \mathbf{E}+\int_{S} \mathbf{B}\right]_{T_{1}}^{T_{2}}=\int_{T_{1}}^{T_{2}} \int_{S} \mathbf{0}
$$

We adopt a more compact notation, which uses $I$ for the time interval. Moreover, we will consider as an "integral on time instants," a term evaluated at that instant, according to the following symbolism:

$$
\int_{S} \cdot=\int_{S} \cdot \int_{T}^{\stackrel{\text { def }}{=}} \int_{T} \int_{S}
$$

Correspondingly, since the initial and final instants of a time interval $I$ are actually the boundary $\partial I$ of $I$, we write boundary terms as follows:

$$
\left.\int_{S} \cdot\right]_{T_{1}}^{T_{2}} \stackrel{\text { def }}{=} \int_{\partial I} \int_{S}
$$

Remark II.1 The boundary of an oriented geometric object is constituted by its faces endowed with the induced orientation (Figs. 4 and 6). For the case of a time interval $I=\left[T_{1}, T_{2}\right]$, the faces that appear in the boundary $\partial I$ correspond to the two time instants $T_{1}$ and $T_{2}$. If the time interval $I$ is internally oriented in the direction of increasing time, $T_{1}$ appears in $\partial I$ oriented as a source, whereas $T_{2}$ appears in it oriented as a sink. However, as time instants, $T_{1}$ and $T_{2}$ are endowed with a default orientation of their own. Let us assume that the default internal orientation of all time instants is as sinks; it follows that $\partial I$ is constituted by $T_{2}$ taken with its default orientation and by $T_{1}$ taken with the opposite of its default orientation. We can express this fact symbolically, writing $\partial I=$ $T_{2}-T_{1}$, where the "minus" sign signals the inversion of the orientation of $T_{1}$. Correspondingly, if there is a quantity $Q$ associated with the time instants, and $Q_{1}$ and $Q_{2}$ are associated with $T_{1}$ and $T_{2}$, respectively, the quantity $Q_{2}-Q_{1}$ will be associated with $\partial I$. We will give these facts a more precise formulation later, using the concepts of chain and cochain. For now, this example gives a first idea of the key role played by the concept of orientation of space-time geometric objects, in a number of common mathematical operations such as the increment of a quantity and the fact that an expression like $\int_{T_{1}}^{T_{2}} d f$ corresponds to $\left(\left.f\right|_{T_{2}}-\left.f\right|_{T_{1}}\right)$ and not to its opposite. In this context, we alert the reader to the fact that if the time axis is externally oriented, it is the time instants that are oriented by means of a (through) direction, whereas the time instants themselves are oriented as sources or sinks. 
With these definitions [Eqs. (31) and (32)], Eqs. (23) through (29) become

$$
\begin{aligned}
\int_{T} \int_{\partial V} \mathbf{B} & =\int_{T} \int_{V} 0 \\
\int_{I} \int_{\partial S} \mathbf{E}+\int_{\partial I} \int_{S} \mathbf{B} & =\int_{I} \int_{S} \mathbf{0} \\
\int_{\tilde{I}} \int_{\partial \tilde{V}} \mathbf{J}+\int_{\partial \tilde{I}} \int_{\tilde{V}} \rho & =\int_{\tilde{I}} \int_{\tilde{V}} 0 \\
\int_{T} \int_{\partial S} \mathbf{A} & =\int_{T} \int_{S} \mathbf{B} \\
-\int_{I} \int_{\partial L} V-\int_{\partial I} \int_{L} \mathbf{A} & =\int_{I} \int_{L} \mathbf{E} \\
\int_{\tilde{I}} \int_{\partial \tilde{V}} \mathbf{D} & =\int_{\tilde{T}} \int_{\tilde{V}} \rho \\
\int_{\tilde{I}} \int_{\partial \tilde{S}} \mathbf{H}-\int_{\partial \tilde{I}} \int_{\tilde{S}} \mathbf{D} & =\int_{\tilde{I}} \int_{\tilde{S}} \mathbf{J}
\end{aligned}
$$

The equations in this form can be used to determine the correct association of physical quantities with geometric objects.

\section{Classification of Physical Quantities}

In Eqs. (33) through (39), we can identify a number of recurrent terms and deduce from them an association of physical quantities with geometric objects. From Eqs. (33) and (34) we get

$$
\begin{aligned}
& \int_{I} \int_{L} \mathbf{E} \Rightarrow(L \times I) \\
& \int_{T} \int_{S} \mathbf{B} \Rightarrow(S \times T)
\end{aligned}
$$

where the arrow means "is associated with." The term in Eq. (4I) confirms the association of magnetic induction with surfaces and suggests a further one with time instants, whereas Eq. (40) shows that the electric field is associated with lines and time intervals. These geometric objects are endowed with internal orientation, as follows from the analysis made previously for the orientational issues in Faraday's law. 
The status of electric current and charge as a physical quantity can be deduced from Eq. (35), which gives the terms

$$
\begin{gathered}
\int_{\tilde{I}} \int_{\tilde{S}} \mathbf{J} \Rightarrow(\tilde{S} \times \tilde{I}) \\
\int_{\tilde{T}} \int_{\tilde{V}} \rho \Rightarrow(\tilde{V} \times \tilde{T})
\end{gathered}
$$

which show that electric current is associated with surfaces and time intervals, whereas charge is associated with volumes and time instants. Since the current is due to a flow of charges through the surface, a natural external orientation for surfaces follows. Given this association of electric current with externally oriented surfaces, the volumes to which charge content is associated must also be externally oriented to permit direct comparison of the sign of the quantities in Eq. (35).

The same rationale can be applied to the terms appearing in Eqs. (38) and (39); that is,

$$
\begin{gathered}
\int_{\tilde{I}} \int_{\tilde{L}} \mathbf{H} \Rightarrow(\tilde{L} \times \tilde{I}) \\
\int_{\tilde{T}} \int_{\tilde{S}} \mathbf{D} \Rightarrow(\tilde{S} \times \tilde{T})
\end{gathered}
$$

This shows that the magnetic field is associated with lines and time intervals and the electric displacement with surfaces and time instants. As for orientation, the magnetic field is traditionally associated with internally oriented lines but this choice requires the right-hand rule to make the comparison, in Eq. (39), of the direction of $\mathbf{H}$ along $\partial \tilde{S}$ with the direction of the current flow through the surface $\tilde{S}$. Hence, so that the use of the right-hand rule can be dispensed with, the magnetic field must be associated with externally oriented lines. The same argument applies in suggesting an external orientation for surfaces to which electric displacement is associated.

Finally, Eqs. (36) and (37) give the terms

$$
\begin{aligned}
& \int_{I} \int_{P} V \Rightarrow(P \times I) \\
& \int_{T} \int_{L} \mathbf{A} \Rightarrow(L \times T)
\end{aligned}
$$

which show that the scalar potential is associated with points and time intervals, whereas the vector potential is associated with lines and time instants. From the association of the electric field with internally oriented lines, it follows that for the electromagnetic potentials, the orientation is also internal. 


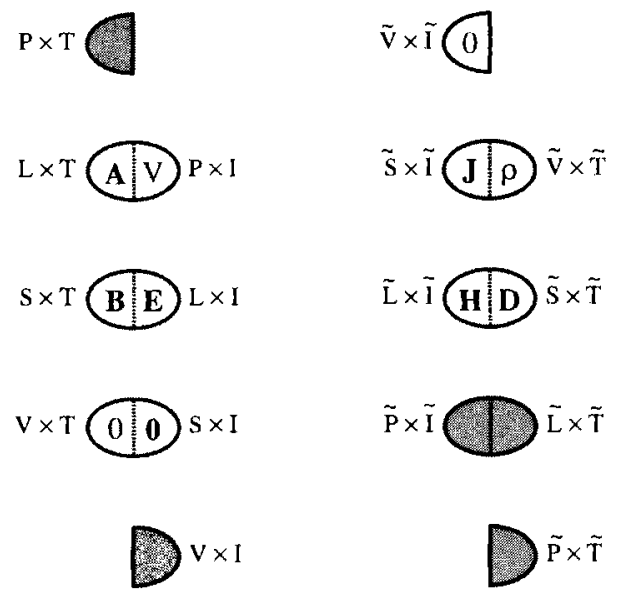

FIgURE 9. The Tonti classification diagram of local electromagnetic quantities.

The null right-hand-side terms in Eqs. (33) through (35) remain to be taken into consideration. We will see subsequently that these terms express the vanishing of magnetic flux creation (or the nonexistence of magnetic charge) and the vanishing of electric charge creation, respectively. For now, we will simply insert them as zero terms in the appropriate slot of the classification diagram for the physical quantities of electromagnetism, which summarizes the results of our analysis (Fig. 9).

\section{Space-Time Viewpoint}

The terms $\int_{\tilde{T}} \int_{\tilde{V}} \rho$ and $\int_{\tilde{I}} \int_{\tilde{S}} \mathbf{J}$ in Eqs. (42) and (43) refer to the same global physical quantity: electric charge. Moreover, total integration is performed in both cases on externally oriented, three-dimensional domains in space-time. We can, therefore, say that electric charge is actually associated with externally oriented, three-dimensional space-time domains of which a three-dimensional space volume considered at a time instant, and a three-dimensional space surface considered during a time interval, are particular cases. To distinguish these two embodiments of the charge concept, we use the terms charge content, referring to volumes and time instants, and charge flow, referring to surfaces and time intervals. A similar distinction can be drawn for other quantities. For example, the terms $\int_{I} \int_{L} \mathbf{E}$ and $\int_{T} \int_{S} \mathbf{B}$ in Eqs. (40) and (41) are both magnetic fluxes associated with two-dimensional space-time domains of which we could say that the electric field refers to a "flow" of magnetic flux tubes which cross internally oriented lines, while magnetic induction refers to a surface "content" 
of such tubes. Since the term content refers properly to volumes, and the term flow to surfaces, it appears preferable to distinguish the two manifestations of each global quantity by using an index derived from the letter traditionally used for the corresponding local quantity, as in

$$
\begin{gathered}
\int_{\tilde{T}} \int_{\tilde{V}} \rho=Q^{\rho}(\tilde{V} \times \tilde{T}) \\
\int_{\tilde{I}} \int_{\tilde{S}} \mathbf{J}=Q^{j}(\tilde{S} \times \tilde{I})
\end{gathered}
$$

and

$$
\begin{aligned}
& \int_{T} \int_{S} \mathbf{B}=\phi^{b}(S \times T) \\
& \int_{I} \int_{L} \mathbf{E}=\phi^{e}(L \times I)
\end{aligned}
$$

The same argument can be applied to electric flux,

$$
\begin{aligned}
& \int_{\tilde{T}} \int_{\tilde{S}} \mathbf{D}=\psi^{d}(\tilde{S} \times \tilde{T}) \\
& \int_{\tilde{I}} \int_{\tilde{L}} \mathbf{H}=\psi^{h}(\tilde{L} \times \tilde{I})
\end{aligned}
$$

and to the potentials in global form,

$$
\begin{aligned}
& \int_{T} \int_{L} \mathbf{A}=\mathcal{U}^{a}(L \times T) \\
& \int_{I} \int_{P} V=\mathcal{U}^{v}(P \times I)
\end{aligned}
$$

With these definitions we can fill in the classification diagram of global electromagnetic quantities (Fig. 10).

Note that the classification diagram of Figure (10) emphasizes the pairing of physical quantities which happen to be the static and dynamic manifestations of a unique space-time entity. We can group these variables under a single heading, obtaining a classification diagram of the space-time global electromagnetic quantities $\mathcal{U}, \phi, \psi$, and $Q$ (Fig. 11), which corresponds to the one that could be drawn for local quantities in four-dimensional notation. Note also that all the global quantities of a column possess the same physical dimension; for example, the terms in Eqs. (48), (49), (52), and (53) all have the physical dimension of electric charge. Nonetheless, quantities appearing in different rows of a column refer to different physical quantities since, even 

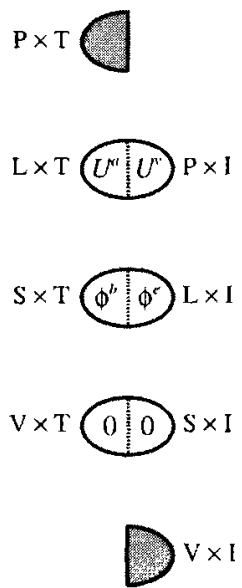<smiles>C1C[Al]C1</smiles>
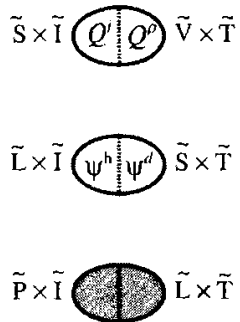

Figure 10. The Tonti classification diagram of global electromagnetic quantities.

if the physical dimension is the same, the underlying space-time oriented geometric object is not. This fact is reflected in the relativistic behavior of these quantities. When an observer changes his or her reference frame, his or her perception of what is time and what is space changes and with it his or her method of splitting a given space-time physical quantity into its two "space plus time" manifestations. Hence, the transformation laws, which account for
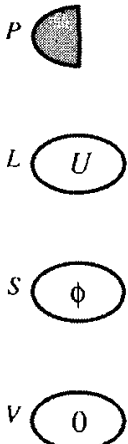

$H$

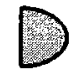

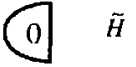
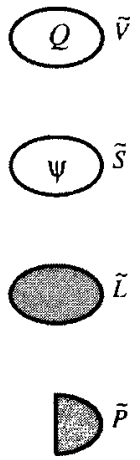

Figure 11. The Tonti classification diagram of global electromagnetic quantities, referring to space-time geometric objects. 
the change of reference frame, will combine only quantities referring to the same space-time oriented object. In a four-dimensional treatment such quantities will be logically grouped within a unique entity (e.g., the charge-current vector; the four-dimensional potentials; the first and second electromagnetic tensor-or the corresponding differential forms-with groupings $\mathbf{E}$ and $\mathbf{B}$, and $\mathbf{H}$ and $\mathbf{D}$, respectively; and so on).

\section{E. Topological Laws}

Now that we have seen how to proceed to the individuation and classification of the physical quantities of a theory, there remains, as a last step in the determination of the structure of the theory itself, the establishment of the links existing between the quantities, accompanied by an analysis of the properties of these links. As anticipated, the main result of this further analysis-valid for all field theories-will be the singling out of a set of physical laws, which lend themselves naturally to a discrete rendering, opposed to another set of relations, which constitute instead an obstacle to the complete discrete rendering of field problems.

It is apparent from the definitions given in Eqs. (48) through (55), that Eqs. (33) through (39) can be rewritten in terms of global quantities only, as follows:

$$
\begin{aligned}
\phi^{b}(\partial V \times T) & =0(V \times T) \\
\phi^{e}(\partial S \times I)+\phi^{b}(S \times \partial I) & =0(S \times I) \\
Q^{j}(\partial \tilde{V} \times \tilde{I})+Q^{\rho}(\tilde{V} \times \partial \tilde{I}) & =0(\tilde{V} \times \tilde{I}) \\
\mathcal{U}^{a}(\partial S \times T) & =\phi^{b}(S \times T) \\
-\mathcal{U}^{v}(\partial L \times I)-\mathcal{U}^{a}(L \times \partial I) & =\phi^{e}(L \times I) \\
\psi^{d}(\partial \tilde{V} \times \tilde{T}) & =Q^{\rho}(\tilde{V} \times \tilde{T}) \\
\psi^{h}(\partial \tilde{S} \times \tilde{I})-\psi^{d}(\tilde{S} \times \partial \tilde{I}) & =Q^{j}(\tilde{S} \times \tilde{I})
\end{aligned}
$$

Note that no material parameters appear in these equations, and that the transition from the local, differential statements in Eqs. (7) through (13) to these global statements was performed without recourse to any approximation. This proves their intrinsic discrete nature. Let us examine and interpret these statements one by one.

Gauss's magnetic law [Eq. (56)] asserts the vanishing of magnetic flux associated with closed surfaces $\partial V$ in space considered at a time instant $T$. From 


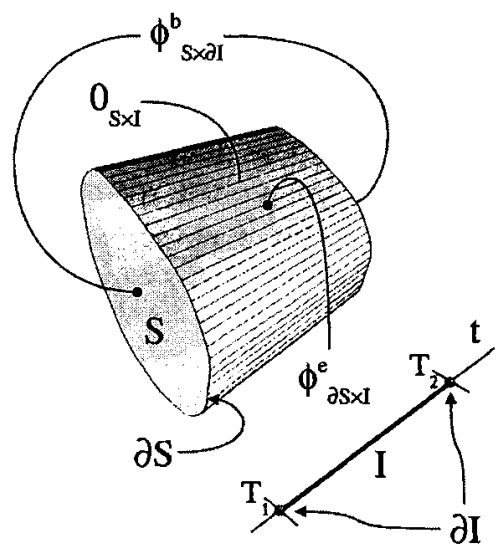

FIGURE 12. Faraday's induction law admits a geometric interpretation as a conservation law on a space-time cylinder. The (internal) orientation of geometric objects is not represented.

what we said previously about space-time objects, there must be a corresponding assertion for timelike closed surfaces. Faraday's induction law [Eq. (57)] is indeed such an assertion for a cylindrical closed surface in space-time constructed as follows (Fig. 12): the surface $S$ at the time instant $T_{1}$ constitutes the first base of a cylinder; the boundary of $S, \partial S$, considered during the time interval $I=\left[T_{1}, T_{2}\right]$, constitutes the lateral surface of the cylinder, which is finally closed by the surface $S$ considered at the time instant $T_{2}$ [remember that $T_{1}$ and $T_{2}$ together constitute the boundary $\partial I$ of the time interval $I$, hence the term $S \times \partial I$ in Eq. (57) represents the two bases of the cylinder] (Bamberg and Sternberg, 1988; Truesdell and Toupin, 1960). This geometric interpretation of Faraday's law is particularly interesting for numerical applications, for it is an exact statement linking physical quantities at times $T<T_{2}$ to a quantity defined at time $T_{2}$. Therefore, this statement is a good starting point for the development of the time-stepping procedure.

In summary, Gauss's law and Faraday's induction law are the space and the space-time parts, respectively, of a single statement: the magnetic flux associated with the boundary of a space-time volume $\mathcal{V}$ is always zero:

$$
\phi(\partial \mathcal{V})=0(\mathcal{V})
$$

(Remember that the boundary of an oriented geometric object must always be thought of as endowed with the induced orientation.) Equation (63), also called the law of conservation of magnetic flux (Truesdell and Toupin, 1960), gives to its right-hand-side term the meaning of a null in the production of magnetic flux. From another point of view, the right-hand side of Eq. (56) expresses 
the nonexistence of magnetic charge and that of Eq. (57) the nonexistence of magnetic charge current.

The other conservation statement of electromagnetism is the law of conservation of electric charge [Eq. (58)]. In strict analogy with the geometric interpretation of Faraday's law, a cylindrical, space-time, closed hypersurface is constructed as follows: the volume $\tilde{V}$ at the time instant $\tilde{T}_{1}$ constitutes the first base of a hypercylinder; the boundary of $\tilde{V}, \partial \tilde{V}$, considered during the time interval $\tilde{I}=\left[\tilde{T}_{1}, \tilde{T}_{2}\right]$, constitutes the lateral surface of the hypercylinder, which is finally closed by the volume $\tilde{V}$ considered at the time instant $\tilde{T}_{2}$. The law of charge conservation asserts the vanishing of the electric charge associated with this closed hypercylinder. This conservation statement can be referred to the boundary of a generic space-time hypervolume $\tilde{\mathcal{H}}$, which yields the following statement, analogous to Eq. (63):

$$
Q(\partial \tilde{\mathcal{H}})=0(\tilde{\mathcal{H}})
$$

In Eq. (64) the zero on the right-hand side states the vanishing of the production of electric charge. Note that in this case a purely spatial statement, corresponding to Gauss's law of magnetostatics [Eq. (56)] is not given, for in four-dimensional space-time a hypervolume can be obtained only as a product of a volume in space multiplied by a time interval.

The two conservation statements [Eqs. (63) and (64)] can be considered the two cornerstones of electromagnetic theory (Truesdell and Toupin, 1960). de Rham (1931) proved that from the global validity of statements of this kind [or, if you prefer, of Eqs. (33) through (35)] in a homologically trivial space follows the existence of field quantities that can be considered the potentials of the densities of the physical quantities appearing in the global statements. In our case we know that the field quantities $V$ and $\mathbf{A}$, defined by Eqs. (10) and (11), are indeed traditionally called the electromagnetic potentials. Correspondingly, the field quantities $\mathbf{H}$ and $\mathbf{D}$ defined by Eqs. (12) and (13) are also potentials and can be called the charge-current potentials (Truesdell and Toupin, 1960). In fact the definition of $\mathbf{H}$ and $\mathbf{D}$ is a consequence of charge conservation, exactly as the definition of $V$ and $\mathbf{A}$ is a consequence of magnetic flux conservation; therefore, neither is uniquely defined by the conservation laws of electromagnetism. Only the choice of a gauge for the electromagnetic potentials and the hypothesis about the media properties for charge-current potentials removes this nonuniqueness.

In any case, the global renditions [Eqs. (59) through (62)] of the equations defining the potentials prove the intrinsic discrete status of Gauss's law of electrostatics, of Maxwell-Ampère's law, and of the defining equations of the electromagnetic potentials. A geometric interpretation can be given to these laws, too. Gauss's law of electrostatics asserts the balance of the electric charge contained in a volume with the electric flux through the surface that bounds 


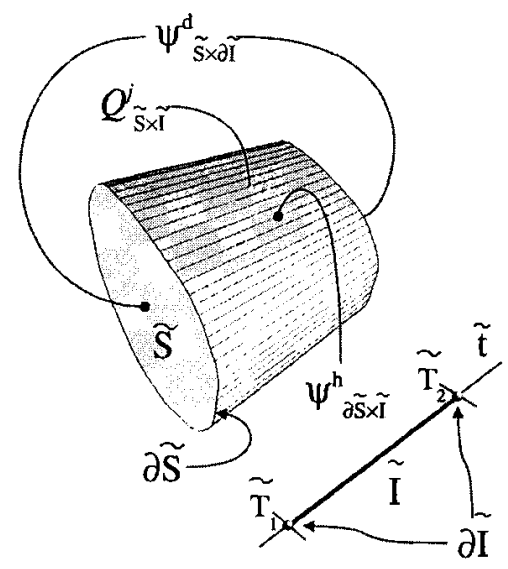

Figure 13. Maxwell-Ampère's law admits a geometric interpretation as a balance law on a space-time cylinder. The (external) orientation of geometric objects is not represented.

the volume. Similarly, Maxwell-Ampère's law defines this balance between the charge contained within a space-time volume and the electric flux through its boundary, which is a cylindrical space-time closed surface analogous to the one appearing in Faraday's law, but with external orientation (Fig. 13). This geometric interpretation, like that of Faraday's law, is instrumental for a correct setup of the time stepping within a numerical procedure.

Equations (61) and (62) can be condensed into a single space-time statement that asserts the balance of the electric charge associated with arbitrary spacetime volumes with the electric flux associated with their boundaries:

$$
\psi(\partial \tilde{\mathcal{V}})=Q(\tilde{\mathcal{V}})
$$

Analogous interpretations hold for Eqs. (59) and (60), relative to a balance of magnetic fluxes associated with space-time surfaces and their boundaries:

$$
\mathcal{U}(\partial \mathcal{S})=\phi(\mathcal{S})
$$

We can insert the global space-time statements [Eqs. (63) through (66)] in the space-time classification diagram of the electromagnetic physical quantities (Fig. 14). Note that all these statements appear as vertical links. These links relate a quantity associated with an oriented geometric object with a quantity associated with the boundary of that object (which has, therefore, the same kind of orientation). What is shown here for the case of electromagnetism applies to the great majority of physical field theories. Typically, a subset of the equations which form a physical field theory link a global quantity associated with an oriented geometric object to the global quantity that, within 

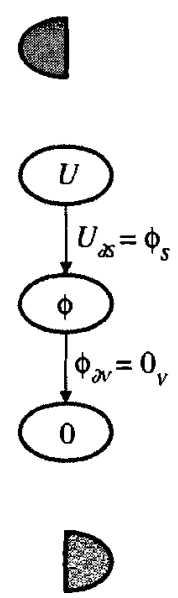
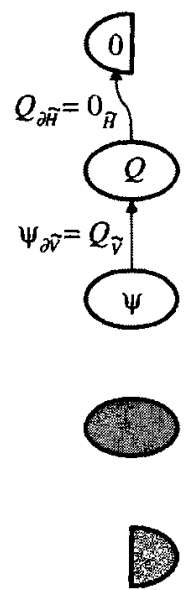

FIGURE 14. The position of topological laws in the Tonti classification diagram of electromagnetic quantities.

the theory, is associated with the boundary of that object (Tonti, 1975). These laws are intrinsically discrete, for they state a balance of these global quantities (or a conservation of them, if one of the terms is zero) whose validity does not depend on metrical or material properties, and is, therefore, invariant for very general transformations. This gives them a "topological significance" (Truesdell and Toupin, 1960), which justifies our calling them topological laws. The significance of this finding for numerical methods is obvious: once the domain of a field problem has been suitably discretized, topological laws can be written directly and exactly in discrete form.

\section{F. Constitutive Relations}

To complete our analysis of the equations of electromagnetism, we must consider the set of constitutive equations, represented, for example, by Eqs. (14) through (16). We emphasize once again that each instance of this kind of equation is only a particular case of the various forms that the constitutive links between the problem's quantities can take. In fact, while topological laws can be considered universal laws linking the field quantities of a theory, constitutive relations are merely definitions of ideal materials given within the framework of that particular field theory (Truesdell and Noll, 1965). In other words, they are abstractions inspired by the observation of the behavior of actual materials. More sophisticated models have terms that account for a wider range of observed material behaviors, such as nonlinearity, anisotropy, nonlocality, 


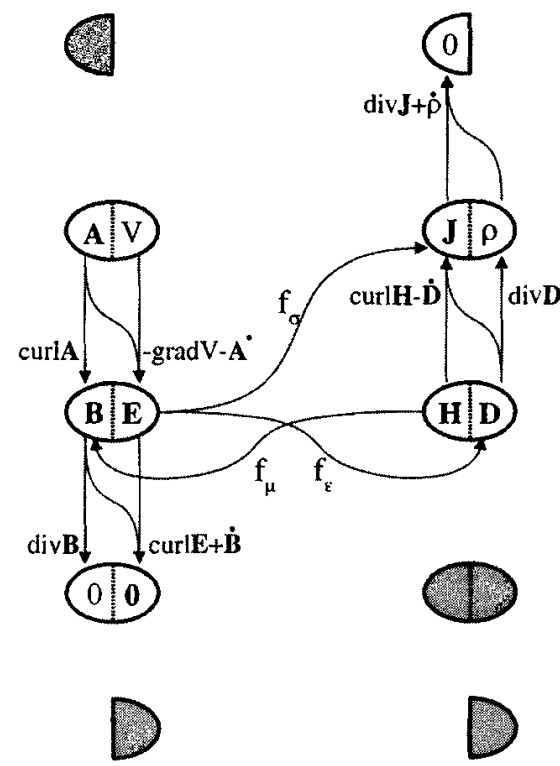

FIGURE 15. The Tonti factorization diagram of electromagnetism in local form. Topological laws are represented by vertical links within columns, whereas constitutive relations are represented by transverse links bridging the two columns of the diagram.

hysteresis, and the combinations thereof (Post, 1997). This added complexity implies usually a greater sophistication of the numerical solvers, but does not change the essence of what we are about to say concerning the discretization of constitutive relations.

If we consider the position of constitutive relations in the classification diagram of the physical quantities of electromagnetism, we observe that they constitute a link that connects the two columns (Fig. 15). This fact reveals that, unlike topological laws, constitutive relations link quantities associated with geometric objects endowed with different kinds of orientation. From the point of view of numerical methods, the main differences with topological laws are the observation that constitutive relations contain material parameters* and the fact that they are not intrinsically discrete. The presence of a term of this kind in the field equations is not surprising, since otherwise-given the intrinsic

${ }^{*}$ In some cases material parameters seemingly disappear from constitutive equations. This is the case, for example, with electromagnetic equations in empty space when we adopt Gaussian units and set $c=1$. This induces the temptation to identify physical quantities - in this case $\mathbf{E}$ and $\mathbf{D}$, and $\mathbf{B}$ and $\mathbf{H}$. respectively. However, the approach based on the association with oriented geometric objects reveals that these quantities have a distinct nature. 
discreteness of topological laws-it would always be possible to exactly discretize and solve numerically a field problem, and we know that this is not the case. Constitutive relations can be transformed into exact links between global quantities only if the local properties do not vary in the domain where the link must be valid. This means that we must impose a series of uniformity requirements on material and field properties for a global statement to hold true. On the contrary, since, aside from discontinuities, these requirements are automatically satisfied in the small, the local statement always applies. The uniformity requirement is in fact the method used to experimentally investigate these laws. For example, we can investigate the constitutive relation

$$
\mathbf{D}=\varepsilon \mathbf{E}
$$

examining a capacitor with two planar parallel plates of area $A$, having a distance $l$ between them and filled with a uniform, linear, isotropic medium having relative permittivity $\varepsilon_{r}$. With this assumption, Eq. (67) corresponds approximately to

$$
\frac{\psi}{A}=\varepsilon \frac{V}{l}
$$

where $\psi$ is the electric flux and $V$ the voltage between the plates. Note that to write Eq. (68), besides using the material parameter $\varepsilon$, we invoke the concepts of planarity, parallelism, area, distance, and orthogonality, which are not topological concepts. This shows that, unlike topological laws, constitutive relations imply the recourse to metrical concepts. This is not apparent in Eq. (67), for-as explained previously-the use of vectors to represent field quantities tends to hide the geometric details of the theory. Equation (67) written in terms of differential forms, or a geometric representation thereof, reveals the presence, within the link, of the metric tensor (Burke, 1985; Post, 1997). The local nature of constitutive relations can be interpreted by saying that these equations summarize at a macroscopic level something going on at a subjacent scale. This hypothesis may help the intuition, but it is not necessary if we are willing to interpret them as definitions of ideal materials. By so doing, we can avoid the difficulties implicit in the creation of a convincing derivation of field concepts from a corpuscular viewpoint.

There is other information about constitutive equations that can be derived by observing their position in the factorization diagram. These are not of direct relevance from a numerical viewpoint but can help us to understand better the nature of each term. For example, it has been observed that when the two columns of the factorization diagram are properly aligned according to duality, constitutive relations linked to irreversible processes (e.g., Ohm's law linking $\mathbf{E}$ and $\mathbf{J}$ in Fig. 15) appear as slanted links, whereas those representing reversible processes appear as horizontal links (Tonti, 1975). 


\section{Constitutive Equations and Discretization Error}

We anticipated in the preceding discussion that, from our point of view, the main consequence of the peculiar nature of constitutive relations lies in their preventing, in general, the attainment of an exact discrete solution. By "exact discrete solution," we mean the exact solution of the continuous mathematical model (e.g., a partial differential equation) into which the physical problem is usually transformed. We hinted in the Introduction at the fact that the numerical solution of a field problem implies three phases (Fig. 1):

1. The transformation of the physical problem into a mathematical model

2. The discretization of the mathematical model

3 . The solution of the system of algebraic equations produced by the discretization

(The fourth phase represented in Fig. 1, the approximate reconstruction of the field function based on the discrete solution, obviously does not affect the accuracy of the discrete solution.) Correspondingly, there will be three kinds of errors (Fig. 16; Ferziger and Perić, 1996; Lilek and Perić, 1995):

1. The modeling error

2. The discretization error

3. The solver error

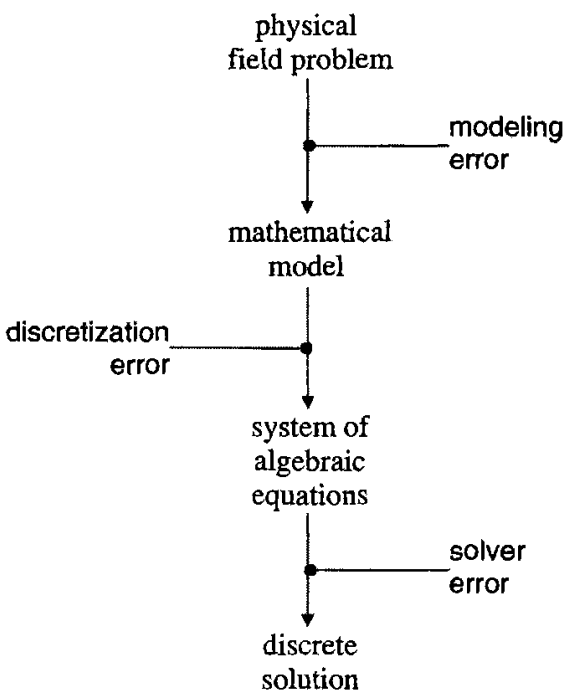

FIGURE 16. The three kinds of errors associated with the numerical solution of a field problem. 
Modeling errors are a consequence of the assumptions about the phenomena and processes, made during the transition from the physical problem to its mathematical model in terms of equations and boundary conditions. Solver errors are a consequence of the limited numerical precision and time available for the solution of the system of algebraic equations. Discretization errors act between these two steps, preventing the attainment of the exact discrete solution of the mathematical model, even in the hypothesis that our algebraic solvers were perfect.

The existence of discretization errors is a well-known fact, but it is the analysis based on the mathematical structure of physical theories that reveals where the discretization obstacle lies; that is, within constitutive relations, topological laws not implying in themselves any discretization error. As anticipated in the Introduction, this in turn suggests the adoption of a discretization strategy in which what is intrinsically discrete is included as such in the model, and the discretization effort is focused on what remains. It must be said, however, that once the discretization error is brought into by the presence of the constitutive terms, it is the joint contribution of the approximation implied by the discretization of these terms and of our enforcing only a finite number of topological relations in place of the infinitely many that are implied by the corresponding physical law that shapes the actual discretization error. This fact will be examined in detail subsequently.

\section{G. Boundary Conditions and Sources}

A field problem includes, in addition to the field equations, a set of boundary conditions and the specification that certain terms appearing in the equations are assigned as sources. Boundary conditions and sources are a means to limit the scope of the problem actually analyzed, for they summarize the effects of interactions with domains or phenomena that we choose not to consider in detail. Let us see how boundary conditions and sources enter into the framework developed in the preceding sections for the equations, with a classification that parallels the distinction between topological laws and constitutive relations.

When boundary conditions and sources are specified as given values of some of the field quantities of the problem, they correspond in our scheme to global values assigned to some geometric object placed along the boundary or lying within the domain. Hence, the corresponding values enter the calculations exactly, but for the possibly limited precision with which they are calculated from the corresponding field functions (usually by numerical integration) when they are not directly given as global quantities. Consequently, in this case these terms can be assimilated with topological prescriptions. 
In other cases boundary and source terms are assigned in the form of equations linking a problem's field variable to a given excitation. In these cases, these terms must be considered as additional constitutive relations to which all the considerations made previously for this kind of equation apply. In particular, within a numerical formulation, such terms must be subjected to a specific discretization process. For example, this is the case for convective boundary conditions in heat transfer problems.

In still other cases boundary conditions summarize the effects on the problem domain of the structure of that part of space-time which lies outside the problem domain. Think, for example, about radiative boundary conditions in electrodynamics, and inlet and outlet boundary conditions in fluid dynamics. In these cases, one cannot give general prescriptions, for the representation depends on the geometric and physical structure of this "outside." Physically speaking, a good approach consists of extending the problem's domain, enclosing it in a (thin) shell whose properties account, with a sufficient approximation, for the effect of the whole space surrounding the domain, and whose boundary conditions belong to one of the previous kinds. This shell can then be modeled and discretized by following the rules used for the rest of the problem's domain. However, devising the properties of such a shell is usually not a trivial task. In any case, the point is that boundary conditions and source terms can be brought back to topological laws and constitutive relations by physical reasoning, and from there they require no special treatment with respect to what applies to these two categories of relations.

\section{H. The Scope of the Structural Approach}

The example of electromagnetism, examined in detail in the previous sections, shows that to approach the numerical solution of a field problem by taking into account its mathematical structure, we must first classify the physical quantities appearing in the field equations, according to their association with oriented geometric objects, and then factorize the field equations themselves to the point of being able to draw the factorization diagram for the field theory to which the problem belongs. The result will be a distinction of topological laws, which are intrinsically discrete, from constitutive relations, which admit only approximate discrete renderings (Fig. 17).

Let us examine briefly how this process works for other theories and the difficulties we can expect to encounter. From electromagnetism we can easily derive the diagrams of electrostatics and magnetostatics. If we drop the time dependence, the factorization diagram for electromagnetism splits naturally into the two distinct diagrams of electrostatics and magnetostatics (Figs. 18 and 19). 


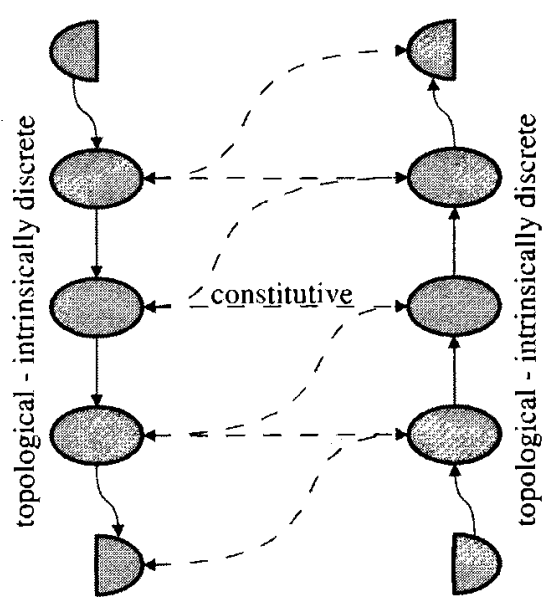

FIGURE 17. The distinction between topological and constitutive terms of the field equations, as it appears in the Tonti factorization diagram. Topological laws appear as vertical links and are intrinsically discrete, whereas constitutive relations appear as transverse links and in general permit only approximate discrete renderings.

Given the well-known analogy between stationary heat conduction and electrostatics (Burnett, 1987; Maxwell, 1884), one would expect to derive the diagram for this last theory directly from that of electrostatics. An analysis of physical quantities reveals, however, that the analogy is not perfect. Temperature, which is linked by the analogy to electrostatic potential $V$, is indeed associated, like $V$, to internally oriented points and time intervals, but heat flow density, traditionally considered analogous with electric displacement $\mathbf{D}$, is in fact associated with externally oriented surfaces and time intervals, whereas $\mathbf{D}$ is associated with surfaces and time instants. In the stationary case, this distinction makes little difference, but we will see later, in Fig. 20, that this results in a slanting of the constitutive link between the temperature gradient $\mathbf{g}$ and the diffusive heat flux density $\mathbf{q}_{d}$, whereas the constitutive link between $\mathbf{E}$ and $\mathbf{D}$ is not slanted. This reflects the irreversible nature of the former process, as opposed to the reversible nature of the latter.

Since the heat transfer equation can be considered a prototype of all scalar transport equations, it is worth examining in detail, including both the nonstationary and the convective terms. A heat transfer equation that is general enough for our purposes can be written as follows (Versteeg and Malalasekera, 1995):

$$
\frac{\partial(\rho c \theta)}{\partial t}+\operatorname{div}(\rho c \theta \mathbf{u})-\operatorname{div}(k \operatorname{grad} \theta)=\sigma
$$




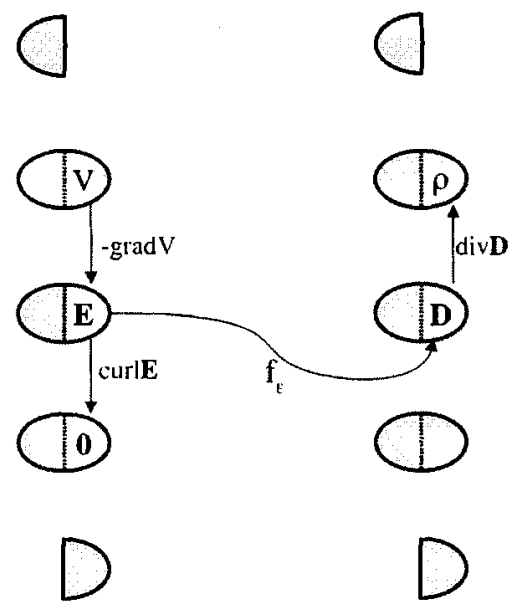

FIGURE 18. The Tonti factorization diagram for electrostatics in local form.

where $\theta$ is the temperature, $\rho$ is the mass density, $c$ is the specific heat, $\mathbf{u}$ is the fluid velocity, $k$ is the thermal conductivity, and $\sigma$ is the heat production density rate. Note that we always start with field equations written in local form, for these equations usually include constitutive terms. We must first factor out these terms before we can write the topological terms in their primitive, discrete form. Disentangling the constitutive relations from the topological laws, we

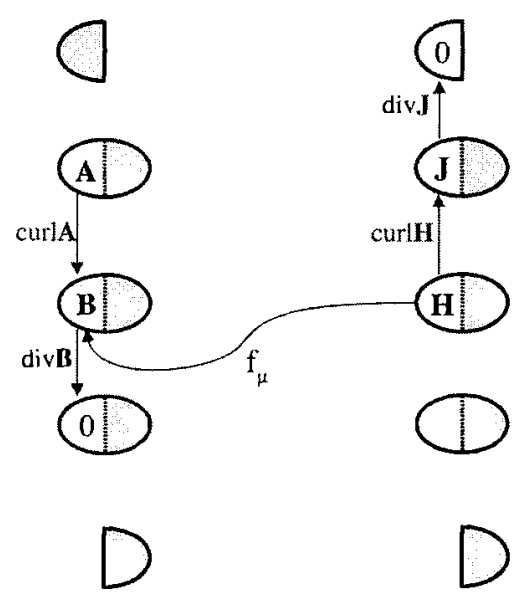

FIGURE 19. The Tonti factorization diagram for magnetostatics in local form. 
obtain the following set of topological equations,

$$
\begin{aligned}
\operatorname{grad} \theta & =\mathbf{g} \\
\frac{\partial q_{c}}{\partial t}+\operatorname{div} \mathbf{q}_{u}+\operatorname{div} \mathbf{q}_{d} & =\sigma
\end{aligned}
$$

and the following set of constitutive equations,

$$
\begin{aligned}
\mathbf{q}_{u} & =\rho c \theta \mathbf{u} \\
\mathbf{q}_{d} & =-k \mathbf{g} \\
q_{c} & =\rho c \theta
\end{aligned}
$$

To write Eqs. (70) through (74), we have introduced four new local physical quantities: the temperature gradient $\mathbf{g}$, the diffusive heat flow density $\mathbf{q}_{d}$, the convective heat flow density $\mathbf{q}_{u}$, and the heat content density $q_{c}$. Note that of the three constitutive equations, Eq. (72) appears as a result of a driving source term, with the parameter $\mathbf{u}$ derived from an "external" problem. This is an example of how the information about interacting phenomena is carried by terms appearing in the form of constitutive relations. Another example is given by boundary conditions describing a convective heat exchange through a part $\partial D_{v}$ of the domain boundary. If $\theta_{\infty}$ is the external ambient temperature, $h$ is the coefficient of convective heat exchange, and we denote with $\mathbf{q}_{v}$ and $\theta_{v}$ the convective heat flow density and the temperature at a generic point of $\partial D_{v}$, we can write

$$
\mathbf{q}_{v}=h\left(\theta_{v}-\theta_{\infty}\right)
$$

An alternative approach is to consider this as an example of coupled problems, where the phenomena that originate the external driving terms are treated as separate interacting problems, which must also be discretized and solved. In this case, a factorization diagram must be built for each physical field problem intervening in the whole problem, and what is treated here as driving terms become links between the diagrams. In these cases, a preliminary classification of all the physical variables appearing in the different phenomena is required, so that we can select the best common discretization substratum, especially for what concerns the geometry.

Putting the topological laws, with the new boundary term [Eq. (75)], in full integral form, we have

$$
\begin{aligned}
\int_{I} \int_{\partial L} \theta & =\int_{I} \int_{L} \mathbf{g} \\
\int_{\tilde{I}} \int_{\partial \tilde{V}} \mathbf{q}_{v}+\int_{\tilde{I}} \int_{\partial \tilde{V}} \mathbf{q}_{u}+\int_{\tilde{I}} \int_{\partial \tilde{V}} \mathbf{q}_{d}+\int_{\partial \tilde{I}} \int_{\tilde{V}} q_{c} & =\int_{\tilde{I}} \int_{\tilde{V}} \sigma
\end{aligned}
$$


We can define the following global quantities

$$
\begin{aligned}
& \int_{I} \int_{P} \theta=\Theta(P \times I) \\
& \int_{I} \int_{L} \mathbf{g}=G(L \times I) \\
& \int_{\tilde{I}} \int_{\tilde{S}} \mathbf{q}_{u}=Q_{u}(\tilde{S} \times \tilde{I}) \\
& \int_{\tilde{I}} \int_{\tilde{S}} \mathbf{q}_{v}=Q_{v}(\tilde{S} \times \tilde{I}) \\
& \int_{\tilde{I}} \int_{\tilde{S}} \mathbf{q}_{d}=Q_{d}(\tilde{S} \times \tilde{I}) \\
& \int_{\tilde{T}} \int_{\tilde{V}} q_{c}=Q_{c}(\tilde{V} \times \tilde{T}) \\
& \int_{\tilde{I}} \int_{\tilde{V}} \sigma=F(\tilde{V} \times \tilde{I})
\end{aligned}
$$

with the temperature impulse $\Theta$ associated with internally oriented points and time intervals; the thermal tension $G$ associated with internally oriented lines and time intervals; the convective and diffusive heat flows $Q_{u}, Q_{v}$, and $Q_{d}$ associated with externally oriented surfaces and time intervals; the heat content $Q_{c}$ associated with externally oriented volumes and time instants; and the heat production $F$ associated with externally oriented volumes and time intervals. The same associations hold for the corresponding local quantities. This permits us to write Eqs. (76) and (77) in terms of global quantities only:

$$
\begin{aligned}
\Theta(\partial L \times I) & =G(L \times I) \\
Q_{v}(\partial \tilde{V} \times I)+Q_{u}(\partial \tilde{V} \times I)+Q_{d}(\partial \tilde{V} \times I)+Q_{c}(\tilde{V} \times \partial \tilde{I}) & =F(\tilde{V} \times \tilde{I})
\end{aligned}
$$

Note that Eq. (86) is the natural candidate for the setup of a time-stepping scheme within a numerical procedure, for it links exactly quantities defined at times which precede the final instant of the interval $I$ to the heat content $Q_{c}$ at the final instant. This completes our analysis of the structure of heat transfer problems represented by Eq. (69) and establishes the basis for their discretization. The corresponding factorization diagram in terms of local field quantities is depicted in Fig. 20.

Along similar lines one can conduct the analysis for many other theories. No difficulties are to be expected for those that happen to be characterizedlike electromagnetism and heat transfer-by scalar global quantities. More complex are cases of theories in which the global quantities associated with 

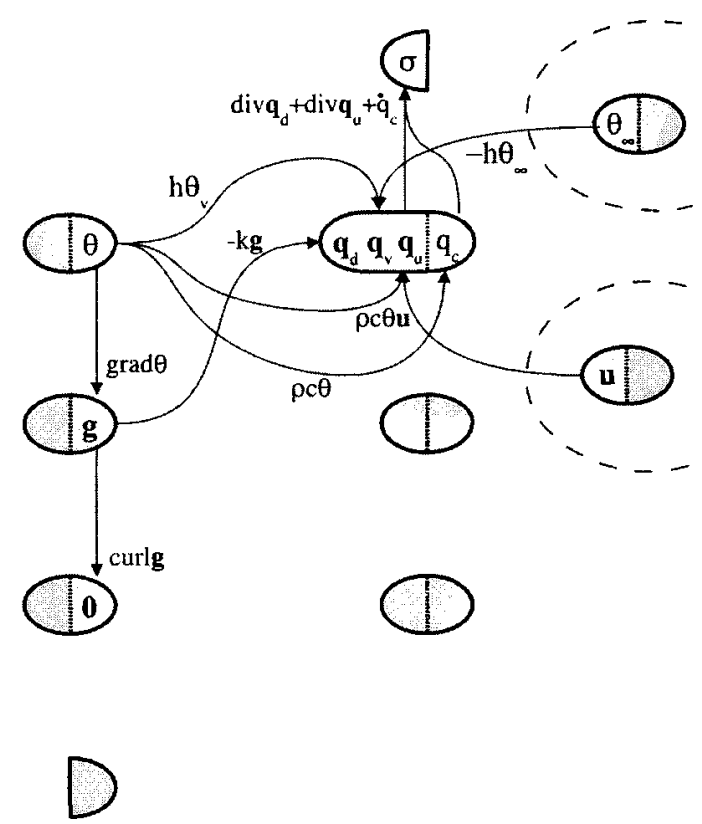

Figure 20. The Tonti factorization diagram for the heat transfer equation in local form. Note the presence of terms derived from the diagrams of other theories or other domains.

geometric objects are vectors or more complex mathematical entities. This is the case of fluid dynamics and continuum mechanics (in which vector quantities such as displacements, velocities, and forces are associated with geometric objects). In this case, the deduction of the factorization diagram can be a difficult task, for one must first tackle a nontrivial classification task for quantities that have, in local form, a tensorial nature, and then disentangle the constitutive and topological factors of the corresponding equations. Moreover, for vector theories it is more difficult to pass silently over the fact that to compare or add quantities defined at different space-time locations (even scalar quantities, in fact), we need actually a connection defined in the domain.

To simplify things, one could be tempted to write the equations of fluid dynamics as a collection of scalar transport equations, hiding within the source term everything that does not fit in an equation of the form of Eq. (69), and to apply to these equations the results of the analysis of the scalar transport equation. However, it is clear that this approach prevents the correct association of physical quantities with geometric objects and is, therefore, far from the spirit advocated in this work. Moreover, the inclusion of too many interaction terms within the source terms can spoil the significance of the analysis, for example, 
hiding essential nonlinearities. ${ }^{*}$ Finally, it must be said that, given a field problem, one could consider the possibility of adopting a Lagrangian viewpoint in place of the Eulerian one that we have considered so far. The approach presented here applies, strictly speaking, only to a Eulerian approach. Nevertheless, the benefits derived from a proper association of physical quantities to oriented geometric objects extend also to a Lagrangian approach. Moreover, the case of moving meshes is included without difficulties in the space-time discretization described subsequently, and in particular in the reference discretization strategy that will be introduced in the section on numerical methods (Section IV).

\section{REPRESENTATIONS}

We have analyzed the structure of field problems, aiming at their discretization. Our final goal is the actual derivation of a class of discretization strategies that comply with that structure. To this end, we must first ascertain what has to be modeled in discrete terms. A field problem includes the specification of a space-time domain and of the physical phenomena that are to be studied within it. The representation of the domain requires the development of a geometric model to which mathematical models of physical quantities and material properties must be linked, so that physical laws can finally be modeled as relations between these entities. Hence, our first task must be the development of a discrete mathematical model for the domain geometry. This will be subsequently used as a support for a discrete representation of fields, complying with the principles derived from the analysis of the mathematical structure of physical theories. The discrete representation of topological laws, then, follows naturally and univocally. This is not the case for constitutive relations, for the discretization of which various options exist. In the next sections we will examine a number of discrete mathematical concepts that can be used in the various discretization steps.

\section{A. Geometry}

The result of the discretization process is the reduction of the mathematical model of a problem having an infinite number of degrees of freedom into one with a finite number. This means that we must find a finite number of entities

\footnotetext{
*As quoted by Moore (1989), Schrödinger, in a letter to Born, wrote: " 'If everything were linear, nothing would influence nothing,' said Einstein once to me. That is actually so. The champions of linearity must allow zero-order terms, like the right side of the Poisson equation, $\Delta V=-4 \pi \rho$. Einstein likes to call these zero-order terms 'asylum ignorantiae"” (p. 381).
} 
which are related in a known way to the physical quantities of interest. If we focus our attention on the fields, and think in terms of the usual continuous representations in terms of scalar or vector functions, the first thing that comes to mind is the plain sampling of the field functions at a finite number of pointsusually called nodes - within the domain. This sampling produces a collection of nodal scalar or vector values, which eventually appear in the system of algebraic equations produced by the discretization. Our previous analysis reveals, however, that this nodal sampling of local field quantities is unsuitable for a discretization which aims at preserving the mathematical structure of the field problem, since such a discretization requires the association of global physical quantities with geometric objects that are not necessarily points. From this point of view, a sound discretization of geometry must provide all the kinds of oriented geometric objects that are actually required to support the global physical quantities appearing within the problem, or at least, those appearing in its final formulation as a set of algebraic equations. Let us see how this reflects on mesh properties.

\section{Cell Complexes}

Our meshes must allow the housing of global physical quantities. Hence, their basic building blocks must be oriented geometric objects. Since we are going to make heavy use of concepts belonging to the branch of mathematics called algebraic topology, we will adopt the corresponding terminology. Algebraic topology is a branch of mathematics that studies the topological properties of spaces by associating them with suitable algebraic structures, the study of which gives information about the topological structure of the original space (Hocking and Young, 1988). In the first stages of its development, this discipline considered mostly spaces topologically equivalent to polytopes (polygons, polyhedra, etc.). Many results of algebraic topology are obtained by considering the subdivisions in collections of simple subspaces, of the spaces under scrutiny. Understandably, then, many concepts used within the present work were formalized in that context. In the later developments of algebraic topology, much of the theory was extended from polytopes to arbitrary compact spaces. The concepts involved became necessarily more abstract, and the recourse to simple geometric constructions waned. Since all our domains are assumed to be topologically equivalent to polytopes, we need and will refer only to the ideas and methods of the first, more intuitive version of algebraic topology.

With the new terminology, what we have so far called an oriented $p$-dimensional geometric object will be called an oriented p-dimensional cell, or simply a $p$-cell, since all cells will be assumed to be oriented, even if this is not explicitly stated. From the point of view of algebraic topology, a $p$-cell $\tau_{p}$ in a domain $D$ can be defined simply as a set of points that is homeomorphic to a closed $p$-ball $B_{p}=\left\{\mathbf{x} \in \mathbb{R}^{p}:\|\mathbf{x}\| \leq 1\right\}$ of the Euclidean $p$-dimensional 

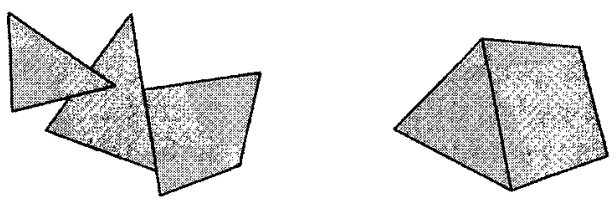

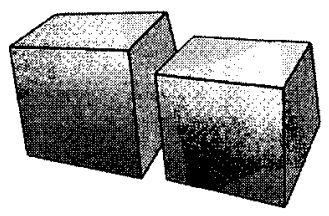

(a)

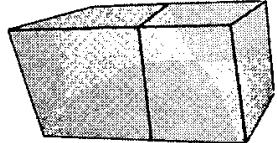

(b)

FIGURE 21. (a) Improper and (b) proper joining of cells.

space (Franz, 1968; Hocking and Young, 1988; Whitney, 1957). To model our domains as generic topological spaces, however, would be entirely too generic. We can assume, without loss of generality, that the domain $D$ of our problem is an $n$-dimensional differentiable manifold of which our $p$-cells are $p$-dimensional regular subdomains* (Boothby, 1986). With these hypotheses a $p$-cell $\tau_{\mathrm{p}}$ is the same $p$-dimensional "blob" that we adopted as a geometric object. The boundary $\partial \tau_{p}$ of a $p$-cell $\tau_{p}$ is the subset of $D$, which is linked by the preceding homeomorphism to the boundary $\partial B_{p}=\left\{\mathbf{x} \in \mathbb{R}^{p}:\|\mathbf{x}\|=1\right\}$ of $B_{p}$. A cell is internally (externally) oriented when we have selected as the positive orientation one of the two possible internal (external) orientations for it. According to our established convention, we will add a tilde to distinguish externally oriented cells $\tilde{\tau}_{p}$ from internally oriented cells $\tau_{p}$. To simplify the notation, in presenting new concepts we will usually refer to internally oriented cells. The results apply obviously to externally oriented objects as well.

In assembling the cells to form meshes, we must follow certain rules. These rules are dictated primarily by the necessity of relating in a certain way the physical quantities that are associated with the cells to those that are associated with their boundaries. Think, for example, of two adjacent 3-cells in a heat transfer problem; these cells can exchange heat through their common boundary, and we want to be able to associate this heat to a 2-cell belonging to the mesh. So that this goal can be achieved, the cells of the mesh must be properly joined (Fig. 21). In addition to this, since the heat balance equation for each 3-cell implies the heat associated with the boundary of the cell, this boundary must be paved with a finite number of 2-cells of the mesh. Finally,

${ }^{*}$ In actual numerical problems $p$-cells are usually nothing more than bounded, convex, oriented polyhedrons in $\mathbb{R}^{n}$. 
to avoid the association of a given global quantity to multiple cells, we should ensure that two distinct cells do not overlap.

A structure that complies with these requirements is an $n$-dimensional finite cell complex $K$. This is a finite set of cells with the following two properties:

1. The boundary of each $p$-cell of $K$ is the union of lower-dimensional cells of $K$ (these cells are called the proper $q$-dimensional faces of $\tau_{p}$, with $q$ ranging from from 0 to $p-1$; it is useful to consider a cell an improper face of itself).

2. The intersection of any two cells of $K$ is either empty or a (proper or improper) face of both cells.

This last requirement specifies the property of two cells' being "properly joined." We can, therefore, say that a finite cell complex $K$ is a finite collection of properly joined cells with the property that if $\tau_{p}$ is a cell of $K$, then every face of $\tau_{p}$ belongs to $K$. Note that the term face without specification of the dimension usually refers only to the $(p-1)$-dimensional faces. We say that a cell complex $K$ decomposes or is a subdivision of a domain $D$ (written $|K|=D$ ), if $D$ is equal to the union of the cells in $K$. The collection of the $p$-cells and of all cells of dimension lower than $p$ of a cell complex is called its $p$-skeleton. We will assume that our domains are always decomposable into finite cell complexes and assume that all our cell complexes are finite, even if this is not explicitly stated.

The requirement that the meshes be cell complexes may seem severe, for it implies proper joining of cells and covering of the entire domain without gaps or overlapping. A bit of reflection reveals, however, that this includes all structured and most nonstructured meshes, excluding only a minority of cases such as composite and nonconformal meshes. Nonetheless, this requirement will be relaxed later or, better, the concept of a cell will be generalized, so as to include structures that can be considered as derived from a cell complex by means of a limit process. This is the case in the finite element method and in some of its generalizations, for example, meshless methods. For now, however, we will base the next steps of our quest for a discrete representation of geometry and fields on the hypothesis that the meshes are cell complexes. Note that for time-dependent problems we assume that the cell complexes subdivide the whole space-time domain of the problem.

\section{Primary and Secondary Mesh}

The requirement of housing the global physical quantities of a problem implies that both objects with internal orientation and objects with external orientation must be available. Hence, two logically distinct meshes must be defined, one with internal orientation and the other with external orientation. Let us denote them with the symbols $K$ and $\tilde{K}$, respectively. Note that this requirement does 
not necessarily imply that two staggered meshes must always be used, for the two can share the same nonoriented geometric structure. There are, however, good reasons usually to also differentiate the two meshes geometrically. In particular, the adoption of two dual cell complexes as meshes endows the resulting discrete mathematical model with a number of useful properties. In an $n$-dimensional domain, the geometric duality means that to each $p$-cell $\tau_{p}^{i}$ of $K$ there corresponds a $(n-p)$-cell $\tilde{\tau}_{n-p}^{i}$ of $\tilde{K}$, and vice versa. Note that in this case we are purposely using the same index to denote the two cells, for this not only is natural but facilitates a number of proofs concerning the relation between quantities associated with the two dual complexes. We will denote with $n_{p}$ the number of $p$-cells of $K$ and with $\tilde{n}_{p}$ the number of $p$-cells of $\tilde{K}$. If the two $n$-dimensional cell complexes are duals, we have $n_{p}=\tilde{n}_{n-p}$. The names primal and dual meshes are often adopted for dual meshes. To allow for the case of nondual meshes, we will call primary mesh the internally oriented one and secondary mesh the externally oriented one. Note that the preceding discussion applies to the discretization of domains of any geometric dimension. Figure 22 shows an example of the two-dimensional case and dual grids, whereas Fig. 33 represents the same situation for the three-dimensional case.

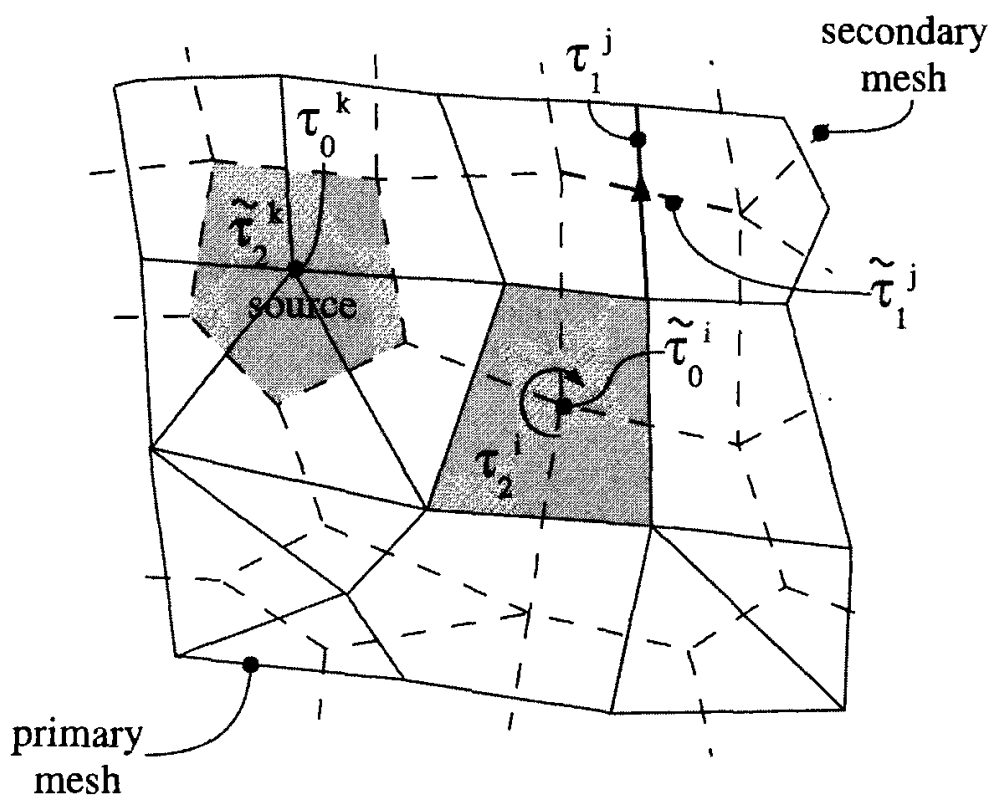

FIGURE 22. The primary and secondary meshes, for the case of a two-dimensional domain and dual meshes. Note that dual geometric objects share a common index and the symbol which assigns the orientation. All the geometric objects of both meshes must be considered as oriented. 


\section{Incidence Numbers}

Given a cell complex $K$, we want to give it an algebraic representation. Obviously, the mere list of cells of $K$ is not enough, for it lacks all information concerning the structure of the complex; that is, it does not tell us how the cells are assembled to form the complex. Since in a cell complex two cells can meet at most on common faces, we can represent the complex connectivity by means of a structure that collects the data about cell-face relations. We must also include information concerning the relative orientation of cells. This can be done as follows.

Each oriented geometric object induces an orientation on its boundary (Figs. 4 and 6); therefore, each $p$-cell of an oriented cell complex induces an orientation on its $(p-1)$-faces. We can compare this induced orientation with the default orientation of the faces as $(p-1)$-cells in $K$. Given the $i$ th $p$-cell $\tau_{p}^{i}$ and the $j$ th $(p-1)$-cell $\tau_{p-1}^{j}$ of a complex $K$, we define an incidence number $\left[\tau_{p}^{i}, \tau_{p-1}^{j}\right]$ as follows (Fig. 23):

$$
\left[\tau_{p}^{i}, \tau_{p-1}^{j}\right] \stackrel{\text { def }}{=} \begin{cases}0 & \text { if } \tau_{p-1}^{j} \text { is not a face of } \tau_{p}^{i} \\ +1 & \text { if } \tau_{p-1}^{j} \text { is a face of } \tau_{p}^{i} \text { and has the induced orientation } \\ -1 & \text { as above, but with opposite orientation }\end{cases}
$$

This definition associates with an $n$-dimensional cell complex $K$ a collection of $n$ incidence matrices

$$
\mathbf{D}_{p, p-1}=\left(\left[\tau_{p}^{i}, \tau_{p-1}^{j}\right]\right)
$$

where the index $i$ runs over all the $p$-cells of $K$, and $j$ runs over all the $(p-1)$ cells. We will denote by $\tilde{\mathbf{D}}_{p, p-1}$ the incidence matrices of $\tilde{K}$. In the particular case of dual cell complexes $K$ and $\tilde{K}$, if the same index is assigned to pairs of

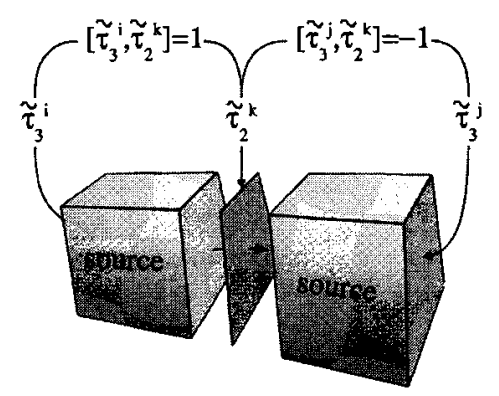

FIGURE 23. Incidence numbers describe the cell-face relations within a cell complex. All the other 3 -cells of the complex have 0 as their incidence number corresponding to the 2-cell $\tilde{\tau}_{2}^{k}$. 
dual cells, the following relations hold:

$$
\tilde{\mathbf{D}}_{p, p-1}=\mathbf{D}_{n-p+1, n-p}^{T}
$$

It can be proved with simple algebraic manipulations (Hocking and Young, 1988) that for an arbitrary $p$-cell $\tau_{p}$, the following relationship holds among incidence numbers:

$$
\sum_{i} \sum_{j}\left[\tau_{p}, \tau_{p-1}^{i}\right]\left[\tau_{p-1}^{i}, \tau_{p-2}^{j}\right]=0
$$

Even if at first sight this relation does not convey any geometric ideas, from it there follow many fundamental properties of the discrete operators that we shall introduce subsequently.

The set of oriented cells in $K$ and the set of incidence matrices constitute an algebraic representation of the structure of the cell complex. Browsing through the incidence matrices, we can know everything concerning the orientation and connectivity of cells within the complex. In particular, we can know if two adjacent cells induce on the common face opposite orientations, in which case they are said to have compatible or coherent orientation. This is an important concept, for it expresses algebraically the intuitive idea of two adjacent $p$-cells' having the same orientation (Figs. 23 and 24). Conversely, given an oriented $p$-cell, we can use this definition to propagate its orientation to neighboring $p$-cells [on orientable $n$-dimensional domains it is always possible to propagate the orientation of an $n$-cell to all the $n$-cells of the complex (Schutz, 1980)].
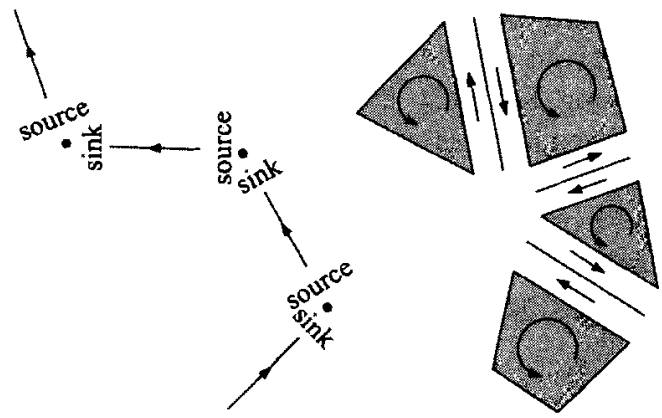

FIGURE 24. Two adjacent cells have compatible orientation if they induce on the common face opposite orientations. The concept of induced orientation can be used to propagate the orientation of a $p$-cell to neighboring $p$-cells. 


\section{Chains}

Now that we know how to represent algebraically the cell complex, which discretizes the domain, we want to construct a machinery to represent generic parts of it. This means that we want to represent an assembly of cells, each with a given orientation and weight of our choice. A first requirement for this task is the ability to represent cells with the default orientation and cells with the opposite one. This is most naturally achieved by denoting a cell with its default orientation with $\tau_{p}$ and one with the opposite orientation with $-\tau_{p}$. We can then represent a generic $p$-dimensional domain $\mathbf{c}_{p}$ composed by $p$-cells of the complex $K$ as a formal sum,

$$
\mathbf{c}_{p}=\sum_{i=1}^{n_{p}} w_{i} \tau_{p}^{i} \quad \tau_{p}^{i} \in K
$$

where the coefficient $w_{i}$ can take the value $0,+1$, or -1 , to denote a cell of the complex not included in $\mathbf{c}_{p}$, or included in it with the default orientation or its opposite, respectively. This formalism, therefore, allows the algebraic representation of discrete subdomains as "sums" of cells.

We now make a generalization, allowing the coefficients of the formal sum [Eq. (91)] to take arbitrary real values $w_{i} \in \mathbb{R}$. To preserve the representation of the orientation inversion as a sign inversion, we assume that the following property holds true:

$$
w_{i}\left(-\tau_{p}^{i}\right)=-w_{i} \tau_{p}^{i}
$$

With this extension, we can represent oriented $p$-dimensional domains in which each cell is weighted differently. This entity is analogous, in a discrete setting, to a subdomain with a weight function defined on it; thus it will be useful in order to give a geometric interpretation to the discretization strategies of numerical methods, such as finite elements, which make use of weight functions. In algebraic topology, given a cell complex $K$, a formal sum like Eq. (91), with real weights satisfying Eq. (92), is called a p-dimensional chain with real coefficients, or simply a $p$-chain $\mathbf{c}_{p}$ (Fig. 25).

If it is necessary to specify explicitly the coefficient space for the weights $w_{i}$ and the cell complex on which a particular chain is built, we write $\mathbf{c}_{p}(K, \mathbb{R})$. We can define in an obvious way an operation of addition of chains defined on the same complex, and one of multiplication of a chain by a real number $\lambda$, as follows:

$$
\begin{gathered}
\mathbf{c}_{p}+\mathbf{c}_{p}^{\prime}=\sum_{i} w_{i}^{\prime} \tau_{p}^{i}+\sum w_{i} \tau_{p}^{i}=\sum_{i}\left(w_{i}+w_{i}^{\prime}\right) \tau_{p}^{i} \\
\lambda \mathbf{c}_{p}=\lambda \sum_{i} w_{i} \tau_{p}^{i}=\sum_{i}\left(\lambda w_{i}\right) \tau_{p}^{i}
\end{gathered}
$$




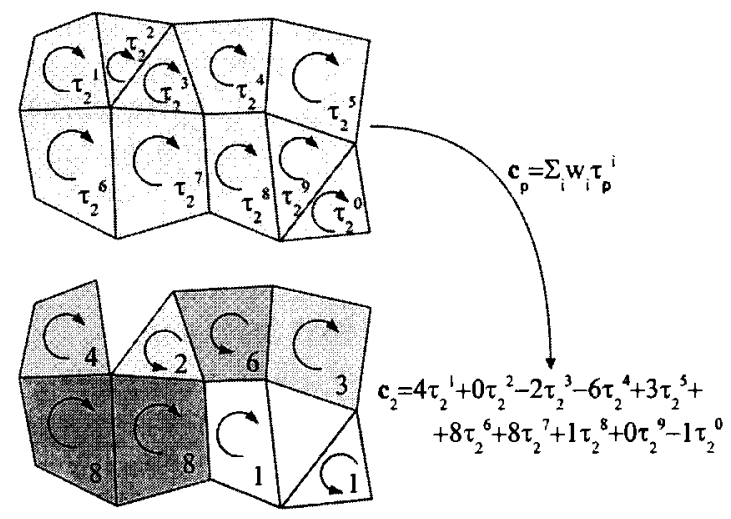

FIGURE 25. Given an oriented cell complex (top), a $p$-chain (bottom) represents a weighted sum of oriented $p$-cells. The weights are represented as shades of gray. Notice that negative weights make the corresponding cell appear in the chain with its orientation reversed with respect to the default orientation of the cell in the cell complex.

With these definitions the set of $p$-chains with real coefficients on a complex $K$ becomes a vector space $C_{p}(K, \mathbb{R})$ over $\mathbb{R}$, often written simply as $C_{p}(K)$ or $C_{p}$. The dimension of this space is the number $n_{p}$ of $p$-cells in $K$. Note that each $p$-cell $\tau_{p}$ can be considered an elementary $p$-chain $1 \cdot \tau_{p}$. These elementary $p$-chains constitute a natural basis in $C_{p}$, which permits the representation of a chain by the $n_{p}$-tuple of its weights:

$$
\mathbf{c}_{p}=\left(w_{1}, w_{2}, \ldots, w_{n_{p}}\right)
$$

Working with the natural basis, we can easily define linear operators on chains as linear extensions of their action on cells. In particular, this is the case for the definition of the boundary of a chain.

\section{The Boundary of a Chain}

The boundary $\partial \tau_{p}$ of a cell $\tau_{p}$ is by definition the collection of its faces, endowed with the induced orientation (Figs. 4 and 6). Remembering the definition of the incidence numbers, we can write

$$
\partial \tau_{p}=\sum_{j=1}^{n_{p-1}}\left[\tau_{p}, \tau_{p-1}^{j}\right] \tau_{p-1}^{j}
$$

where the index $j$ runs on all the $(p-1)$-cells of the complex. Note that Eq. (96) gives to a geometric operation an algebraic representation based uniquely on incidence matrices. Since the $p$-cells constitute a natural basis 
for the space of $p$-chains, we can extend linearly the definition of $\partial$ to an operator-the boundary operator-acting on arbitrary $p$-chains, as follows:

$$
\partial \mathbf{c}_{p}=\partial\left(\sum_{i} w_{i} \tau_{p}^{i}\right)=\sum_{i} w_{i} \partial \tau_{p}^{i}
$$

Thus the boundary of a $p$-chain is a $(p-1)$-chain, and $\partial$ is a linear mapping $\partial: C_{p}(K) \rightarrow C_{p-1}(K)$ of the space of $p$-chains into that of $(p-1)$-chains. It can be proved (Hocking and Young, 1988), by using Eq. (90), that for any chain $\mathbf{c}_{p}$ the following identity holds true:

$$
\partial\left(\partial \mathbf{c}_{p}\right)=0
$$

That is, the boundary of a chain has no boundary, a result that, when applied to elementary chains (i.e., to $p$-cells), satisfies our geometric intuition.

The boundary of a cell defined by Eq. (96) coincides practically with the usual geometric idea of the boundary of a domain, complemented by the fact that the faces are endowed with the induced orientation. The calculation of the boundary of a chain defined by Eq. (97) can instead give a nonobvious result. Let us consider $p$-chains built with a set of cells that form a $p$-dimensional domain (Fig. 26). For some chains of this kind, it may happen that the result of the application of the boundary operator includes $(p-1)$-cells that we typically do not consider as belonging to the boundary of the domain. In fact, it turns out

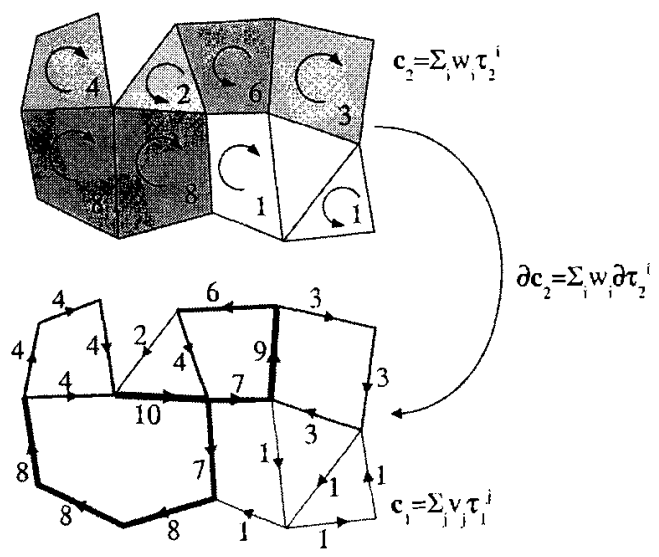

Figure 26. Given a $p$-chain $\mathbf{c}_{p}$ (top), its boundary $\partial \mathbf{c}_{p}$ is a $(p-1)$-chain (bottom) that usually includes internal "vestiges" with respect to what we are used to considering the boundary of the domain spanned by the $p$-cells appearing in the $p$-chain. The weights of 2-cells are represented as shades of gray and those of 1-cells by the thickness of lines. 
that this represents the rule, not the exception, since each "internal" $(p-1)$-cell of the domain $\mathbf{c}_{p}$ appears in Eq. (97), unless the sum of the weights received by it from the $p$-cells of which it is a face [the so-called cofaces of the $(p-1)$-cell] vanishes. Obviously, this vanishing is true only for particular sets of weights; that is, for particular chains. Later, we shall build a correspondence between chains and weighted domains. In that context, the boundary of a weighted domain will be defined, and the result will turn out to be confined to the traditional boundary only for particular weight functions.

\section{B. Fields}

A consequence of our traditional mathematical education is that when we hear the word field we tend to think immediately of its representation in terms of some kind of field function; that is, of some continuous representation. If we refrain from this premature association, we can easily recognize that the transition from what is observed to this kind of representation requires a nontrivial abstraction. In practice, we can measure only global quantities; that is, quantities related to macroscopic $p$-dimensional space-time subdomains of a given domain. It is, however, natural to imagine that we could potentially perform an infinite number of measurements for all the possible subdomains. We then conceive this collection of possible measurements as a unique entity, which we call the field, and we represent this entity mathematically in a way that permits the modeling of these measurements, for example, as a field function that can be integrated on arbitrary $p$-dimensional subdomains.

Consider now a domain in which we have built a mesh, say, a cell complex $K$. By so doing, we have selected a particular collection of subdomains, the cells of the complex $K$. Consequently we must (and can) deal only with the global quantities associated with these subdomains. The fields will manifest themselves on this mesh as collections of global quantities associated with these cells only. Of course, this association will be sensitive to the orientation and linear on cell assembly. This, in essence, is the idea behind the representation of field on discretized domains in terms of cochains.

\section{Cochains}

Given an oriented cell complex $K$ and an (algebraic) field $\mathcal{F}$, consider a function $\mathbf{c}^{p}$ which assigns to each cell $\tau_{p}^{i}$ of $K$ (thought of as an elementary chain) an element $c^{i}$ of $\mathcal{F}$, written

$$
\left(\tau_{p}^{i}, \mathbf{c}^{p}\right)=c^{i}
$$


and is linear on the operation of cell assembly represented by chains; that is, it satisfies

$$
\left(\mathbf{c}_{p}, \mathbf{c}^{p}\right)=\left(\sum w_{i} \tau_{p}^{i}, \mathbf{c}^{p}\right)=\sum w_{i}\left(\tau_{p}^{i}, \mathbf{c}^{p}\right)
$$

This function $\mathbf{c}^{p}$ is called a $p$-dimensional cochain, or simply $p$-cochain $\mathbf{c}^{p}$. It can be written as $\mathbf{c}^{p}(K, \mathcal{F})$ or $\mathbf{c}^{p}(K)$ to designate explicitly the cell complex and the algebraic field involved in the definition [when the complex is externally oriented, we will write $\mathbf{c}^{p}(\tilde{K})$ if the complex is explicitly mentioned, and $\tilde{\mathbf{c}}^{p}$ if it is not]. We will call ordinary cochains those defined on an internally oriented cell complex, and twisted those defined on an externally oriented one (Burke, 1985; Teixeira and Chew, 1999b).

We can readily see that this definition contains the essence of what we said previously concerning the action of physical fields on domains partitioned into cell complexes. The cochain, like a field, associates a value with each cell, and the association is additive on cell assembly. Note that from Eq. (100) it follows that

$$
\left(-\tau_{p}, \mathbf{c}^{p}\right)=-\left(\tau_{p}, \mathbf{c}^{p}\right)
$$

That is, as expected, the value assumed by a cochain on a cell changes sign with the inversion of the orientation of the cell. Thus, the only thing that must be added to the mathematical definition of a cochain to make it suitable for the representation of fields is the attribution of a physical dimension to the values associated with cells. With this further attribution the values can be interpreted as global physical quantities (which-we stress again-need not be scalars) and the corresponding entity can be called a physical p-cochain. All cochains considered in this work must be considered physical cochains, even if the qualifier "physical" is omitted.

From Eq. (100) we see that a cochain $\mathbf{c}^{p}$ is actually a linear mapping $\mathbf{c}^{p}$ : $C_{p}(K) \rightarrow \mathcal{F}$ of the space of chains $C_{p}(K)$ into the algebraic field $\mathcal{F}$, which assigns to each chain $\mathbf{c}_{p}$ a value

$$
\left(\mathbf{c}_{p}, \mathbf{c}^{p}\right)
$$

This representation emphasizes the equal role of the chain and of the cochain in the pairing. To assist our intuition, we can think of Eq. (102) as a discrete counterpart of the integral of a field function on a weighted domain, and this can suggest the following alternative representation for the pairing (Bamberg and Sternberg, 1988):

$$
\left(\mathbf{c}_{p}, \mathbf{c}^{p}\right) \equiv \int_{\mathbf{c}_{p}} \mathbf{c}^{p}
$$


We can define the sum of two cochains and the product of a cochain by an element of $\mathcal{F}$, as follows:

$$
\begin{aligned}
\left(\mathbf{c}_{p}, \mathbf{c}^{p}+\mathbf{c}^{p^{\prime}}\right) & =\left(\mathbf{c}_{p}, \mathbf{c}^{p}\right)+\left(\mathbf{c}_{p}, \mathbf{c}^{p^{\prime}}\right) \\
\left(\lambda \mathbf{c}_{p}, \mathbf{c}^{p}\right) & =\lambda\left(\mathbf{c}_{p}, \mathbf{c}^{p}\right)
\end{aligned}
$$

This definition transforms the set of cochains in a vector space $C^{p}(K, \mathcal{F})$ over $\mathcal{F}$, usually written simply as $C^{p}(K)$ or $C^{p}$. A natural basis for this vector space is constituted by the elementary $p$-cochains which assign the unity of $\mathcal{F}$ to a $p$-cell and the null element of $\mathcal{F}$ to all other $p$-cells of the complex. The dimension of $C^{p}(K)$ is, therefore, the number $n_{p}$ of $p$-cells in $K$, and on the natural basis we can represent uniquely a cochain as the $n_{p}$-tuple of its values on cells:

$$
\mathbf{c}^{p}=\left(c^{1}, c^{2}, \ldots, c^{n_{p}}\right)^{T} \quad c^{i}=\left(\tau_{p}^{i}, \mathbf{c}^{p}\right) \in \mathcal{F}
$$

With this representation, and with the corresponding one for a chain [Eq. (95)], the pairing of a chain and a cochain is given by

$$
\left(\mathbf{c}_{p}, \mathbf{c}^{p}\right)=\sum_{i=1}^{n_{p}} w_{i} c^{i}
$$

In the case of a physical cochain, the natural representation would be an $n_{p}$-tuple of global physical quantities associated with $p$-cells. For example, in a heat transfer problem the heat content 3-cochain $\tilde{\mathbf{Q}}_{c}^{3}$ is represented by the $\tilde{n}_{3}$-tuple of the heat contents of the 3-cells $\tilde{\tau}_{3}$ of the cell complex $K$, which discretizes the domain:

$$
\tilde{\mathbf{Q}}_{c}^{3}=\left(Q_{c}^{1}, Q_{c}^{2}, \ldots, Q_{c}^{\tilde{n}_{3}}\right)^{T}
$$

where

$$
Q_{c}^{i}=Q_{c}\left(\tilde{\tau}_{3}^{i}\right)=\left(\tilde{\tau}_{3}^{i}, \tilde{\mathbf{Q}}_{c}^{3}\right)
$$

The heat $Q_{c}$ associated with a chain $\tilde{\mathbf{c}}_{3}=\sum_{i=1}^{\tilde{n}_{3}} w_{i} \tilde{\tau}_{3}^{i}$ corresponds, therefore, to

$$
Q_{c}=\left(\tilde{\mathbf{c}}_{3}, \tilde{\mathbf{Q}}_{c}^{3}\right)=\sum_{i=1}^{\tilde{n}_{3}} w_{i} Q_{c}^{i}
$$

Note the similarity with a weighted integral:

$$
Q_{c}=\int_{\tilde{V}} w q_{c}
$$

Using the concept of cochain, we can redraw the classification diagrams of physical quantities for a discretized domain, substituting the field functions 


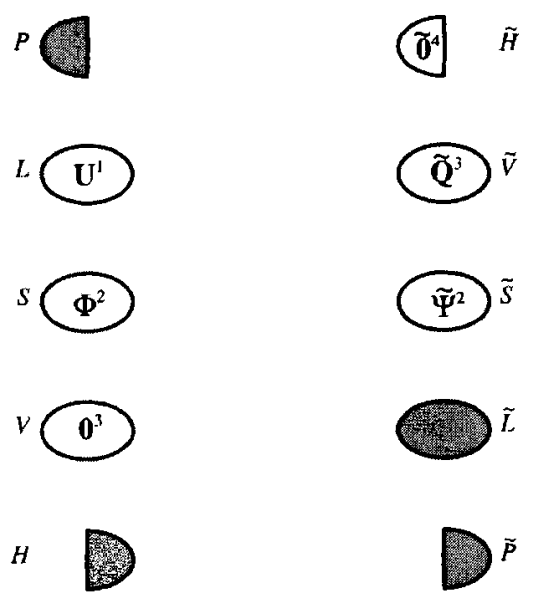

FIGURE 27. The Tonti classification diagram of global electromagnetic physical quantities in terms of cochains. Note the presence of two null cochains, corresponding to the absence of magnetic flux production and to the absence of electric charge production.

with the corresponding cochains. For example, in electromagnetism we have the 1-cochain $\mathbf{U}^{1}$ of electromagnetic potential; the 2-cochains $\Phi^{2}$ and $\tilde{\Psi}^{2}$ of magnetic flux and electric flux, respectively; and the 3-cochain $\tilde{\mathbf{Q}}^{3}$ of electric charge (to which we must add the null 3 -cochain $0^{3}$ of magnetic flux production and the null 4-cochain $\tilde{\mathbf{0}}^{4}$ of electric charge production). The corresponding classification diagram is depicted in Figure 27.

Remark III.I It is sometimes argued that on finite complexes, cochains and chains coincide, since both associate numbers with a finite number of cells (Hocking and Young, 1988). Even disregarding that the numbers associated by chains are dimensionless multiplicities whereas those associated by cochains are physical quantities, the two concepts are quite different. Chains can be seen as functions which associate numbers with cells. The only requirement is that the number changes sign if the orientation of the cell is inverted. Note that no mention is made of values associated with collections of cells, nor could it be made, for this concept is still undefined. Before the introduction of the concept of chain we have at our disposal only the bare structure of the complex-the set of cells in the complex and their connectivity as described by the incidence matrices. It is the very definition of chain which provides the concept of an assembly of cells. Only at this point can the cochains be defined, which associate numbers not only with single cells, as chains do, but also with assemblies of cells. This association is required to be not only orientation dependent, but also linear with respect to the assembly of cells represented by 
chains. This extension from weights associated with single cells to quantities associated with assemblies of cells is not trivial and makes cochains a very different entity from chains, even on finite cell complexes.

\section{Limit Systems}

The idea of the field as a collection of its manifestations in terms of cochains on the cell complexes that subdivide the domain of a problem, finds a representation in certain mathematical structures called limit systems. The basic idea is that we can consider in a domain $D$ the set $\mathcal{K}$ of all the cell complexes that can be built on it (with the kind of orientation that suits the field at hand). We can then form a collection of all the corresponding physical $p$-cochains on the complexes in $\mathcal{K}$. This collection can be considered intuitively the collection of all the possible measurements for all possible field configurations on $D$.

Next we want to partition this collection of cochains into sets, with each set including only measurements that derive from a given field configuration. We define for this task a selection criterion based on the additivity of global quantities. This criterion is the relation that links the cochains within each set and allows our considering each of these sets a new entity, which in our interpretation is a particular field configuration thought of as a collection of its manifestations in terms of cochains. We can define operations between fields, and operators acting on them, deriving naturally from the corresponding ones defined for cochains. For example, we can define addition of fields and the analogous of traditional differential operators (gradient, curl, and divergence) in intuitive discrete terms. This allows an easy transition from the discrete, observable properties to the corresponding continuous abstractions.

The reader is warned that the rest of this section is abstract, as compared with the prevailing style of the present work. The details, however, can be skipped at first reading, since only the main ideas are required in the sequel. The point is not to give a sterile formalization to the ideas presented so far, but to provide conceptual tools for the representation of the link existing between discrete and continuous models.

Let us now address the mathematics. Consider the set $\mathcal{K}=\left\{K_{\alpha}\right\}$ of all cell complexes which subdivide a domain $D$. In this case, the complexes are internally oriented, but they could be externally oriented ones as well. We will say that a complex $K_{\beta}$ is a refinement of $K_{\alpha}$-written $K_{\alpha}<K_{\beta}$-if each cell of $K_{\alpha}$ is a union of cells of $K_{\beta}$. The set $\mathcal{K}$ is partially ordered by the relation <, and for any pair of complexes $K_{\alpha}, K_{\beta}$ there exists a complex $K_{\gamma}$ in $\mathcal{K}$, which is a refinement of both (remember that our domains are homeomorphic to polytopes) (Whitney, 1957). This property makes of $\mathcal{K}$ a directed set (Eilenberg and Steenrod, 1952; Hocking and Young, 1988). 
For each complex $K_{\alpha}$ in $\mathcal{K}$ let us consider the set $C^{p}\left(K_{\alpha}\right)$ of all the physical $p$-cochains on $K_{\alpha}$, with a given physical dimension [with our choice of the space where the cochains take their values, $C^{p}\left(K_{\alpha}\right)$ is a vector space, but for simplicity, we will consider only the group operation in the sequel]. Whenever $K_{\alpha}<K_{\beta}$ in $\mathcal{K}$, there is a transformation $f_{K_{\alpha} K_{\beta}}: C^{p}\left(K_{\beta}\right) \rightarrow C^{p}\left(K_{\alpha}\right)$ of $C^{p}\left(K_{\beta}\right)$ into $C^{p}\left(K_{\alpha}\right)$ which, to each cochain $\mathbf{c}^{p}\left(K_{\beta}\right)$, associates the cochain $\mathbf{c}^{p}\left(K_{\alpha}\right)$ taking on each cell $\tau_{p}$ of $K_{\alpha}$ the sum (with proper signs, to take care of orientation) of the values taken by $\mathbf{c}^{p}\left(K_{\beta}\right)$ on the cells of $K_{\beta}$ which compose $\tau_{p}$. Physically speaking the transformations $f_{K_{\alpha} K_{\beta}}$ are based on the additivity of the global physical quantities upon cell assembly. These transformations satisfy the following properties:

- $f_{K_{\alpha} K_{\alpha}}$ is the identity transformation for each $K_{\alpha}$ in $\mathcal{K}$.

- $f_{K_{\alpha} K_{\beta}} f_{K_{\beta} K_{\gamma}}=f_{K_{\alpha} K_{\gamma}}$ whenever $K_{\alpha}<K_{\beta}<K_{\gamma}$.

If $F$ denotes the collection $\left\{f_{K_{\alpha} K_{\beta}}\right\}$ of all such transformations, and $\mathcal{C}^{p}$ the collection $\left\{C^{p}\left(K_{\alpha}\right), K_{\alpha} \in \mathcal{K}\right\}$ of all the physical $p$-cochains homogeneous relative to the physical nature of the field, on cell complexes in $\mathcal{K}$, the pair $\left\{\mathcal{C}^{p}, F\right\}$ is called an inverse limit system over the directed set $\mathcal{K}$. Each set $C^{p}\left(K_{\alpha}\right)$ in the collection $\mathcal{C}^{p}$ is a group, and each $f_{K_{\alpha} K_{\beta}}$ in $F$ is a homomorphism.

The inverse limit group $C^{p}\left(K_{\infty}\right)$ of the system $\left\{\mathcal{C}^{p}, F\right\}$ is the subgroup of the direct sum $\sum_{\mathcal{K}} C^{p}\left(K_{\alpha}\right)$ consisting of all sets $\left\{\mathbf{c}^{p}\left(K_{\alpha}\right)\right\}$-one element from each group $C^{p}\left(K_{\alpha}\right)$-for which $f_{K_{\alpha} K_{\beta}}\left(\mathbf{c}^{p}\left(K_{\beta}\right)\right)=\mathbf{c}^{p}\left(K_{\alpha}\right)$ whenever $K_{\alpha}<K_{\beta}$ in $\mathcal{K}$. The group operation in $C^{p}\left(K_{\infty}\right)$ is defined naturally by the formula

$$
\left\{\mathbf{c}^{p}\left(K_{\alpha}\right)\right\}+\left\{\mathbf{c}^{p}\left(K_{\alpha}\right)\right\}=\left\{\mathbf{c}^{p}\left(K_{\alpha}\right)+\mathbf{c}^{p}\left(K_{\alpha}\right)\right\}
$$

where the sum on the right indicates the group operation in each $C^{p}\left(K_{\alpha}\right)$. For each $K_{\beta}$ in $\mathcal{K}$ there is a natural projection $\pi_{K_{\beta}}: C^{p}\left(K_{\infty}\right) \rightarrow C^{p}\left(K_{\beta}\right)$, defined by $\pi_{K_{\beta}}\left(\left\{\mathbf{c}^{p}\left(K_{\alpha}\right)\right\}\right)=\mathbf{c}^{p}\left(K_{\beta}\right)$. Each projection $\pi_{K_{\alpha}}$ is a homomorphism.

As anticipated we can interpret all this physically as follows. Each element $\left\{\mathbf{c}^{p}\left(K_{\alpha}\right)\right\}$ of the inverse limit group $C^{p}\left(K_{\infty}\right)$ represents a physical field defined in the domain $D$, which associates physical quantities to $p$-dimensional geometric objects (let us call it a physical p-field, or simply $p$-field). The set $\left\{\mathbf{c}^{p}\left(K_{\alpha}\right)\right\}$ is the collection of its manifestations [in terms of cochains $\mathbf{c}^{p}\left(K_{\alpha}\right)$ ] on the cell complexes $K_{\alpha} \in \mathcal{K}$, which decompose $D$. The cochains that correspond to a given field can be recognized for they are linked by the functions in $F$. The group operation in $C^{p}\left(K_{\infty}\right)$ is the addition of fields. The projection $\pi_{K_{\beta}}$ associates with each cell complex $K_{\beta}$ and with each field $\left\{\mathbf{c}^{p}\left(K_{\alpha}\right)\right\}$ the corresponding cochain $\mathbf{c}^{p}\left(K_{\beta}\right)$. In the previous example we would have on a complex $K_{\alpha}$ the heat content cochains $\tilde{\mathbf{Q}}_{c}\left(K_{\alpha}\right)$, and each set $\left\{\tilde{\mathbf{Q}}_{c}\left(K_{\alpha}\right)\right\}$ of the inverse limit group $Q_{c}\left(K_{\infty}\right)$ would be a particular heat content field. 
To complete this exposition of limit systems, we will now anticipate some ideas, related to the action of an operator between cochain spaces, that will be introduced in the next section. For each cell complex $K_{\alpha}$ we will define an operator $\delta_{K_{\alpha}}$-the coboundary operator-which will be used to write topological laws in discrete form. This is a homomorphism of $C^{p}\left(K_{\alpha}\right)$ into $C^{p+1}\left(K_{\alpha}\right)$. Given the two inverse limit systems $\left\{\mathcal{C}^{p}, F\right\}$ and $\left\{\mathcal{C}^{p+1}, F^{\prime}\right\}$ over the directed set $\mathcal{K}$ [i.e., the inverse limit system constructed with the $p$-cochains and the $(p+1)$-cochains on the cell complexes which decompose $D$ ], we define a transformation $\delta$ of $\left\{\mathcal{C}^{p}, F\right\}$ into $\left\{\mathcal{C}^{p+1}, F^{\prime}\right\}$ based on the action of $\delta_{K_{\alpha}}$. The transformation $\delta$ consists, for each $K_{\alpha}$ in $\mathcal{K}$, of the transformation $\delta_{K_{\alpha}}$ with the condition that whenever $K_{\alpha}<K_{\beta}$ in $\mathcal{K}$, the commutative relation $\delta_{K_{\alpha}} f_{K_{\alpha} K_{\beta}}=f_{K_{\alpha} K_{\beta}}^{\prime} \delta_{K_{\beta}}$ holds true, such that the sum on $p$-cells goes into the sum on $(p+1)$-cells. Such a transformation $\delta$ of $\left\{\mathcal{C}^{p}, F\right\}$ into $\left\{\mathcal{C}^{p+1}, F^{\prime}\right\}$ induces a homomorphism $\delta_{\infty}$ of the inverse limit groups $C^{p}\left(K_{\infty}\right)$ into $C^{p+1}\left(K_{\infty}\right)$ as follows. If $\left\{\mathbf{c}^{p}\left(K_{\alpha}\right)\right\}$ is an element of $\left\{\mathcal{C}^{p}, F\right\}$, and $K_{\alpha}$ in $\mathcal{K}$ is given, set $\mathbf{c}^{p+1}\left(K_{\alpha}\right)=\delta_{K_{\alpha}}\left(\mathbf{c}^{p}\left(K_{\alpha}\right)\right)$. Note that if $K_{\alpha}<K_{\beta}$ the preceding commutative relation tells us that $f_{K_{\alpha} K_{\beta}}^{\prime}\left(\mathbf{c}^{p+1}\left(K_{\beta}\right)\right)=\mathbf{c}^{p+1}\left(K_{\alpha}\right)$. Thus $\left\{\mathbf{c}^{p+1}\left(K_{\alpha}\right)\right\}$ is an element of $C^{p+1}\left(K_{\infty}\right)$. We define $\delta_{\infty}\left(\left\{\mathbf{c}^{p}\left(K_{\alpha}\right)\right\}\right)=\left\{\mathbf{c}^{p+1}\left(K_{\alpha}\right)\right\}$. Since each element $\left\{C^{p}\left(K_{\alpha}\right)\right\}$ of the inverse limit group $C^{p}\left(K_{\infty}\right)$ represents a $p$-field defined in the domain $D, \delta_{\infty}$ represents an operator which transforms a $p$-field in a $(p+1)$ field on $D$. We will see that it can be considered a way to define "differential" operators without the use of derivatives.

All this shows how the idea of field can be considered a limit concept abstracted from a collection of discrete manifestations of the field on cell complexes or, if you prefer, from a collection of possible measurements of global physical quantities. Correspondingly, a physical law concerning a field can be considered a collection of relations between the cochains that constitute the field. Note that the idea of field is an abstraction that remains at a higher logical level than that of actual measurements. So, we must take care not to treat a single cochain on a particular cell complex as if it were a field (which is instead a class of cochains), for this would be an error of logical typing (such as eating the menu card instead of the dinner) (Bateson, 1972).

\section{Topological Laws}

Equipped with the concept of the cochain, we are now in a position to give topological laws a discrete representation. Previously, we derived the topological laws of electromagnetism in discrete form [Eqs. (56) through (62)]. The fundamental property of all these relations - shared by all topological laws-is that they equate a global physical quantity associated with a geometric object to 
another global quantity associated with its boundary. This appears even more clearly in the space-time formulation of the same laws [Eqs. (63) through (66), repeated next for easy reference]:

$$
\begin{aligned}
\phi(\partial \mathcal{V}) & =0(\mathcal{V}) \\
Q(\partial \tilde{\mathcal{H}}) & =0(\tilde{\mathcal{H}}) \\
\mathcal{U}(\partial \mathcal{S}) & =\phi(\mathcal{S}) \\
\psi(\partial \tilde{\mathcal{V}}) & =Q(\tilde{\mathcal{V}})
\end{aligned}
$$

If the domain is meshed with the primary and secondary cell complexes we will have to substitute the generic geometric objects appearing in Eqs. (113) through (116) with the cells of $K$ and $\tilde{K}$. Equations (113) through (116), then, become

$$
\begin{array}{ll}
\phi\left(\partial \tau_{3}\right)=0\left(\tau_{3}\right) & \forall \tau_{3} \in K \\
Q\left(\partial \tilde{\tau}_{4}\right)=0\left(\tilde{\tau}_{4}\right) & \forall \tilde{\tau}_{4} \in \tilde{K} \\
\mathcal{U}\left(\partial \tau_{2}\right)=\phi\left(\tau_{2}\right) & \forall \tau_{2} \in K \\
\psi\left(\partial \tilde{\tau}_{3}\right)=Q\left(\tilde{\tau}_{3}\right) & \forall \tilde{\tau}_{3} \in \tilde{K}
\end{array}
$$

Equations (117) through (120) have simple interpretations. For example, Eq. (120) says that the charge associated with each 3-cell of the secondary mesh $\tilde{K}$ equals the electric flux associated with the boundary of the 3-cell.

\section{The Coboundary Operator}

Each of Eqs. (117) through (120) is a list of equivalences between global quantities. We can ask if the discrete representation of fields in terms of cochains can be used to write this list in a more compact way. A first step in this direction consists of writing each global quantity as a chain-cochain pairing. For example, Eq. (120) becomes

$$
\left(\partial \tilde{\tau}_{3}, \tilde{\Psi}^{2}\right)=\left(\tilde{\tau}_{3}, \tilde{\mathbf{Q}}^{3}\right) \quad \forall \tilde{\tau}_{3} \in \tilde{K}
$$

However, this is still a list of equivalences of global quantities, not an equivalence of two cochains. In addition, we cannot equate directly the two cochains $\tilde{\Psi}^{2}$ and $\tilde{\mathbf{Q}}^{3}$ because a domain and its boundary have different geometric dimensions, and the corresponding cochains belong consequently to two different spaces, in this case to $C^{2}(\tilde{K})$ and $C^{3}(\tilde{K})$, respectively. We can circumvent this problem by defining an operator $\delta$, which, on a given cell complex $K$, transforms a $p$-cochain $\mathbf{c}^{p}$ into a $(p+1)$-cochain $\delta \mathbf{c}^{p}$, which satisfies the following 
relation:

$$
\left(\tau_{p+1}, \delta \mathbf{c}^{p}\right) \stackrel{\text { def }}{=}\left(\partial \tau_{p+1}, \mathbf{c}^{p}\right) \quad \forall \tau_{p+1} \in K
$$

We can extend this definition linearly to arbitrary chains, as follows:

$$
\left(\mathbf{c}_{p+1}, \delta \mathbf{c}^{p}\right) \stackrel{\text { def }}{=}\left(\partial \mathbf{c}_{p+1}, \mathbf{c}^{p}\right)
$$

In this way we have defined an operator $\delta$, which is a linear mapping of the cochain space $C^{p}(K)$ into $C^{p+1}(K)$ (to see how this operator acts on combinations of cochains, simply substitute $\mathbf{c}^{p}+\mathbf{c}^{p^{\prime}}$ or $\lambda \mathbf{c}^{p}$ for $\mathbf{c}^{p}$ on the left side and observe the effects on the right side). This operator is called the coboundary operator and is just the operator needed to construct the linear transformation $\delta_{\infty}$ of the inverse limit system $C^{p}\left(K_{\infty}\right)$ into $C^{p+1}\left(K_{\infty}\right)$ [i.e., the transformation of $p$-fields into $(p+1)$-fields] anticipated in the final paragraphs of the section devoted to limit systems (Section III.B.2).

Let us apply the definition of this new operator to Eq. (121). Particularizing Eq. (122) for the electric flux cochain $\tilde{\Psi}^{2}$, we have

$$
\left(\tilde{\tau}_{3}, \delta \tilde{\Psi}^{2}\right) \stackrel{\text { def }}{=}\left(\partial \tilde{\tau}_{3}, \tilde{\Psi}^{2}\right) \quad \forall \tau_{3} \in K
$$

which, substituted in Eq. (121), gives

$$
\left(\tilde{\tau}_{3}, \delta \tilde{\Psi}^{2}\right)=\left(\tilde{\tau}_{3}, \tilde{\mathbf{Q}}^{3}\right) \quad \forall \tilde{\tau}_{3} \in \tilde{K}
$$

Since Eq. (125) asserts the identity of the components of the two cochains in the natural basis representation, it affirms, in fact, the identity of the two cochains. We can, therefore, thanks to the definition of the operator $\delta$, write the topological law [Eq. (116)] in terms of cochains only, as follows:

$$
\delta \tilde{\Psi}^{2}=\tilde{\mathbf{Q}}^{3}
$$

The definition [Eq. (123)] of the coboundary operator may seem abstract. However, it has a very intuitive meaning that can be exemplified as follows (Tonti, 1975). Equation (124) can be rewritten by substituting $\partial \tilde{\tau}_{3}$ with its expression in terms of incidence numbers. Exploiting the linearity of the chaincochain pairing, after some reordering of terms, gives

$$
\left(\tilde{\tau}_{3}, \delta \tilde{\Psi}^{2}\right)=\sum_{i}\left[\tilde{\tau}_{3}, \tilde{\tau}_{2}^{i}\right]\left(\tilde{\tau}_{2}^{i}, \tilde{\Psi}^{2}\right)
$$

More generally, Eq. (122) becomes

$$
\left(\tau_{p+1}, \delta \mathbf{c}^{p}\right)=\sum_{i}\left[\tau_{p+1}, \tau_{p}^{i}\right]\left(\tau_{p}^{i}, \mathbf{c}^{p}\right)
$$

This means that the coboundary operator operates on a $p$-cochain $\mathbf{c}^{p}$ and builds a $(p+1)$-cochain $\delta \mathbf{c}^{p}$, which assumes on each $(p+1)$-cell $\tau_{p+1}$ the global 


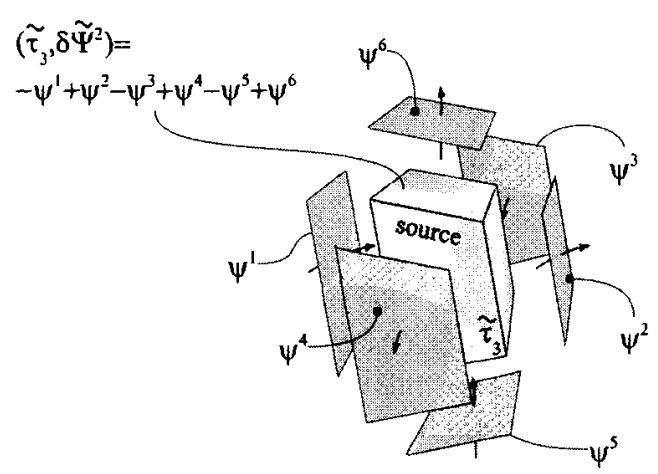

Figure 28. The coboundary of a $p$-cochain is a $(p+1)$-cochain, which assigns to each $(p+1)$-cell the sum of the values that the $p$-cochain assigns to the $p$-cells which form the boundary of the $(p+1)$-cell. Note that each quantity appears in the sum multiplied by the corresponding incidence number.

physical quantity associated by $\mathbf{c}^{p}$ with the boundary of $\tau_{p+1}$. This value is equal to the sum of the physical quantities associated by $\mathbf{c}^{p}$ with the faces of $\tau_{p+1}$ endowed with the induced orientation (Fig. 28). In other words, the coboundary operator takes a quantity associated with the boundary of a geometric object and transfers it to the object itself. From Eq. (128) we see also that if we use the natural representation for the cochains, the coboundary operator admits the following matrix representation in terms of incidence matrices:

$$
\delta \mathbf{c}^{p}=\mathbf{D}_{p+1, p} \cdot \mathbf{c}^{p}
$$

\section{Properties of the Coboundary Operator}

The coboundary operator enjoys a number of useful properties that are a discrete version of familiar properties of differential operators (in light of our discussion about limit systems, it is more appropriate to say that the properties of the differential operators follow from those of the coboundary operator). First, as a consequence of the relationship [Eq. (90)] holding between incidence numbers [or, if you prefer, from the property $\partial\left(\partial \mathbf{c}_{p}\right)=0$, holding for any chain, and the adjointness of boundary and coboundary operators], for any cochain $\mathbf{c}^{p}$ we have

$$
\delta\left(\delta \mathbf{c}^{p}\right)=0
$$

(Hocking and Young, 1988). We will show later that the coboundary operator is the discrete counterpart (or, from another point of view, the precursor) of a differential operator $d$, which generalizes the traditional differential operators: gradient, curl, and divergence. This means in particular that the geometric 
interpretation of the action of the coboundary operator given in the previous section can be used to explain the action of these three familiar differential operators. In fact, a bit of reflection reveals that this geometric construction is implicitly used in their traditional textbook definition. In this light, for example, the identity $\delta\left(\delta \mathbf{c}^{p}\right)=0$ corresponds to the well-known identities curl $(\operatorname{grad} \varphi)=$ $\mathbf{0}$ holding for any 0 -field $\varphi$, and $\operatorname{div}(\operatorname{curl} \mathbf{A})=0$ holding for any 1 -field $\mathbf{A}$.

Another interesting observation is the fact that on a pair of dual $n$-dimensional cell complexes the following property of the coboundary operators acting at the same level and at opposite sides of the factorization diagram holds true. The coboundary transforming $p$-cochains $\mathbf{c}^{p}$ in $(p+1)$-chains of the primary complex is the adjoint, relative to a natural duality between cochains defined by suitable bilinear forms $\langle\cdot, \cdot\rangle$, of the coboundary transforming $(n-(p+1))$ cochains in $(n-p)$-cochains of the secondary complex (Mattiussi, 1997). For example, in Figure 29, this is the case of the operators acting in $\delta \mathbf{U}^{1}$ and $\delta \tilde{\Psi}^{2}$, which satisfy

$$
\left\langle\delta \mathbf{U}^{1}, \tilde{\Psi}^{2}\right\rangle=\left\langle\mathbf{U}^{1}, \delta \tilde{\Psi}^{2}\right\rangle
$$

For generic cochains this property corresponds to

$$
\left\langle\delta \mathbf{c}^{p}, \tilde{\mathbf{c}}^{n-(p+1)}\right\rangle=\left\langle\mathbf{c}^{p}, \delta \tilde{\mathbf{c}}^{n-(p+1)}\right\rangle
$$

and is expressed in terms of incidence matrices by Eq. (89). The corresponding property for differential operators is the (formal) adjointness of - grad and div, and of curl and -curl. The nondegenerate bilinear forms which put in duality the cochain spaces in Eq. (130) are, in the natural basis representation, $\left\langle\mathbf{U}^{1}, \tilde{\mathbf{Q}}^{3}\right\rangle=\sum_{i=1}^{n_{1} \tilde{n}_{3}} \mathcal{U}_{i} Q_{i}$, and $\left\langle\Phi^{2}, \tilde{\Psi}^{2}\right\rangle=\sum_{j=1}^{n_{2}=\tilde{n}_{2}} \phi_{j} \psi_{j}$ (where we have assumed that dual cells share the same index). These bilinear forms are discrete counterparts of the energy integrals for the corresponding local field quantities on which the adjointness of the differential operators is based.

In summary, by adopting the coboundary operator for the discrete representation of topological laws, and a pair of dual cell complexes as primary and secondary meshes, one automatically builds into the resulting numerical method a number of important properties of the continuous mathematical model. By so doing, contrary to what happens within many numerical methods, one is not forced to check after the discretization has been performed whether these properties are satisfied, or to enforce explicitly these properties as additional constraints in the discretization phase. Note that the prescription to write the topological equations in terms of the coboundary operator does not imply the use of some exotic mathematical entity. It means simply that you adopt the correct association of global physical quantities with oriented geometric objects, and that you write the topological equations in integral form, equating the global quantity associated with each cell with that associated with its boundary. From this point of view, when all the formal properties have been 
proved, to say that we are using the coboundary operator is simply a shortcut to signify that the sequence of steps just described is being executed.

\section{Discrete Topological Equations}

Applying the definition of the coboundary operator $\delta$, we can rewrite the topological laws of electromagnetism [Eqs. (113) through (116)] as relations between physical cochains. The steps are those leading from Eq. (116) to Eq. (126). The result is

$$
\begin{aligned}
\delta \Phi^{2} & =\mathbf{0}^{3} \\
\delta \tilde{\mathbf{Q}}^{3} & =\tilde{\mathbf{0}}^{4} \\
\delta \mathbf{U}^{1} & =\Phi^{2} \\
\delta \tilde{\Psi}^{2} & =\tilde{\mathbf{Q}}^{3}
\end{aligned}
$$

We can thus redraw the space-time classification diagram of Figure 14 in terms of cochains and coboundaries (Fig. 29). Note in the diagram of Figure 29 and in Eqs. (131) and (132) the presence of the null 3- and 4-cochain on $K$ and $\tilde{K}$, respectively.

Note also that contrary to the traditional Maxwell's equations in differential vector notation, in which positive and negative terms appear, in the cochaincoboundary notation, all signs are positive. This happens because the coboundary operator automatically takes care of the signs, by considering values on

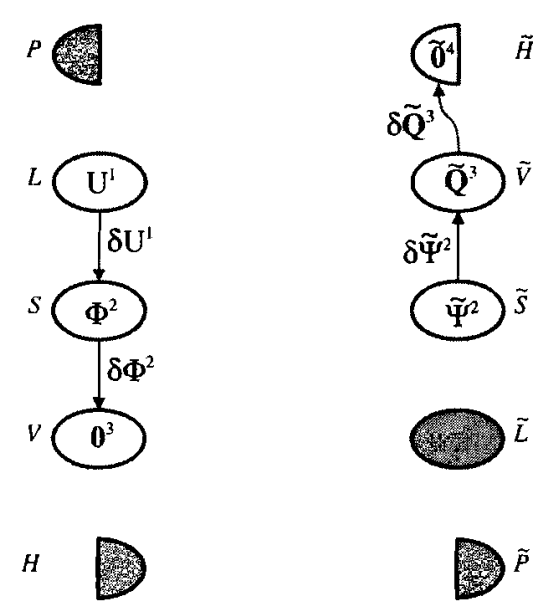

Figure 29. The Tonti classification diagram of electromagnetic physical quantities in terms of cochains, showing the topological laws in terms of the coboundary operator. 
the boundary as endowed with the induced orientation. The presence of negative signs in the traditional form of these equations is due to the fact that the default orientation of a geometric object in the mathematical definition of an operator and that in the traditional definition of a physical quantity may not agree. For example, the term $-\operatorname{grad} V$ in Eq. (11) defining the electromagnetic potential is due to the fact that in mathematics the default orientation of points is as "sinks," so that the boundary of an internally oriented line is the endpoint "minus" the starting point, whereas the traditional definition of the scalar potential $V$ implies a default orientation of points as "sources" (inherited, in fact, from the default external orientation of volumes to which electric charge is associated). Remember also, when one is considering the meaning of signs, that the quantities are associated with space-time objects and not merely with geometric objects in space.

\section{Constitutive Relations}

We said previously that the constitutive relations do not lend themselves to a natural discrete representation. Nonetheless, some kind of discrete constitutive link must be given in order to complete the discretization of the physical field problem and to arrive finally at a finite system of algebraic equations that can be subjected to the action of an algebraic solver. The task of finding the discrete constitutive link constitutes, in fact, the central problem of the discretization step. We will consider later in detail a number of possible approaches to this task, mainly inspired from existing numerical methods. For now, we shall limit ourselves to a generic analysis of the structure that this representation must possess.

Given the discrete representation of fields in terms of cochains that was presented earlier, the discrete constitutive links must be operators linking cochain spaces (usually an ordinary cochain space to a twisted one, or vice versa), ${ }^{*}$ such as

$$
\begin{aligned}
& F_{1}: C^{p}(K) \rightarrow C^{q}(\tilde{K}) \\
& F_{2}: C^{r}(\tilde{K}) \rightarrow C^{s}(K)
\end{aligned}
$$

Usually, these operators are discrete links whose structure is directly inspired by that of the local ones between field functions. For example, if we denote with

\footnotetext{
*The analysis of the factorization diagram of a great number of physical theories (Tonti, 1975) suggests that this could always be the case; that is, that every constitutive link could turn out to be a bridge between physical quantities associated with geometric objects endowed with different kinds of orientation. However, no formal proof of this conjecture seems to have been produced so far.
} 
$\tilde{\Psi}^{d}$ the electric part of the electric flux 2-cochain, and with $\Phi^{e}$ the electric part of the magnetic flux 2-cochain, the electric constitutive relation [Eq. (14)] can be given an approximate discrete representation as an operator $F_{\varepsilon}$ as follows:

$$
\tilde{\Psi}^{d}=F_{\varepsilon}\left(\Phi^{e}\right)
$$

If the required link goes from $\Phi^{e}$ to $\tilde{\Psi}^{d}$, we shall represent it as

$$
\Phi^{e}=F_{\varepsilon^{-1}}\left(\tilde{\Psi}^{d}\right)
$$

instead of as $\Phi^{e}=F_{\varepsilon}^{-1}\left(\tilde{\Psi}^{d}\right)$, to allow for discrete operators obtained by direct discretization of the local link going from $\mathbf{E}$ to $\mathbf{D}$, without limiting the choice to those obtained by inverting the link $F_{\varepsilon}$. Analogous representations hold, in both directions, for the magnetic constitutive relation [Eq. (15)] and the generalized form of Ohm's law [Eq. (16)], the discrete version of which we will write as

$$
\begin{aligned}
& \Phi^{b}=F_{\mu}\left(\tilde{\Psi}^{h}\right) \\
& \tilde{Q}^{j}=F_{\sigma}\left(\Phi^{e}\right)
\end{aligned}
$$

whereas the discrete links in the opposite direction will be written as

$$
\begin{aligned}
& \tilde{\Psi}^{h}=F_{\mu^{-1}}\left(\Phi^{b}\right) \\
& \Phi^{e}=F_{\sigma^{-1}}\left(\tilde{Q}^{j}\right)
\end{aligned}
$$

These links are usually linear operators between cochain spaces, and can, therefore, be given a natural matrix representation that we will represent, in the case of Eq. (137), as

$$
\tilde{\Psi}^{d}=\mathbf{F}_{\varepsilon} \Phi^{e}
$$

with analogous renderings for the other electromagnetic constitutive links just considered. The widespread use of a linear discrete constitutive link stems from the desire to obtain a linear system of equations by composition of the discrete constitutive operator with the coboundary operator (which is a linear operator between cochain spaces). This choice, however, does not imply that the local constitutive relation from which they derive must also be linear.* When the local constitutive relation is nonlinear, whereas the discrete constitutive equation is linear, this last link must be considered as obtained from the former by means of some kind of linearization technique within the numerical procedure.

Note that links of the kind in Eqs. (135) and (136) have an even broader scope than may seem at first sight. Consider, for example, a time-dependent problem to be solved in a domain $D$ for a time interval $I$. The space-time domain

\footnotetext{
${ }^{*}$ In fact, this will often not be the case, since, being that topological equations are always linear, nonlinearities present in the field equations are due to the constitutive terms, where they can be found when the field equations have been factorized.
} 
of the problem is the Cartesian product $D \times I$, which is decomposed by the primary and secondary cell complexes $K$ and $\tilde{K}$. Usually the decomposition is the product of a spatial decomposition $K_{s}$ of $D$ by a decomposition $K_{t}$ of $I$ (or $\tilde{K}_{s}$ and $\tilde{K}_{t}$, respectively). With this hypothesis, Eq. (135), for example, includes relations linking the values taken by $\tilde{C}^{q}$ on all $q$-cells of $\tilde{K}_{s} \times \tilde{K}_{t}$ [that is, all q-cells of $\tilde{K}_{s}$ for all time instants $\left(0\right.$-cells) of $\tilde{K}_{t}$ and all $(q-1)$-cells of $\tilde{K}_{s}$ for all time intervals (1-cells) of $\tilde{K}_{t}$ ], to the value taken by $C^{p}$ on all $p$-cells of $K_{s} \times K_{t}$ [that is, all $p$-cells of $K_{s}$ for all time instants of $K_{t}$ and all $(p-1)$-cells of $K_{s}$ for all time intervals of $K_{t}$ ]. Of course, most of the time, constitutive equations are very simple, particular cases of this general relation. A very common case, for example, with dual cell complexes and the natural dual indexing of cells, would be a diagonal operator. However, we will see later that more general cases can be usefully considered. In particular, it may happen that the actual discrete constitutive link is never explicitly determined, being obtained as a result of an algorithm, for example, an optimization procedure, taking as input a given known cochain, and giving as the result the cochain linked to the former by the constitutive relation.

The availability of a discrete representation for the constitutive relations allows the redrawing of the complete factorization diagram in discrete terms. For the case of electromagnetism, the resulting factorization diagram is depicted in Figure 30. Note the distinction between the parts of the cochains referring to time instants and those referring to time intervals, and the corresponding one for the action of the coboundary operator. This distinction is particularly useful in view of the application of the diagram to numerical methods, but remember that it applies only to space-time meshes obtained as Cartesian products of separate discretizations of the space and time domains.

\section{E. Continuous Representations}

The last few sections showed how to represent discretized domains and subdomains, fields, and topological laws in terms of cell complexes, cochains, and the coboundary operator. This can be applied straightforwardly to obtain a formal treatment of the finite volume method, which writes balance and conservation equations over subdomains which are actually simple cells, such as

$$
\int_{\tau_{2}}\left(\operatorname{curl} \mathbf{E}+\frac{\partial \mathbf{B}}{\partial t}\right)=0
$$

and

$$
\int_{\tau_{3}} \operatorname{div} \mathbf{B}=0
$$



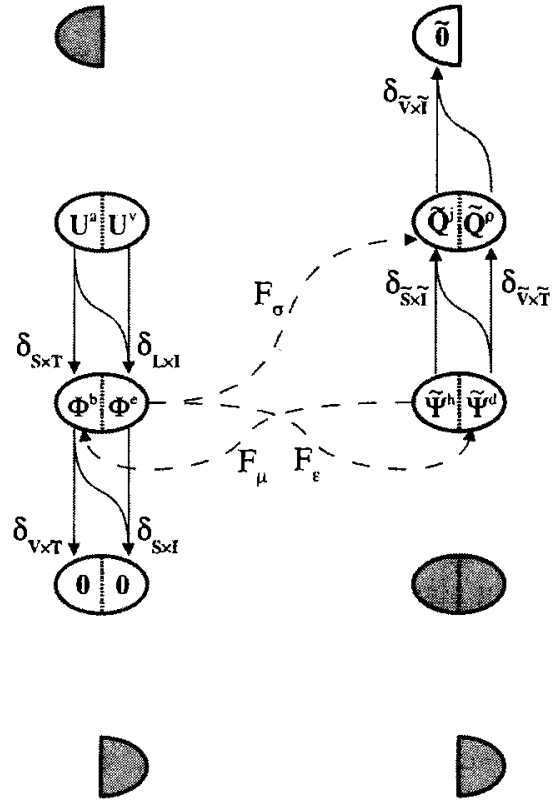

FIGURE 30. The Tonti discrete factorization diagram of electromagnetism, with the fields represented in terms of cochains, the topological laws in terms of the coboundary operator, and the constitutive links as operators between cochain spaces.

which, after application of the integral theorems of vector calculus, become

$$
\begin{aligned}
\int_{\partial \tau_{2}} \mathbf{E}+\int_{\tau_{2}} \frac{\partial \mathbf{B}}{\partial t} & =0 \\
\int_{\partial \tau_{3}} \mathbf{B} & =0
\end{aligned}
$$

The case of finite element methods, however, does not fit equally well in a representation built on these purely discrete concepts. For example, a weighted residual formulation of Eqs. (144) and (145) is

$$
\begin{aligned}
\int_{\tau_{3}} \mathbf{w} \cdot\left(\operatorname{curl} \mathbf{E}+\frac{\partial \mathbf{B}}{\partial t}\right) & =0 \\
\int_{\tau_{3}} w \operatorname{div} \mathbf{B} & =0
\end{aligned}
$$


where $\mathbf{w}$ is a vector-valued weight function, $w$ is a scalar one, and $\tau_{3}$ is a regular-three-dimensional domain. After integration by parts, Eqs. (148) and (149) become

$$
\begin{array}{r}
\int_{\tau_{3}} \operatorname{curl} \mathbf{w} \cdot \mathbf{E}-\int_{\partial \tau_{3}} \mathbf{w} \times \mathbf{E}+\int_{\tau_{3}} \mathbf{w} \cdot \frac{\partial \mathbf{B}}{\partial t}=0 \\
\int_{\tau_{3}} \operatorname{grad} w \cdot \mathbf{B}-\int_{\partial \tau_{3}} w \mathbf{B}=0
\end{array}
$$

which do not convey the immediate geometric meaning of Eqs. (146) and (147).

The weighted residual technique just shown in action, taken as representative of the strategy adopted by the finite element methods, permits nonetheless a geometric interpretation that parallels that of the finite volume methods. To display this analogy, we will introduce some continuous concepts that correspond to the discrete ones introduced so far in the representation of geometry, fields, and topological laws (Table 1). Note that the aim of the present section is not the construction of an abstract correspondence between discrete

TABLE 1

\begin{tabular}{|c|c|c|c|}
\hline \multicolumn{2}{|r|}{ Discrete } & \multicolumn{2}{|c|}{ Continuous } \\
\hline$p$-cell & $\tau_{p}$ & $D_{p}$ & $p$-dimensional domain \\
\hline Boundary of a $p$-cell & $\partial \tau_{p}$ & $\partial D_{p}$ & Boundary of a $p$-dimensional domain \\
\hline$p$-chain & $\mathbf{c}_{p}$ & $\mathbf{w}_{p}$ & Weighted $p$-domain \\
\hline$p$-skeleton of a cell complex & $K_{p}$ & $W_{p}$ & Collection of weighted $p$-domains \\
\hline$p$-cochain & $c_{p}$ & $\omega^{p}$ & $p$-form \\
\hline Pairing of $p$-chain and $p$-cochain & $\left(\mathbf{c}_{p}, \mathbf{c}^{p}\right)$ & $\int_{\mathbf{w}_{p}} \omega^{p}$ & Weighted $p$-integral of a $p$-form \\
\hline Coboundary operator & $\delta$ & $d$ & Exterior differential operator \\
\hline $\begin{array}{l}\text { Discrete generalized Stokes's } \\
\qquad\left(\tau_{p+1}, \delta \mathbf{c}^{p}\right)=(\partial \tau\end{array}$ & $\begin{array}{l}\text { theorem } \\
\left.\tau_{p+1}, c^{p}\right)\end{array}$ & \multicolumn{2}{|c|}{$\begin{array}{l}\text { Continuous generalized Stokes's theorem } \\
\int_{D_{p+1}} d \omega^{p}=\int_{\partial D_{p+1}} \omega^{p}\end{array}$} \\
\hline $\begin{array}{r}\text { Discrete Green's } \\
(\text { summation } \mathrm{t} \\
\left(\mathbf{c}_{p+1}, \delta \mathbf{c}^{p}\right)=(\partial \mathbf{c}\end{array}$ & $\begin{array}{l}\text { formula } \\
\text { by parts) } \\
\left.\mathbf{c}_{p+1}, \mathbf{c}^{p}\right)\end{array}$ & \multicolumn{2}{|c|}{$\begin{array}{l}\text { Continuous Green's formula } \\
\text { (integration by parts) } \\
\int_{w_{p+1}} d \omega^{p}=\int_{\partial w_{p+1}} \omega^{p}\end{array}$} \\
\hline $\begin{array}{r}\text { Discrete topological } \\
\left(\partial \mathbf{c}_{p+1}, \mathbf{a}^{p}\right)=\left(\mathbf{c}_{p+1}, \mathbf{b}^{p+1}\right) \\
(\text { stror } \\
\delta \mathbf{a}^{p}\end{array}$ & $\begin{array}{r}\text { equation } \\
\text { ak form) } \\
\forall \mathbf{c}_{p+1} \\
\text { ng form) } \\
=\mathbf{b}^{p+1}\end{array}$ & \multicolumn{2}{|c|}{$\begin{array}{l}\int_{\partial \mathbf{w}_{p+1}} \alpha^{p}=\int_{\mathbf{w}_{p+1}} \beta^{p+1} \quad \forall \mathbf{w}_{p+1} \\
\text { (strong form) }\end{array}$} \\
\hline
\end{tabular}

TABLe of Correspondences between Discrete and Continuous Concepts 
and continuous concepts. The inspiration for the parallelism comes from (and is instrumental to the application to) numerical methods. The actual formal justification of a particular correspondence-that is, the proof that in the limit the discrete concept goes into the continuous one-may be very difficult, or even impossible. In this case we will simply be confident in the heuristic interpretative value of the correspondence thus built.

To parallel the presentation of discrete representations made so far, we should ideally deal first with continuous models for the domain geometry. It turns out, however, that a preliminary discussion of continuous field representations paralleling cochains is preferable. The only concept that we must suppose available is that of $n$-dimensional domain of integration $D_{n}$ contained in the domain $D$, which can be considered as an $n$-dimensional differentiable manifold and usually is merely a regular subdomain of $\mathbb{R}^{n}$.

\section{Differential Forms}

Given expressions such as Eqs. (150) and (151), if we want to find a geometric interpretation for the weighted residual methods, it is clear that we will have to deal with integrals. Strictly speaking, the "thing" that is subjected to integration on oriented $p$-dimensional domains is a $p$-dimensional differential form (usually called simply a p-form) (Deschamps, 1981; Warnick et al., 1997). If the domain is internally oriented, we speak of an ordinary $p$-form, which we denote by $\omega^{p}$; otherwise, the $p$-form is called twisted and is denoted by $\tilde{\omega}^{p}$ (Burke, 1985). We said previously that $p$-cochains associate values with $p$-cells and that this association is additive on the sum of cells. This is true also for the integration of $p$-forms on $p$-dimensional domains. In fact, a $p$-form on a cellulizable domain $D$ gives rise to a $p$-cochain $\mathbf{c}^{p}\left(K_{\beta}\right)$ on each cell complex $K_{\beta}$ which subdivides $D$, since it associates with each cell $\tau_{p}^{i}$ a value $c^{i}$, where

$$
c^{i}=\int_{\tau_{p}^{i}} \omega^{p}
$$

Note how this parallels the original definition of the action of a cochain, as associating with the cell the value

$$
c^{i}=\left(\tau_{p}^{i}, \mathbf{c}^{p}\right)
$$

To emphasize this parallelism. Bamberg and Sternberg (1988) call $p$-forms also incipient p-cochains. In fact, we can think of the $p$-form $\omega^{n}$ as a particular representation of an element $\left\{\mathbf{c}^{p}\left(K_{\alpha}\right)\right\}$ of the inverse limit group $C^{p}\left(K_{\infty}\right)$. The particular cochain $\mathbf{c}^{p}\left(K_{\beta}\right)$ is then the projection of $\omega^{p}$ on the cell complex $K_{\beta}$.

Given the correspondence of Eq. (152) with Eq. (153), we can ask what corresponds to the more general pairing of chains and cochains $\left(\mathbf{c}_{p}, \mathbf{c}^{p}\right)$. At 
first sight, we could consider an integral on a chain, using the definition

$$
\int_{\mathbf{c}_{p}} \omega^{p}=\int_{\Sigma_{i} w_{i} \tau_{p}^{i}} \omega^{p} \stackrel{\text { def }}{=} \sum_{i} w_{i} \int_{\tau_{p}^{i}} \omega^{p}
$$

This is, in fact, the most general definition of integration on manifolds usually given in textbooks (Choquet-Bruhat and DeWitt-Morette, 1977; Flanders, 1989). A bit of reflection, however, shows that this extension of the concept of integral, from $p$-dimensional domains to $p$-chains, does not fulfill our needs, since the integrals appearing in weighted residual expressions such as Eqs. (148) and (149), in the language of differential forms, correspond to

$$
\int_{\tau_{p}} w \omega^{p}
$$

where $w$ is a weight function defined on $\tau^{p}$. To find a way out of this impasse, we could think of Eq. (155) as derived from the right-hand side of Eq. (154) by a limit process that considers chains built on finer and finer meshes, or, alternatively, we could reconsider the way a Riemann integral is evaluated by partitioning the integration domain. We will pursue both lines of thought in the next subsection.

One could at this point argue that finite element methods do not actually use formulations based on differential forms, since expressions such as Eqs. (148) and (149) have a scalar or vector field function in place of the differential form $\omega^{p}$. This is, however, merely an unfortunate consequence of the historical development of vector calculus as applied to physics. The reduction of what are actually differential $p$-forms to only scalars and vectors in fact makes things more difficult to understand physically and to represent geometrically. One has only to browse through books such as Burke (1985), Schouten (1989), and Misner et al. (1970), and papers such as Warnick et al. (1977), with their fascinating geometric representations of forms and integration, to convince ourselves of this fact.

\section{Weighted Integrals}

Although the idea behind the operation of actually integrating a $p$-form on a differentiable manifold is intuitive, one must face some technical problems. For example, to define an analogy of Riemann integration, one must represent the integration domains and their partitions. This problem is usually circumvented, thanks to the presence of a collection of maps-the so-called coordinate maps - of a collection of open sets of the manifold where the integration takes place, onto open subsets of a suitable Euclidean space (Bishop and Goldberg, 1980; de Rham, 1960). This allows the definition of the pullback of a form 
from the manifold to the Euclidean space (Burke, 1985), where the familiar machinery of Riemann integration is usually invoked. Justification of this step often involves the idea that the differential form subject to integration in the manifold becomes, after pullback, an ordinary scalar function in Euclidean space, while the integration domain within the manifold becomes a Euclidean domain, which can be easily partitioned into pieces of known extension. But how does it happen that a differential form within the manifold becomes an ordinary function in the Euclidean space? There is, in fact, a step that is usually passed over silently in forming the Riemann sums that define the integral. This step is the pairing of the pulled-back form with a collection of multivectors, which represent the parallelepipeds partitioning the domain in the Riemann integration procedure. This concept of multivector just introduced requires a brief digression.

The calculus of differential forms is built on the algebra of forms, which defines forms as linear functions defined on spaces of multivectors. To this end one starts from a vector space and defines first the concept of $p$-vector $\mathbf{v}_{p}$ (Birss, 1980). You can think of a $p$-vector as defining a $p$-dimensional oriented domain within an affine space (Tonti, 1975). Thus, a 0 -vector $v_{0}$ is a scalar; a 1 -vector $\mathbf{v}_{1}$ is the familiar vector and defines an oriented segment along a line; a 2-vector $\mathbf{v}_{2}$, or bivector, defines an oriented surface on a plane; a 3-vector $\mathbf{v}_{3}$, or trivector, defines an oriented volume; and so on, up to the maximum dimension allowed by the affine space.* Paralleling the distinction between internal and external orientation for geometric objects, ordinary multivectors, corresponding to internally oriented geometric objects, and twisted multivectors, corresponding to externally oriented geometric objects, can be defined (Burke, 1985; Fig. 31). Note that $p$-vectors constitute in turn a vector space, and that we can define the extension of a $p$-vector without recourse to metric concepts.

Given the concept of multivector, one can define an algebraic $p$-form as a linear function on the space of $p$-vectors, with values in an algebraic field. From this definition it follows that the pairing of a $p$-vector $\mathbf{v}_{p}$ and a $p$-form $\omega^{p}$ gives a value, exactly like the pairing of a chain and a cochain (see Misner et al., 1970, and Warnick et al., 1997, for fascinating geometric illustrations of this pairing). This analogy suggests the following representation of the pairing:

$$
\left(\mathbf{v}_{p}, \omega^{p}\right)
$$

*Actually, the situation can be more complex, as a result of the fact that, for example, in four-dimensional space a generic multivector can be compound (Schouten, 1989; Tonti, 1975). However, compound multivectors are not required to represent common geometric objects (Birss, 1980 ). 


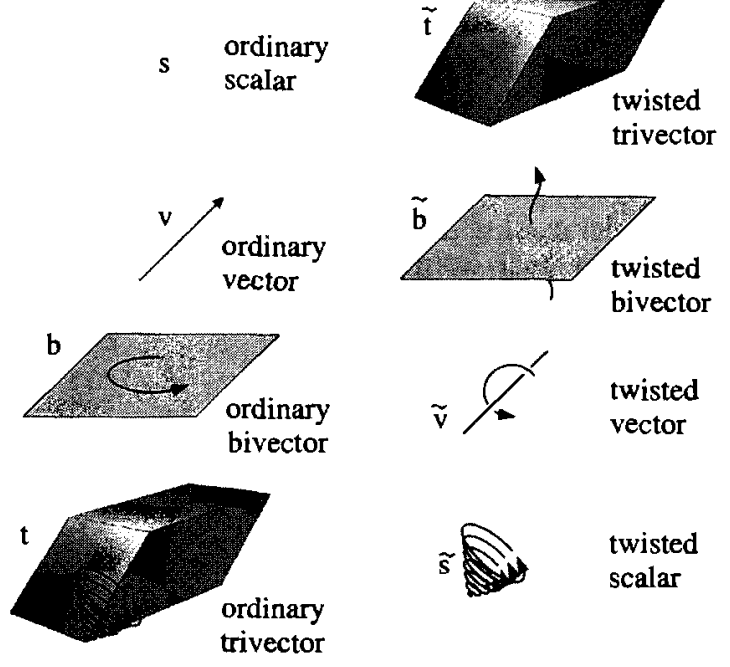

FIGURE 31. Ordinary and twisted multivectors correspond to internally and externally oriented geometric objects, respectively. Here the case of a three-dimensional vector space is represented.

Given these steps, so that the Riemann integral of a $p$-form in a Euclidean space can be defined, the integration domain $D_{p}$ is subdivided into a collection $V_{p}$ of $p$-dimensional parallelepipeds, which can be thought of as $p$-vectors $\mathbf{v}_{p}^{i}$ (Dezin, 1995). The corresponding Riemann sum is, therefore,

$$
S=\sum_{i}\left(\mathbf{v}_{p}^{i}, \omega^{p}\right)
$$

In the sequence of domain partitions considered in the Riemann integration process, the maximum $p$-vector extension tends to zero, and the corresponding limit, existing for suitable regularity conditions, is the integral of the form

$$
\int_{D_{p}} \omega^{p}
$$

We see, therefore, that in building a Riemann sum we actually pair the differential form with a chain composed by the collection $V_{p}$ of multivectors, which partition the domain. An obvious extension paralleling that which assigns real weights to cells in the formation of chains consists of using collections of weighted multivectors. This can be done by assigning a scalar weight function $w$ on the integration domain. We can then define the new Riemann sums as 
follows:

$$
S=\sum_{i}\left(w\left(\xi^{i}\right) \mathbf{v}_{p}^{i}, \omega^{p}\right)
$$

where $\xi^{i}$ is a point belonging to the $p$-vector $\mathbf{v}_{p}^{i}$ of the domain decomposition. Under the usual regularity conditions, the value of the limit of the sequence of Riemann sums with decreasing maximum extension of the multivectors does not depend on the actual position of $\xi^{i}$ in $\mathbf{v}_{p}^{i}$. This limit is the weighted integral and can be denoted by

$$
\int_{w_{p}} \omega^{p}
$$

This symbolism emphasizes the fact that the function $w$ weights the integration domain and is not an ordinary function that multiplies the form. This can be thought of as saying that integrals of the kind [Eq. (159)] [which includes as particular cases those appearing in Eqs. (148) through (151)] must be considered Stieltjes integrals and not ordinary integrals of the products of functions (Lebesgue, 1973).

If the integral is defined on a regular $p$-dimensional domain that lies in a manifold, we can, as anticipated, pull back the form and apply the procedure just described for the integration in Euclidean spaces. It is, however, instructive to consider the possibility of going the other way; that is, to push forward the multivectors that partition the Euclidean domain (Burke, 1985). This can be thought of as providing a decomposition of the integration domain in the manifold. There are actually some technical difficulties, since the vectors thus pushed forward do not "belong" to the manifold but to its tangent space (Burke, 1985). We can circumvent this problem, saving the heuristic value of the idea, by thinking, for example, of manifolds which are subsets of a suitable Euclidean space, so that tangent multivectors are actually tangent parallelepipeds that approximate the true image of the Euclidean parallelepiped on the manifold (e.g., see Figs. 8.24 and 8.25 and the related discussion in Bamberg and Sternberg, 1988). To this "decomposition" of the integration domain in the manifold apply all the considerations just made for Riemann sums and weighted integrals, and in particular the role of the function $w$ in Eq. (159) in weighting the "cells" of the decomposition of the domain considered in a Riemann sum; that is, in giving a collection of finer and finer chains, which can be thought of as the continuous counterpart of a chain.

\section{Differential Operators}

To develop further the correspondence between differential forms and cochains, let us consider the action of the coboundary operator. The definition [Eq. (122)] 
of the coboundary operator on the cochains of a cell complex $K$ was given in order to allow the transition from a topological equation in the form

$$
\left\langle\partial \tau_{p+1}, \mathbf{a}^{p}\right\rangle=\left\langle\tau_{p+1}, \mathbf{b}^{p+1}\right\rangle \quad \forall \tau_{p+1} \in K
$$

to the following direct relation between cochains:

$$
\delta \mathbf{a}^{p}=\mathbf{b}^{p+1}
$$

We can mimic the definition [Eq. (122)] of the coboundary operator in the terminology of differential forms. We obtain

$$
\int_{D_{p+1}} d \omega^{p} \stackrel{\text { def }}{=} \int_{\partial D_{p+1}} \omega^{p} \quad \forall D_{p+1} \subset D
$$

where $d$ is an operator transforming $p$-forms into $(p+1)$-forms. This operator is called the exterior differential and inherits the property of the coboundary operator of allowing the transition from Eq. (160) to Eq. (161), by transforming a topological equation given in integral form, such as

$$
\int_{\partial D_{p+1}} \alpha^{p}=\int_{D_{p+1}} \beta^{p+1} \quad \forall D_{p+1} \subset D
$$

into

$$
d \alpha^{p}=\beta^{p+1}
$$

Note that usually the exterior differential is defined in terms of derivatives of the form's components, whereas Eq. (162) constitutes an intrinsic definition (Isham, 1989) which, as emphasized by one of the creators of the calculus of forms, ${ }^{*}$ does not require the existence of the derivatives of the form's components. The generic operator $d$ defined by Eq. (162) combines in a unique operator the action of the familiar differential operators gradient, curl, and divergence, which can also be given an intrinsic definition. Remembering that we call $p$-field that which corresponds to a quantity associated with $p$-dimensional geometric objects, we can give the following definitions. The gradient operator acts on 0 -fields and gives 1-fields, which satisfy

$$
\int_{D_{1}} \operatorname{grad} \varphi \stackrel{\text { def }}{=} \int_{\partial D_{1}} \varphi \quad \forall D_{1} \subset D
$$

The curl operator acts on 1-fields and produces 2-fields according to

$$
\int_{D_{2}} \operatorname{curl} \mathbf{A} \stackrel{\text { def }}{=} \int_{\partial D_{2}} \mathbf{A} \quad \forall D_{2} \subset D
$$

*"On conÇoit donc la possibilité de définir la dérivation extérieure comme une opération autonome, indépendante de la dérivation classique" (Cartan, 1922) p. 69. 
Likewise, the divergence operator acts on 2-fields and gives 3-fields satisfying

$$
\int_{D_{3}} \operatorname{div} \mathbf{B} \stackrel{\text { def }}{=} \int_{\partial D_{3}} \mathbf{B} \quad \forall D_{3} \subset D
$$

It is worth noting once more that the property $\delta \delta=0$ of the coboundary operator is reflected in the property $d d=0$ of the exterior differential operator, which in turn corresponds to curl grad $=0$ and div curl $=0$ in vector calculus notation.

Given its properties, the exterior differential $d$ appears as the equivalent of the limit operator $\delta_{\infty}$ defined at the end of the section on limit systems (Section III.B.2). It is no wonder then that its definition can be based on global concepts. Of course, given the additivity of global physical quantities, and the telescoping property following from the opposite orientation induced on the common boundary by adjacent, coherently oriented domains, if the definition [Eq. (162)] of $d$ (and those [Eqs. (165) through (167)] of the traditional differential operators of the vector calculus) is enforced in the small, it holds for every geometric object. This is why in textbook expositions the definitions [Eqs. (165) through (167)] are applied to infinitesimal one-, two-, and threedimensional rectangles, to derive the definition in local terms of the operators. This gives the familiar expressions in terms of derivatives, but our approach shows that these operators have a more general significance.

The three-to-one relation of the differential operators of vector analysis with the exterior differential of forms stems from the already signaled limitations of representation in terms of vectors alone, which hide the true " $p$-nature" of $p$-fields. Our treatment reveals, for example, that an expression of the kind

$$
\operatorname{curl}(\operatorname{curl} \mathbf{A})
$$

is meaningless as such, for the 2-field produced by the first application of the operator cannot be operated onto by the second operator. The actual expression should, therefore, be actually something such as

$$
\operatorname{curl}(k(\operatorname{curl} \mathbf{A}))
$$

where the intermediate operator $k$ represents an operator, for example, a constitutive link, which transforms a 2-field into a 1-field (which, if $k$ is a constitutive operator, is usually endowed with a different kind of orientation with respect to $\mathbf{A}$ ).

Using differential forms and the exterior differential one can rewrite in a compact way the equations of electromagnetism. One starts by grouping everything related to a given space-time geometric object in a unique differential form. Thus, remembering the classification of electromagnetic quantities already defined, one has an ordinary 2 -form-the electromagnetic 2-form $F^{2}$, 
which "groups" $\mathbf{E}$ and $\mathbf{B}$, or, better, the local counterparts of $\phi^{e}$ and $\phi^{\mathrm{b}}$-and a twisted 3-form-the charge-current 3-form $\tilde{J}^{3}$, grouping $\mathbf{J}$ and $\rho$. The local conservation of magnetic flux is expressed by $d F^{2}=0^{3}$, and that of electric charge by $d \tilde{J}^{3}=\tilde{0}^{4}$. The charge-current potentials $\mathbf{H}$ and $\mathbf{D}$ go into a twisted 2-form $\tilde{G}^{2}$, related to $\tilde{J}^{3}$ by $d \tilde{G}^{2}=\tilde{J}^{3}$, whereas the electromagnetic potentials go into an ordinary 1-form $A^{1}$ satisfying $d A^{1}=F^{2}$. The constitutive relations are expressed by a mapping between differential forms; for example, the electric and magnetic constitutive relations are expressed by $\tilde{G}^{2}=\chi\left(F^{2}\right)$, where $\chi$ is a generic operator from the space of ordinary 2 -forms to that of twisted 2 -forms (as detailed subsequently, often erroneously identified with the Hodge star operator). The construction of the corresponding factorization diagram in terms of differential forms is straightforward.

\section{Spread Cells}

Let us now go back to weighted integrals and combine their properties with those of the newly defined differential operator. We have at last with $\int_{w_{p}} \omega^{p}$ an expression fully correspondent, in a continuous setting, to the chain-cochain pairing $\left(\mathbf{c}_{p}, \mathbf{c}^{p}\right)$. This is a bilinear pairing with respect to which the boundary and coboundary operators are mutually adjoint, satisfying the relation $\left(\mathbf{c}_{p+1}, \delta \mathbf{c}^{p}\right)=\left(\partial \mathbf{c}_{p+1}, \mathbf{c}^{p}\right)$. In a similar way we can define the adjoint of the exterior differential as the boundary of the weighted domain. Formally this produces

$$
\int_{w_{p+1}} d \omega^{p}=\int_{\partial w_{p+1}} \omega^{p}
$$

and can be given an explicit expression in terms of differential forms (Bamberg and Sternberg, 1988). Since we are interested in its application within the weighted residual method, to interpret formulas such as Eqs. (148) and (149), let us see instead how this appears in the familiar language of vector calculus (remember that the exterior differential operator $d$ corresponds to the gradient, curl, or divergence operators, depending on the type of field under consideration). Integrating by parts the expression that corresponds to the left side of Eq. (170) when $d$ is the divergence operator, we have

$$
\int_{\tau_{3}} w \operatorname{div} \mathbf{D}=\int_{\partial \tau_{3}} w \mathbf{D}-\int_{\tau_{3}} \operatorname{grad} w \cdot \mathbf{D}
$$

where the 3-cell $\tau_{3}$ can be taken as the support of the weight function $w .^{*}$ The right side of Eq. (171) can be considered to correspond to that of Eq. (170);

*The support of a function is the closure of the set of points where it does not vanish (Bossavit, 1998a). 
that is, the expression for the "boundary" of a weighted three-dimensional geometric object. In other words, we can give the following formal definition (Mattiussi. 1997):

$$
\int_{\partial\left(w \tau_{3}\right)} \mathbf{D} \stackrel{\text { def }}{=} \int_{\partial \tau_{3}} w \mathbf{D}-\int_{\tau_{3}} \operatorname{grad} w \cdot \mathbf{D}
$$

where with $w \tau_{3}$ we represent a weighted 3-cell. Note that, as anticipated while speaking of the boundary of chains, this "boundary" includes actually an integral on the whole 3-cell $\tau_{3}$, and not only on $\partial \tau_{3}$, except in the particular case of a weight function which is constant on its support.

We can, therefore, give the following geometric interpretation to the corresponding weighted residual formulas. The weight function $w$ defines the continuous counterpart of a chain. We can think of it as a "spread" or "smeared out" cell (Mattiussi, 1997), to be compared with the "crisp" cells considered so far, which can be characterized by a weight function that is constant on its support (Oñate and Idelsohn, 1992; Fig. 32). When an expression such as

$$
\int_{\tau_{3}} w \operatorname{div} \mathbf{D}=\int_{\tau_{3}} w \rho
$$

is written within a finite element formulation of an electromagnetic problem, and the left-hand side is integrated by parts to get

$$
\int_{\partial \tau_{3}} w \mathbf{D}-\int_{\tau_{3}} \operatorname{grad} w \cdot \mathbf{D}=\int_{\tau_{3}} w \rho
$$

we can consider this last formula as the expression of the balance between the electric charge associated with the corresponding spread cell and the electric

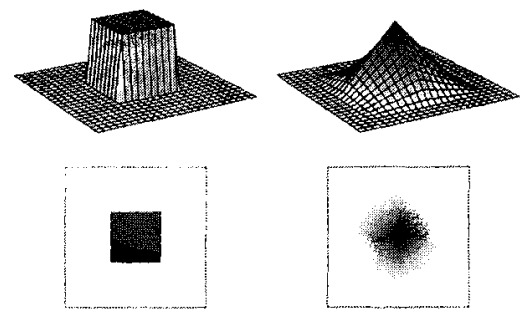

FIGURE 32. Weight functions which are constant on their support define crisp cells (left). Generic weight functions define instead the continuous counterpart of a chain that can be thought of as a spread cell (right). 
flux associated with the boundary of that spread cell; that is,

$$
\int_{\partial\left(w_{\tau_{3}}\right)} \mathbf{D}=\int_{u / \tau_{3}} \rho
$$

If the weight function is proportional to the characteristic function of a cell, that is, it is constant on the cell and is zero outside, the second term of the left-hand side of Eq. (174) vanishes, and the finite element method corresponds to the finite volume method (Fletcher, 1984; Oñate and Idelsohn, 1992). Otherwise, the finite collection $W=\left\{\mathbf{w}_{p}^{i}\right\}$ of weight functions used within a weighted residual finite element formulation can be thought of as defining a continuous counterpart of a cell complex, composed by spread cells. Of course, these spread cells usually overlap, whereas the $p$-cells of a cell complex meet at most on lower-dimensional cells. However, if the weight function constitutes a partition of unity in the domain (Belytschko et al., 1996), something of the spirit that dictated that request for cell complexes remains valid, since the sum of the physical quantities associated with the spread cells of $W$ equals the amount of that quantity associated with the entire domain. Note that the role of integration by parts, or, if you prefer, of Green's formulas, is interpreted geometrically as defining implicitly the boundary of a spread cell. For this reason, the corresponding discrete formula [Eq. (123)] can be called the discrete Green's formula or the summation by parts formula. It is worth emphasizing that this summation by parts formula, contrary to those used in the context of compact finite difference methods (Bodenmann, 1995; Strand, 1994; Lele, 1992), is based on topological concepts only and does not require the preliminary definition of an inner product. Moreover, the summation by parts formula [Eq. (123)] is automatically satisfied by adopting a discretization based on cell complexes, chains, cochains, and the corresponding operators, and, therefore, need not be imposed explicitly on the discrete operators which substitute the differential ones.

The relation corresponding to Eq. (171) for the case of two-dimensional domains is

$$
\int_{\tau_{2}} w \operatorname{curl} \mathbf{E}=\int_{\partial \tau_{2}} w \mathbf{E}-\int_{\tau_{2}} \operatorname{grad} w \times \mathbf{E}
$$

where $\mathbf{E}$ is a generic 2-field. This leads to the following formal definition

$$
\int_{\partial\left(w \tau_{2}\right)} \mathbf{E} \stackrel{\text { def }}{=} \int_{\partial \tau_{2}} w \mathbf{E}-\int_{\tau_{2}} \operatorname{grad} w \times \mathbf{E}
$$

for the boundary of a spread 2-cell, to be used, for example, to enforce the 
following relation:

$$
\int_{\partial\left(w \tau_{2}\right)} \mathbf{E}+\int_{w \tau_{2}} \frac{\partial \mathbf{B}}{\partial t}=0
$$

An expression such as Eq. (177) would, however, find application within the finite element formulation of a three-dimensional problem, only if a peculiar kind of discretization were defined for the domain that mixed discrete and continuous concepts. An example of such a discretization would be a collection of weight functions defined on the 2-cells of a cell complex that subdivides the three-dimensional domain of the problem.

For weighted one-dimensional domains the following definition would apply:

$$
\int_{\partial\left(w \tau_{1}\right)} \varphi \stackrel{\text { def }}{=} \int_{\partial \tau_{1}} w \varphi-\int_{\tau_{1}} \operatorname{grad} w \varphi
$$

As before, its use within a numerical method requires a mesh including a collection of spread 1-cells distributed within the domain of the problem.

These kinds of formulations, mixing continuous and discrete concepts in the construction of the meshes, are not currently used in the numerical practice. Instead in an $n$-dimensional domain, only $n$-dimensional weighted integrals are considered, such as the first term on the left side of Eq. (148) in place of that of Eq. (176). In this case, one can still think that the vector $\mathbf{w}(\xi)$, where $\xi$ is a point within the support of the weight function $\mathbf{w}$, defines locally a weight for bivectors orthogonal to $w(\xi)$ (if the entities subjected to weighted integration are 2-fields) or vectors parallel to $\mathbf{w}(\xi)$ (if the entities are 1-fields). Thus some remnant of the geometric meaning of the weighted residual equation is still present in these formulations. Note that if the well-known integrability conditions hold, the support of $\mathbf{w}$ can be thought of as sliced into a collection of spread cells. ${ }^{*}$ For example, irrotational weight functions $\mathbf{w}(\xi)$ define a collection of surfaces orthogonal to the field $\mathbf{w}$, whereas solenoidal weight functions define a collection of lines along it. Note that these cases correspond to the absence, in the expression for the boundary of the corresponding weighted domain, of terms that are integrated on the interior of the support of the weight function (this is the continuous counterpart of the presence of "interior" cells in the boundary of a generic chain).

\section{Weak Form of Topological Laws}

We call the strong solution of a physical field problem that which satisfies its mathematical model in terms of partial integrodifferential equations

\footnotetext{
*The collection of domains supporting the cells can be a foliation, or, in the presence of singularities, a stratification (Abraham et al., 1988).
} 
supplemented by a set of boundary conditions (Oden, 1973). Correspondingly, this mathematical model is called the strong formulation of the problem. Let us borrow this name and apply it to the differential formulation of topological laws. Hence, we shall call Eqs. (7) through (13) the strong form of the topological laws of electromagnetism, and Eqs. (70) and (71) the strong form of those of heat transfer. In the language of differential forms, these equations can all be rewritten as follows:

$$
d \alpha^{p}=\beta^{p+1}
$$

where $\alpha^{p}$ and $\beta^{p+1}$ are suitable differential forms representing the fields involved, and $d$ is the exterior differential operator. We said that from the point of view of inverse limit systems, the operator $d$ can be interpreted as the operator $\delta_{\infty}$-that is, a collection of coboundary operators acting between the projections on the directed set $\mathcal{K}$ of all the cell complexes which subdivide the domain of the problem - of the fields represented by $\alpha^{p}$ and $\beta^{p+1}$. Therefore, a strong topological statement such as Eq. (180) can be interpreted as the collection of all the corresponding discrete topological statements (in terms of cochains and coboundary). Thus, Eq. (180) is equivalent to

$$
\delta \mathbf{A}^{p}(K)=\mathbf{B}^{p+1}(K) \quad \forall K \in \mathcal{K}
$$

where $\mathbf{A}^{p}(K)$ and $\mathbf{B}^{p+1}(K)$ are the cochains resulting from the projection of $\alpha^{p}$ and $\beta^{p+1}$ on the cell complex $K$. Seen in this light, the weak and strong formulations of topological laws are different only in our considering the collection of topological statements as an assembly, or as a single entity. This approach applies also to the case of spread cells; that is, to the enforcement of topological laws in terms of weighted integrals discussed previously. Of course, the collection of spread cells must be wide enough so that practically all the conceivable topological statements will be enforced. Thus, when we select a suitable space $\mathcal{W}$ of weight functions, a statement such as

$$
\int_{\partial w} \alpha^{p}=\int_{w} \beta^{p+1} \quad \forall w \in \mathcal{W}
$$

(where, with some notational abuse, we have identified the weight function with the weighted domain of integration) "leaves nothing to be desired" (Bossavit, 1998b) from the point of view of the enforcement of the topological law expressed by Eq. (180). In fact, it turns out that Eq. (182) is a more comprehensive statement than Eq. (180), since it is not disturbed by the presence of discontinuities in the field, which require instead the enforcement of separate interface conditions when the strong formulation is adopted. Inspired by the language of functional analysis, we can call Eq. (182) the weak formulation of the topological law.

The equivalence between weak and strong formulations of topological laws no longer holds if we consider one, or at most a few, cell complexes instead 
of the complete collection of cell complexes which subdivide the domain. This is the case for numerical methods in which only one mesh for each kind of orientation is built in the domain, and consequently only the topological statements corresponding to the actual meshes are enforced. Of course, since we are considering in the domain only the physical quantities associated with the geometric objects of the meshes, we cannot hope to enforce a wider set of topological equations than those which involve these quantities (which, however, are enforced exactly). In particular, if we build a field function defined on the whole domain, starting from the finite collection of global quantities defined on the complex and satisfying on it the corresponding topological law, we can expect topological prescriptions not included in those enforced to be violated (Bossavit, 1998a).

\section{METHODS}

\section{A. The Reference Discretization Strategy}

We have at this point all the elements to ascertain whether or not a discretization strategy complies with the tenets derived from an analysis of the mathematical structure of physical field theories. To provide a framework for the development of new methods that satisfy these requirements, and to facilitate the comparison of these principles with those adopted by a number of popular numerical methods, we will now describe a reference discretization strategy directly based on the ideas developed so far. Note that this reference strategy does not qualify as a complete numerical method since the discretization of the constitutive relations is described in generic terms only. However, it will be clear that a whole class of methods complying with the analysis discussed so far can be obtained by combining the elements of the reference strategy. In fact, and this is one of the central points of this article, the reference discretization strategy is intended as a template to be used for the systematic construction of numerical methods for field problems complying with the structure of the underlying physical theory. The only prerequisite for the application of this template is the determination of the factorization diagram for the problem for which a numerical method is sought. Seen in this more limited practical perspective, all the discussion so far can be interpreted as an introduction to the language and tools that allow the correct execution of this preliminary step, the reference discretization strategy in itself being reformulable in traditional mathematical terms without the need to refer to the ideas of algebraic topology.

The reference discretization strategy is presented here for the case of timedependent electromagnetic problems, since at this point we know the structure of the factorization diagram for this theory. Moreover, although the analysis 
introduced in the present work applies to static problems as well, we know that it is in space-time that it comes to full fruition. As a consequence of this choice of the problem for the reference strategy, the comparison that will follow will consider mostly time-domain methods, be they finite difference, finite volume, or finite element methods. In summary, we consider, as the subject of the discretization, within a bounded space-time domain, a problem constituted by Maxwell's Eqs. (7), (8), (12), and (13) [or any of the integral formulations derived from them within the present work; in particular, the fully discrete form represented by Eqs. (56), (57), (61), and (62)], supplemented by a set of constitutive relations [e.g., Eqs. (14) through (16)]. To complete the definition of the problem, we will assume as given a set of initial and boundary conditions that make the problem well posed. Imposed currents and charges can also be specified as independent sources; for example, a term such as $\mathbf{J}_{v}=\rho \mathbf{v}$ is very common in problems deriving from particle accelerator design.

\section{Domain Discretization}

The space-time domain is discretized by the reference strategy by using two dual oriented cell complexes, which act as primary and secondary meshes.* We assume that each mesh is obtained as a Cartesian product of the elements of a cell complex, which subdivides the problem domain in space, by those of a cell complex discretizing the time interval for which a solution is sought. The more complex case of moving meshes, and the even more general case of generic space-time cell complexes, could be contemplated as well but entail a number of difficulties in the attribution of a physical meaning to the quantities and the deduction of suitable constitutive equations (Nguyen, 1992), which we choose to avoid in this context. Remember, however, that the reference method can be extended to include these cases as well.

Given this choice, we have for $p=0,1,2,3$ four collections of indexed primary $p$-cells $\left\{\tau_{p}^{i}, i=1, \ldots, n_{p}\right\}$ in space. To each primary $p$-cell $\tau_{p}^{i}$ there corresponds a dual secondary $(3-p)$-cell $\tilde{\tau}_{3-p}^{i}$ with the same index and the default orientation defined by that of the dual primary cell. The secondary cells also constitute, therefore, four collections of $p$-cells $\left\{\tilde{\tau}_{p}^{i}, i=1, \ldots, \tilde{n}_{p}=n_{3-p}\right\}$ (Fig. 33). Notice that as a way to facilitate drawing, the 1- and 2-cells in Figure 33 and in Figures 35 and 36, are straight or planar, but this is not required by the definition of cell, on which the reference method is based. However, the

\footnotetext{
*In fact, the reference discretization strategy as described next applies to nondual primary and secondary meshes as well, and in particular to the case of geometrically coincident (although logically distinct) primary and secondary meshes (this last choice simplifies the setup of boundary conditions and the treatment of material discontinuities). However, if we would use nondual meshes, some significant algebraic properties of the discretized model based on dual meshes (in particular, operators' adjointness) would be at risk of being lost.
} 


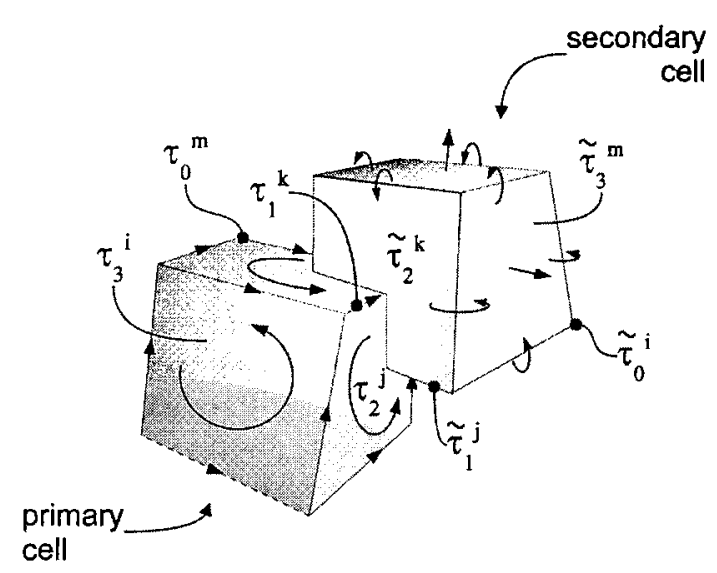

FIGURE 33. Reference discretization of the domain in space. Note that the orientation and index of each primary $p$-cell are used to index and orient the dual secondary $(3-p)$-cell (the orientation of 0 -cells and 3-cells is not represented).

use of planar and straight cells greatly simplifies the calculations, especially for what concerns the discretization of the constitutive relations.

The actual construction of the two meshes is problem dependent, since it must consider what kind of boundary conditions are specified (which determines what kind of cells are needed at the boundary); where the material discontinuities, if any, are located; and to what constitutive parameter they refer. This last point will become clearer after the discussion on constitutive relations discretization (in Section IV.A.3). In general, one can start by defining a primary mesh that conforms to material and domain boundaries, and then construct the secondary cells by defining within each $n$-cell of the primary mesh ( $n$ is the dimension of the domain) a secondary 0 -cell. The secondary mesh is then built by starting from this 0 -cell so as to make the two cell complexes reciprocally duals. The actual position of each secondary 0 -cell within its dual $n$-cell also depends on the problem and on the strategy adopted for the discretization of constitutive relations. In many cases, the position corresponding to the barycenter of the $n$-cell is a good choice, but, as will be hinted at subsequently, it is not always the optimal one.

The domain in time is a time interval $I$ subdivided by two dual cell complexes. The primary one is constituted by two collections of indexed $p$-cells, with $p=0,1$. The 0 -cells are time instants $\left\{t_{p}^{n}, n=1, \ldots, N\right\}$ indexed according to increasing time. As a way to simplify the notation and facilitate the comparison with existing methods, the time interval going from $t_{0}^{n}$ to $t_{0}^{n+1}$ is indexed as $t_{1}^{n+1 / 2}$. In time, to each primary cell $t_{p}^{n}$ there corresponds a dual secondary cell $\tilde{t}_{1-p}^{n}$, inheriting the index of its dual cell. Thus, the time interval $\tilde{t}_{1}^{n}$ goes from the time instant $\tilde{t}_{0}^{n-1 / 2}$ to the time instant $\tilde{t}_{0}^{n+1 / 2}$ (Fig. 34). Primary 


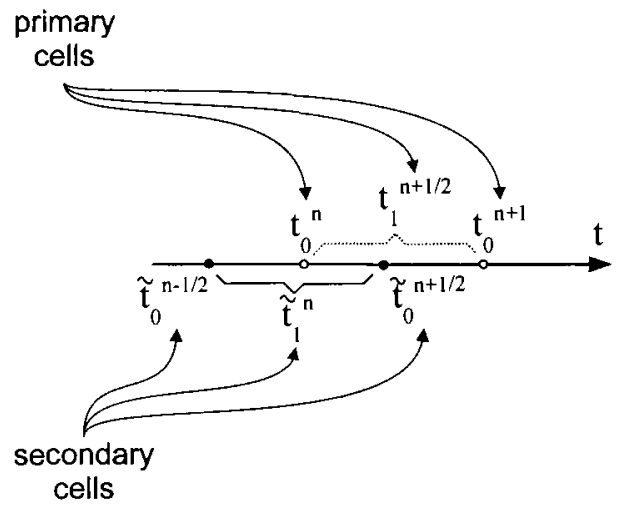

FIGURE 34. Reference discretization of the domain in time.

space-time cells are obtained as Cartesian products $\tau_{p}^{i} \times t_{q}^{n}$, and secondary ones as products $\tilde{\tau}_{p}^{i} \times \tilde{t}_{q}^{n}$. Note that the duality of the meshes applies in both space and time.

This discretization supplies the oriented geometric objects needed to support the global physical quantities of electromagnetism; that is, $Q^{\rho}, Q^{j}, \phi^{b}$, $\phi^{e}, \psi^{d}, \psi^{h}, \mathcal{U}^{a}$, and $\mathcal{U}^{v}$ [defined in Eqs. (48) through (55)]. However, the quantities actually appearing in the formulas of the reference strategy are $Q^{j}, \phi^{b}, \phi^{e}, \psi^{d}$, and $\psi^{h}$ only. The association of a global quantity with a geometric object will be denoted by a pair of indexes, according to the following convention:

$$
\begin{aligned}
\phi^{b}\left(\tau_{2}^{i} \times t_{0}^{n}\right) & =\phi_{i, n}^{b} \\
\phi^{e}\left(\tau_{1}^{i} \times t_{1}^{n+1 / 2}\right) & =\phi_{i, n+1 / 2}^{e} \\
\psi^{d}\left(\tilde{\tau}_{2}^{i} \times \tilde{t}_{0}^{n+1 / 2}\right) & =\psi_{i, n+1 / 2}^{d} \\
\psi^{h}\left(\tilde{\tau}_{1}^{i} \times \tilde{t}_{1}^{n}\right) & =\psi_{i, n}^{h} \\
Q^{j}\left(\tilde{\tau}_{2}^{i} \times \tilde{t}_{1}^{n}\right) & =Q_{i, n}^{j}
\end{aligned}
$$

\section{Topological Time Stepping}

Previously, we explained how Faraday's law and Maxwell-Ampère's law can be given a geometric interpretation in terms of global quantities associated with a space-time cylinder (Figs. 12 and 13). Within a domain discretized following the prescriptions of the previous subsection, we can apply this property to build a topological time-stepping procedure. Faraday's law is used to time-step $\phi^{b}$ as follows. We build a space-time cylinder on a primary 2-cell $\tau_{2}^{i}$ 


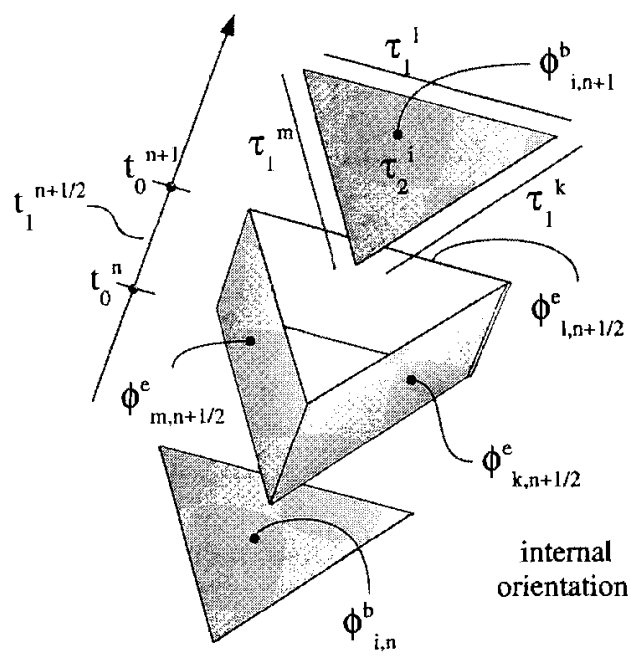

FIGURE 35. Blown-up representation of the geometric objects and global physical quantities involved in topological time stepping on the primary mesh. The (internal) orientation of the geometric objects is not represented.

considered at the time instant $t_{0}^{n}$. The resulting 2-cell $\tau_{2}^{i} \times t_{0}^{n}$ is the first base of the cylinder. The boundary $\partial \tau_{2}^{i}$ of $\tau_{2}^{i}$, considered during the time interval $t_{1}^{n+1 / 2}$, is a finite collection of 2-cells $\partial \tau_{2}^{i} \times t_{1}^{n+1 / 2}=\sum_{k}\left[\tau_{2}^{i}, \tau_{1}^{k}\right] \tau_{1}^{k} \times t_{1}^{n+1 / 2}$ that constitutes the lateral surface of the cylinder. The cylinder is closed by the 2-cell $\tau_{2}^{i} \times t_{0}^{n+1}$ (Fig. 35). If we assume as known the primary global quantities at times $t<t_{0}^{n+1}$, that is, $\phi_{i, n}^{b}$ and $\phi_{k, n+1 / 2}^{e}$, we can calculate exactly $\phi_{i, n+1}^{b}$ from the topological equation $\delta \Phi^{2}=0^{3}$ [Eq. (131)], which, if we isolate the unknown term, becomes

$$
\phi_{i, n+1}^{b}=\phi_{i, n}^{b} \pm \sum_{k=1}^{n_{1}}\left[\tau_{2}^{i}, \tau_{1}^{k}\right] \phi_{k, n+1 / 2}^{e}
$$

where the actual sign of the second term of the right side depends on the default orientation assumed for the primal 2-cells to which a positive $\phi^{e}$ is associated. Using the representation [Eq. (106)] of cochains as vectors of global physical quantities and the definition [Eq. (88)] of the incidence matrices, we can rewrite the topological time-stepping formula [Eq. (188)] in matrix terms, as follows:

$$
\Phi_{n+1}^{b}=\Phi_{n}^{b}-\mathbf{D}_{2,1} \Phi_{n+1 / 2}^{e}
$$

where we assume the default orientation which gives to the last term a minus sign. 


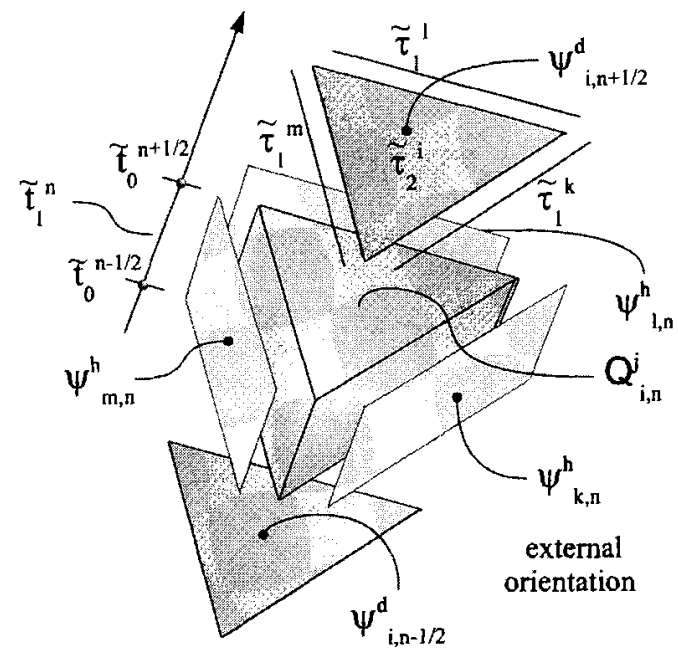

Flgure 36. Blown-up representation of the geometric objects and global physical quantities involved in topological time stepping on the secondary mesh. The (external) orientation of the geometric objects is not represented.

An analogous procedure holds for the time stepping of $\psi^{d}$ by means of Maxwell-Ampère's law $\delta \tilde{\Psi}^{2}=\tilde{\mathbf{Q}}^{3}$ on the secondary mesh (Fig. 36). The result is

$$
\psi_{i, n+1 / 2}^{d}=\psi_{i, n-1 / 2}^{d} \pm \sum_{k}\left[\tilde{\tau}_{2}^{i}, \tilde{\tau}_{1}^{k}\right] \psi_{k, n}^{h} \pm Q_{i, n}^{j}
$$

where $Q_{i, n}^{j}$ is the charge associated by the electric current flowing through $\tilde{\tau}_{2}^{i}$ during the time interval $\tilde{t}_{1}^{n}$. With a suitable choice of default orientations, the matricial representation of Eq. (190) corresponding to Eq. (189) is

$$
\tilde{\Psi}_{n+1 / 2}^{d}=\tilde{\Psi}_{n-1 / 2}^{d}+\tilde{\mathbf{D}}_{2,1} \tilde{\Psi}_{n}^{h}-\tilde{\mathbf{Q}}_{n}^{j}
$$

If we consider the collection $\left\{\phi_{i, 0}^{b}\right\}$ given as part of the initial conditions, we can use Eq. (188) to start a time stepping for $\phi^{b}$, provided the set of values $\left\{\phi_{k, 1 / 2}^{e}\right\}$ is known. Of course, we cannot expect these values to be also given as initial conditions. We can, however, assume the set of values $\left\{\psi_{i, 1 / 2}^{d}\right\}$ to be given as initial conditions. Hence, we can derive $\left\{\phi_{k, 1 / 2}^{e}\right\}$ from $\left\{\psi_{i, 1 / 2}^{d}\right\}$ by means of a discrete constitutive link $F_{\varepsilon^{-1}}$, and advance in time in this way $\phi^{b}$ from $\left\{\phi_{i, 0}^{b}\right\}$ to $\left\{\phi_{i, 1}^{b}\right\}$. At this point we know $\left\{\phi_{i, 1}^{b}\right\}$ and we can derive $\left\{\psi_{i, 1}^{h}\right\}$ from it by means of a discrete constitutive link $F_{\mu^{-1}}$, and $\left\{Q_{i, 1}^{j}\right\}$ from $\left\{\phi_{j .1 / 2}^{e}\right\}$ and $\left\{\phi_{j, 3 / 2}^{e}\right\}$ or, better, indirectly from $\left\{\psi_{i, 1 / 2}^{d}\right\}$ and $\left\{\psi_{i, 3 / 2}^{d}\right\}$ by means of a constitutive link 


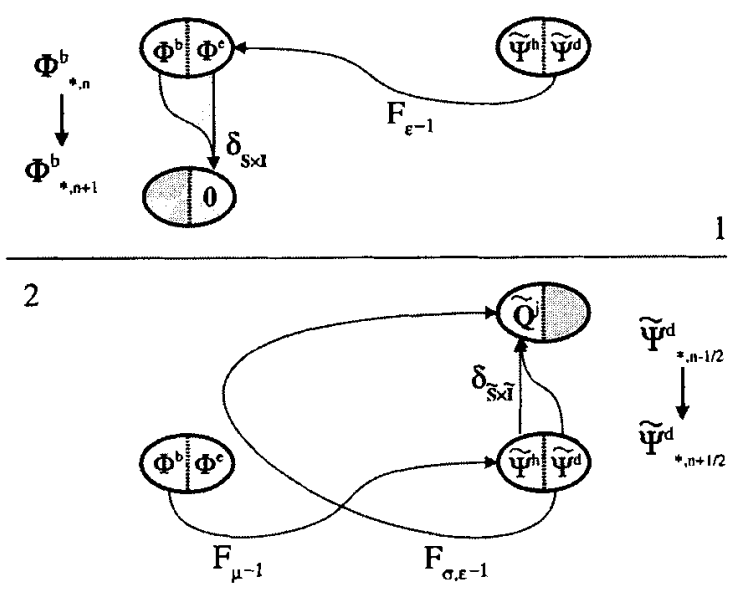

FIGURE 37. The two half-time steps of the reference method. Topological time stepping is applied on each side of the diagram, to update $\phi^{b}$ and $\psi^{d}$, respectively. The discrete constitutive links supply the quantities required by the time-stepping formulas; that is, $\phi^{e}$ for the updating of $\phi^{b}$, and $\psi^{h}$ and $Q^{j}$ for the updating of $\psi^{d}$.

$F_{\sigma, \varepsilon^{-1}}$ which includes the action of the constitutive link $f_{\varepsilon^{-1}}$ and of $f_{\sigma}$. This allows the determination of $\left\{\psi_{i, 3 / 2}^{d}\right\}$ by time-stepping $\left\{\psi_{i, 1 / 2}^{d}\right\}$ using Eq. (190), and so on (Fig. 37). In matricial representation, for a generic time step $n$, this corresponds to

$$
\begin{aligned}
\Phi_{n+1}^{b} & =\Phi_{n}^{b}-\mathbf{D}_{2,1} F_{\varepsilon^{-1}}\left(\tilde{\Psi}_{n+1 / 2}^{d}\right) \\
\tilde{\Psi}_{n+1 / 2}^{d} & =\tilde{\Psi}_{n-1 / 2}^{d}+\tilde{\mathbf{D}}_{2,1} F_{\mu^{-1}}\left(\Phi_{n}^{b}\right)-F_{\sigma, \varepsilon^{-1}}\left(\tilde{\Psi}^{d}\right)
\end{aligned}
$$

where in the term $F_{\sigma, \varepsilon^{-1}}\left(\tilde{\Psi}^{d}\right)$ the cochain has no time-step subscript, as a reminder that both $\tilde{\Psi}_{n+1 / 2}^{d, c}$ and $\tilde{\Psi}_{n-1 / 2}^{d}$ are involved in the link.

The topological time-stepping formulas [Eqs. (188) and (190)] are based on two of Maxwell's four equations. We will now prove that in adopting the time-stepping scheme thus described, we will not need to explicitly enforce the other pair of Maxwell's equations. As usual for numerical methods devoted to time-domain electromagnetic problems, it will suffice to show that, if these equations are satisfied at a given time instant, they remain so after the execution of a time step. Consider first Gauss's magnetic law. This asserts the vanishing of magnetic flux associated with the boundary of any 3-cell $\tau_{3}$ at any time instant $t_{0}^{n}$. To simplify the notation, we denote with $\Phi_{n}$ the magnetic flux cochain at time $t_{0}^{n}$, thus avoiding the use of products of spacelike and timelike geometric objects in the formulas. With this provision, Gauss's law for a particular 3-cell 
$\tau_{3}^{*}$, considered at time $t_{0}^{n}$, reads as follows:

$$
\phi^{b}\left(\partial \tau_{3}^{*} \times t_{0}^{n}\right)=\left(\partial \tau_{3}, \Phi_{n}\right)=0
$$

where, in the middle term, we have represented the quantity in terms of a chain-cochain pairing. We must now show that, from the validity of Eq. (194) and the application of the time-stepping formula [Eq. (188)], there follows the validity of Gauss's law at time $t_{0}^{n+1}$. Substituting in Eq. (194) the expression [Eq. (96)] of the boundary in terms of incidence numbers, we have

$$
\phi^{b}\left(\partial \tau_{3}^{*} \times t_{0}^{n}\right)=\left(\sum_{i}\left[\tau_{3}^{*}, \tau_{2}^{i}\right] \tau_{2}^{i}, \Phi_{n}\right)=\sum_{i}\left[\tau_{3}^{*}, \tau_{2}^{i}\right] \phi_{i, n}^{b}=0
$$

The same substitution, applied to the expression of $\phi^{b}$ at time $t_{0}^{n+1}$, gives

$$
\phi^{b}\left(\partial \tau_{3}^{*} \times t_{0}^{n+1}\right)=\sum_{i}\left[\tau_{3}^{*}, \tau_{2}^{i}\right] \phi_{i, n+1}^{b}
$$

Substituting the time-stepping formula of Eq. (188) in the right side of Eq. (196), we obtain

$$
\sum_{i}\left[\tau_{3}^{*}, \tau_{2}^{i}\right] \phi_{i, n+1}^{b}=\sum_{i}\left[\tau_{3}^{*}, \tau_{2}^{i}\right] \phi_{i, n}^{b} \pm \sum_{i}\left[\tau_{3}^{*}, \tau_{2}^{i}\right] \sum_{k}\left[\tau_{2}^{i}, \tau_{1}^{k}\right] \phi_{k . n+1 / 2}^{e}
$$

Rearranging the terms, we have

$$
\sum_{i}\left[\tau_{3}^{*}, \tau_{2}^{i}\right] \phi_{i, n+1}^{b}=\sum_{i}\left[\tau_{3}^{*}, \tau_{2}^{i}\right] \phi_{i, n}^{b} \pm \sum_{i} \sum_{k}\left[\tau_{3}^{*}, \tau_{2}^{i}\right]\left[\tau_{2}^{i}, \tau_{1}^{k}\right] \phi_{k, n+1 / 2}^{e}
$$

The first term on the right side of Eq. (198) vanishes, since we have assumed Eq. (195) to hold true; the second term vanishes in virtue of the relation [Eq. (90)] holding among incidence numbers. Hence, remembering Eq. (196), we finally have

$$
\phi^{b}\left(\partial \tau_{3}^{*} \times t_{0}^{n+1}\right)=0
$$

This proves that if Gauss's magnetic law is satisfied at time $t_{0}^{n}$, then, upon application of the topological time-stepping formula, it is also satisfied at time $t_{0}^{n+1}$. From Eq. (198) there follows a more general conclusion, namely, that following the execution of topological time stepping, the amount of violation of Gauss's magnetic law, if any, does not change. In other words, the topological time stepping on $\phi^{b}$ automatically enforces the law of magnetic charge conservation.

In the case of Gauss's electric law the balance to be enforced is that between the electric charge associated with the 3-cells and the electric flux through its 
boundary. Suppose that at time $\tilde{t}_{0}^{n-1 / 2}$ there is a charge $Q_{*, n-1 / 2}^{\rho}$ associated with the 3-cell $\tilde{\tau}_{3}^{*}$, and that the following relation holds:

$$
\psi^{d}\left(\partial \tilde{\tau}_{3}^{*} \times \tilde{t}_{0}^{n-1 / 2}\right)=Q_{*, n-1 / 2}^{\rho}
$$

Repeating the steps of the preceding proof, but using instead the time-stepping formula of Eq. (190), we obtain

$$
\psi^{d}\left(\partial \tilde{\tau}_{3}^{*} \times \tilde{t}_{0}^{n+1 / 2}\right)=Q_{*, n-1 / 2}^{\rho} \pm \sum_{i}\left[\tilde{\tau}_{3}^{*}, \tilde{\tau}_{2}^{i}\right] Q_{i, n}^{j}
$$

This shows that after topological time stepping, the electric flux associated with the boundary of $\tilde{\tau}_{3}^{*}$ may have changed. However, this change is consistent with the law of charge conservation, since the new term on the right side of Eq. (201) is the result of the electric current flowing through the boundary of the cell during the time interval $\tilde{t}_{1}^{n}$. Hence, the topological time stepping on $\psi^{d}$ enforces automatically the law of electric charge conservation and preserves the violation of Gauss's electric law, if any.

Note how the realization of the space-time nature of topological laws suggests the adoption of a uniquely determined time-stepping procedure on each side of the factorization diagram of physical quantities, an observation that is true for any theory admitting such a factorization diagram. It is clear that we are revealing here the roots of the adoption, within many numerical methods for partial differential equations, of a leapfrog time-stepping procedure based on two half-time steps. This choice, in the absence of the justification given in the present work, which is based on the analysis of the structure of physical theories, is often considered as an oddity, justified only by the good results it offers. [For further details on the topological time-stepping process see the discussion in Mattiussi (2001).]

The limits that the univocity of the topological time-stepping process puts on the form of the complete time stepping are not as severe as might appear at first. It is indeed true that the topological time-stepping formulas (189) and (191) are based on a topological law applied to a single, space-time 3-cell, and, therefore, that each newly calculated value directly depends only on quantities associated with the cell itself and with its boundary. The complete time-stepping operator includes, however, in addition to the topological relation, at least one discrete constitutive operator. This constitutive operator links the quantities directly involved in the topological time-stepping formula to other quantities. The constitutive link, therefore, allows the extension both in space and in time of the dependence of the newly calculated value on quantities associated with cells other than those for which the topological law is enforced. Thus, the newly calculated values associated with a given cell can be made to depend on quantities associated with cells of a generic neighborhood of that cell in space, and extending in time deeper into the past than the single time step considered 
by the topological relation, or on other quantities for the time instant at which the new quantity is calculated. This can be expressed by rewriting the particular time-stepping formulas of Eqs. (192) and (193) as follows:

$$
\begin{aligned}
\Phi_{n+1}^{b} & =\Phi_{n}^{b}-\mathbf{D}_{2,1} F_{\varepsilon^{-1}}\left(\tilde{\Psi}^{d}\right) \\
\tilde{\Psi}_{n+1 / 2}^{d} & =\tilde{\Psi}_{n-1 / 2}^{d}+\tilde{\mathbf{D}}_{2,1} F_{\mu^{-1}}\left(\Phi^{b}\right)-F_{\sigma, \varepsilon^{-1}}\left(\tilde{\Psi}^{d}\right)
\end{aligned}
$$

that is, removing the time subscript from the cochains entering the discrete constitutive links in order to emphasize the involvement of the whole space-time cochain in the link. Observe that if the expression of the discrete constitutive operators is explicitly given, by actually substituting them in the topological time-stepping formulas, we can make them depend on only two kinds of variables, in this case $\phi^{b}$ and $\psi^{d}$ (but other pairs of variables can be selected to appear in the formulas, applying differently the constitutive links).

In summary, there is a possibility of building a variety of time-stepping procedures (including implicit ones) complying with the adoption of a topological time-stepping operator. Given the variety of discrete constitutive links that can be built, and the uniqueness of the topological time-stepping links, one could, therefore, conceive a numerical package offering the choice between different discretization strategies for constitutive relations, to be combined with the unique discretization of topological laws based on the coboundary operator. Note finally that we can expect problems in trying to use a weighted residual approach to build a topological time-stepping procedure, for the geometric ideas upon which we have based the topological time-stepping procedure cannot be easily extended to spread cells.

\section{Strategies for Constitutive Relations Discretization}

The task of constitutive relations discretization consists of determining a link between cochains which approximates the local constitutive equation and, with it, the ideal material behavior it represents. There are many possible approaches to this task, and from this point of view the three cases presented next are in no way exhaustive. However, since many numerical methods do not consider explicitly the particular problem represented by the discretization of constitutive relations and perform this task in a manner that appears at most as some form of educated guessing, we will try at least to present discretization procedures for constitutive relations that can be applied systematically. Our inspiration, as usual, comes from existing numerical methods. We will first consider one of the simplest and most intuitive approaches that qualify as a systematic technique for the discretization of constitutive relations; then two more sophisticated classes of strategies are presented. 
Remark IV.I The discretization of constitutive relations is often referred to as the discretization of the Hodge star operator * (Tarhasaari et al., 1999; Teixeira and Chew, 1999b; see Bamberg and Sternberg, 1988, for a formal definition of its action). Considering the great variety of possible constitutive links, this point of view appears too restrictive. The Hodge star operator institutes indeed a one-to-one correspondence between ordinary $p$-forms $\alpha^{p}$ and twisted $(n-p)$-forms $\tilde{\beta}^{n-p}$ defined on an $n$-dimensional manifold. We can represent this relation as follows:

$$
\tilde{\beta}^{n-p}=* \alpha^{p}
$$

It is apparent from Eq. (204) that if we adopt the representation of fields as differential forms, the Hodge operator can play the part of a constitutive link (provided we include within it the required material parameters). However, in this role, the Hodge operator is a mathematical model for the behavior of a particular class of ideal materials (and when it is considered merely in mathematical terms, that is, without the intervention of material parameters, not even that), and cannot be considered as a model for all material behaviors. It is the fact that the Hodge star operator constitutes the traditional bridge between ordinary and twisted forms that tempts us to consider it the constitutive operator. That things are not so can be seen by considering that constitutive equations can be mathematically much more complex than the simple correspondence brought about by the Hodge operator (see, for example, the discussion in Post, 1997). Of course, since the transition from ordinary to twisted differential forms (or vice versa) is implied by the constitutive links, the Hodge operator or something analogous, capable of "crossing the bridge" in the factorization diagram, will be required, but typically only as a part of the complete constitutive link. In other words, every operator linking two differential forms that represent two fields plays the role of the constitutive relation of a particular ideal material [perhaps a nonphysical one, as in the case of materials which form the so-called perfectly matched layer, used for the implementation of absorbing boundary conditions (Berenger, 1994; Teixeira and Chew, 1999a)]. However, contrary to what happens with the topological equations, no single operator can claim a privileged role as constitutive operator.

\section{a. Discretization Strategy 1: Global Application of Local}

\section{Constitutive Statements}

While introducing the idea of constitutive links, we hinted at the fact that a local constitutive equation of the kind $\mathbf{D}=\varepsilon \mathbf{E}$ holds true also in a macroscopic space-time region, provided that the fields are uniform in space and constant in time, and that the material is homogeneous. In this case, if the surface $S$ to which the electric flux is associated is planar, and orthogonal to the straight 
line segment $L$ to which the voltage is associated, we can write

$$
\frac{\psi^{d}}{S}=\varepsilon \frac{\phi^{e}}{L I}
$$

where $I$ is the time interval during which the voltage is considered and, with some notational abuse, we have identified the symbols of the geometric objects with the value of their extension. The uniformity conditions upon which the transition from the local statement to the global one is based are admittedly severe. Consequently these requirements are not satisfied in the majority of cases, and equations such as Eq. (205) are, therefore, only rough approximations of the actual relation holding between the global variables. Nonetheless, in many numerical methods this approach is adopted more or less explicitly in order to obtain a discrete version of the constitutive links.

The rationale behind this choice is that when we decrease the maximum space and time discretization steps, the uniformity hypothesis is approached more and more closely and Eq. (205) becomes an acceptable discrete approximation of $\mathbf{D}=\varepsilon \mathbf{E}$. Note that in addition to uniformity there is a requirement of geometric regularity and orthogonality of the geometric objects and, therefore, of the discretization meshes. Hence, this approach will require meshes with dual, orthogonal cells, for example, the regular orthogonal grids of the FDTD method or the more general Delaunay-Voronoi meshes (Guibas and Stolfi, 1985). This last requirement, however, can usually be relaxed, paying the price for a more complex evaluation of the terms appearing in the link, to take care, for example, of the angles between the cells or their curvature.

In summary, applying consistently this simple discretization technique to the local expression of all the constitutive relations appearing in a problem, one obtains a series of links between cochains, which are the required discrete constitutive links. Since this approach can give only very crude approximations of the actual constitutive relations, it is acceptable only if the fields do not vary rapidly in space and time or if the recourse to a very fine mesh can be accepted. To find, for the constitutive relations, a more accurate discrete approximation than the one just presented, we must use more extensively the information represented by the local constitutive equations. We will consider, in the next subsections, two approaches based on the preliminary reconstruction-based on the corresponding cochains - of one or both of the field functions appearing in the constitutive equation written in local form.

\section{b. Discretization Strategy 2: Field Function Reconstruction and Projection}

The method considered in the present section requires the reconstruction of only one of the field functions appearing in the constitutive equation in local form. An example will clarify the actual workings of the strategy. Suppose that 
we want to discretize the following constitutive equation:

$$
\mathbf{B}=f_{\mu}(\mathbf{H})
$$

As usual, to simplify the notation we represent the field functions using the usual tools of vector calculus, even though a formulation in terms of differential forms would be more appropriate (see Teixeira and Chew, 1999b, for a description of the strategy both in differential forms and in vector calculus language). In the discrete setting of the reference strategy, the field functions $\mathbf{B}$ and $\mathbf{H}$ appearing in Eq. (206) do not belong to the problem's variables, and we have instead the magnetic flux cochain $\Phi^{b}$ and the electric flux cochain $\tilde{\Psi}^{h}$, which at the end of the process must be linked by the relation $\Phi^{b}=F_{\mu}\left(\tilde{\Psi}^{h}\right)$. In order to use the information constituted by Eq. (206), we proceed by deriving from the cochain $\tilde{\Psi}^{h}$ a field function $\mathbf{H}$. To this end, we select a reconstruction operator $\mathbf{R}_{h}$ giving for each cochain $\tilde{\Psi}^{h}$ a field function $\mathbf{H}$, as follows:

$$
\mathbf{H}=\mathrm{R}_{h}\left(\tilde{\Psi}^{h}\right)
$$

Note that the reconstruction in Eq. (207) starts from space-time global quantities, and, therefore, the reconstructed field is intended as given in spacetime also, as a function $\mathbf{H}(\mathbf{r}, t)$. We can now apply to $\mathbf{H}$ the local constitutive link [Eq. (206)], obtaining the field function $\mathbf{B}$. We must finally return to cochains, and we do this by means of a projection operator $P^{b}$, which produces a cochain $\Phi^{b}$ for each field function $\mathbf{B}$, as follows:

$$
\Phi^{b}=\mathrm{P}^{b}(\mathbf{B})
$$

The composition of the operators [Eqs. (207), (206), and (208)], gives the desired discrete constitutive link $F_{\mu}$ :

$$
\Phi^{b}=F_{\mu}\left(\Psi^{h}\right)=\mathrm{P}^{b}\left(f_{\mu}\left(\mathrm{R}_{h}\left(\tilde{\Psi}^{h}\right)\right)\right)
$$

The same approach applies to the discretization of the electrostatic link, and of any other constitutive relation, of which the local expression corresponding to Eq. (206) is known (Fig. 38). A natural joint requirement for reconstruction and projection operators is that for every cochain $\mathbf{c}^{p}$ the following relation holds true:

$$
\mathrm{P}^{*}\left(\mathrm{R}_{*}\left(\mathrm{c}^{p}\right)\right)=\mathbf{c}^{p}
$$

That is, by projecting back the reconstructed field one must obtain the original cochain. This means that the combined operator $P^{*} R_{*}$ must be the identity operator. Note, however, that this is not true in general for the operator $R_{*} P^{*}$; that is, because of the limitations of the reconstruction operator, by projecting a generic field and then reconstructing it one typically does not obtain the original field (Tarhasaari et al., 1999). 


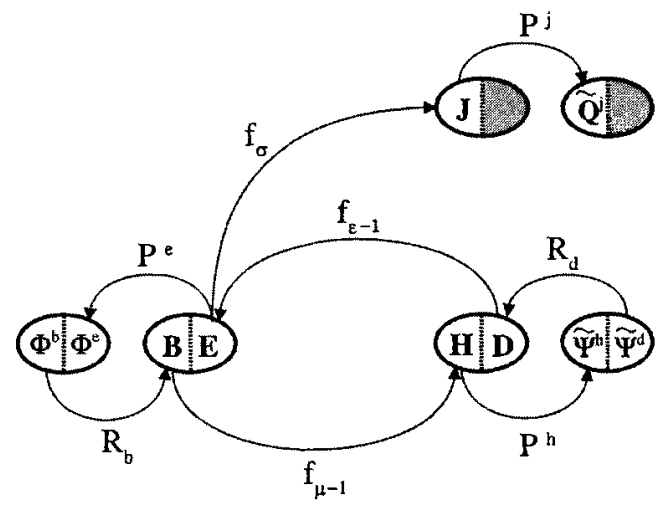

FIgURE 38. The reconstruction-projection method for the discretization of constitutive relations. Given the starting cochain, an approximation of the corresponding field function is determined by means of a reconstruction operator $R_{*}$. The result is then subjected to the action of one or more local constitutive operators $f_{*}$. The resulting field function is finally projected on the cell complex by means of an operator $\mathrm{P}^{*}$; thus one recovers a cochain.

Note also that in Eqs. (207) and (208) we added to the symbols $R$ and $P$ of the reconstruction and projection operators an index, which refers to the field function involved in the process. This was done to serve as a reminder that the operators must comply with the association of physical quantities with oriented geometric objects, so that each operator will be "tailored" to the actual nature of the fields it is called to operate on. In particular, the reconstruction operator operates on $p$-cochains and produces $p$-fields that must be compatible with the original cochain. It is clear, therefore, that the proper selection of the reconstruction operator is instrumental in the attainment of a good discrete solution. For this reason, subsequently a separate section is devoted to the discussion of some actual reconstruction operators. Using the just-derived representation of the discrete constitutive operators, we can rewrite the generic time-stepping formulas (202) and (203) for this particular discretization strategy, as follows:

$$
\begin{aligned}
\Phi_{n+1}^{b} & =\Phi_{n}^{b}+\mathbf{D}_{2,1} \mathrm{P}^{e}\left(f_{\varepsilon^{-1}}\left(\mathrm{R}_{d}\left(\tilde{\Psi}^{d}\right)\right)\right) \\
\tilde{\Psi}_{n+1 / 2}^{d} & =\tilde{\Psi}_{n-1 / 2}^{d}+\tilde{\mathbf{D}}_{2,1} \mathrm{P}^{h}\left(f_{\mu^{-1}}\left(\mathrm{R}_{b}\left(\Phi^{b}\right)\right)\right)+\mathrm{P}^{j}\left(f_{\sigma}\left(f_{\varepsilon^{-1}}\left(\mathrm{R}_{d}\left(\tilde{\Psi}^{d}\right)\right)\right)\right)
\end{aligned}
$$

With the reconstruction and projection operators appearing in Eqs. (211) and (212), the reference discretization strategy becomes a class of numerical methods complying with the reference discretization strategy. Let us analyze in general terms where the discretization error might enter this class of methods. The strategy first requires reconstructing the field function by starting from a cochain. This opens the first door to possible errors since we cannot expect the 
true solution to be in the range of our reconstruction operator. Remembering what has been said about limit systems and the concept of field as a collection of its manifestations in terms of cochains on the directed set of all the cell complexes that subdivide the domain, we can interpret this by saying that a single cochain alone cannot determine the field it derives from. More precisely, given a cell complex and a cochain defined on it, there would be in general an infinite number of fields that admit that cochain as its projection on that complex. The choice of the reconstruction operator corresponds, therefore, to the selection of a particular field in the multiplicity of fields that are compatible with the discrete image we start from.

After the reconstruction step and the application of the local constitutive operator, we project the resulting field function onto the cell complex, obtaining a cochain, and we impose on it the topological equation. Since the cell complex is finite, it follows that we are enforcing only a finite subset of all the possible topological relations implied by the corresponding topological laws. This gives more freedom to the solution than what was implied by the original physical field problem. It is the combination of these two processes that gives rise to the discretization error that we will finally find in the solution of the discrete problem. This double nature of the discretization error was lucidly analyzed by Schroeder and Wolff (1994). The reconstruction-projection strategy for the discretization of constitutive relations, and the corresponding error analysis, was given a formal treatment based on the concepts of the theory of categories (Tarhasaari et al., 1999). In this context, the names Whitney functor and de Rham functor were suggested for the reconstruction and projection operators, respectively.

Let us further comment regarding the properties of the projection operator. Speaking of limit systems, we said that a field can be thought of as a collection of cochains, each of which is a projection of the field on a particular cell complex. Adopting the natural representation of a cochain as a vector of global physical quantities associated with cells, the operation of projection amounts, therefore, to the evaluation of these global quantities on the $p$-cells of the complex, where $p$ and the complex orientation must suit the nature of the field. In practice, this evaluation corresponds usually to the integration of the field function on these $p$-cells. This fact, combined with the fact that the reconstruction operator performs an approximation of the field function, opens the door to some optimization opportunities. We know from the theory of approximation that the reconstructed function typically has a set of loci where the approximation is of a higher order than that at generic points of the domain. Therefore, by making the cells where the projection is performed coincide with these loci, we can obtain a higher accuracy in the resulting discrete constitutive equation. This means that the accuracy of the results can benefit from the proper selection and placement of the primary and secondary meshes. In particular, it can be shown that the choice of two suitably placed dual grids can 
be desirable also in this respect (Mattiussi, 1997). For low-order polynomial reconstructions on regular cells, the center of the cells is usually the optimal location for the dual object. This extends to the time-stepping procedure, where it suggests the placement of secondary time instants in the middle of primary time intervals. However, for higher-order polynomial and for nonpolynomial reconstruction, as well as for nonregular cells, the determination of the optimal reciprocal position and shape of the cells of the two cell complexes can be far from obvious. The solution of this problem requires usually the solution of an algebraic or a differential system of equations which, in turn, are derived by enforcing some best approximation constraints. [See Mattiussi (1997) for a description of some possible approaches to this problem.]

Note that the approach used in the reconstruction-projection strategy to discretize the constitutive relations gives rise to discrete links where the value of the resulting cochain on each cell depends on the values taken by the cochain one starts with on many cells, potentially on all those entering the reconstruction process. So that the sparsity of the matrices appearing in the system of algebraic equations is preserved, the reconstruction process is usually performed locally, so that the value of the reconstructed field function on each point depends only on the values taken by the original cochain on the cells of a sufficiently small neighborhood of the point. In particular, the simple discretization strategy described in the previous subsection is a particular case of the strategy described here. In that case the reconstruction operator works locally on a single cell, giving a uniform field, which is projected onto the dual cell.

\section{c. Discretization Strategy 3: Error-Based Discretization}

There is another approach to the discretization of constitutive relations which is based on the reconstruction of field functions. Let us describe the workings of this strategy by using the same example of the previous strategy. As before, we assume as known the electric flux cochain $\tilde{\Psi}^{h}$ and we want to determine the magnetic flux cochain $\Phi^{b}$. Contrary to the previous case, however, we apply a reconstruction operator to both cochains as follows:

$$
\begin{aligned}
& \mathbf{H}=\mathrm{R}_{h}\left(\tilde{\Psi}^{h}\right) \\
& \mathbf{B}=\mathrm{R}_{b}\left(\Phi^{b}\right)
\end{aligned}
$$

Since the cochain $\Phi^{b}$ is the unknown term of the discrete link, the reconstruction of $\mathbf{B}$ is made in formal terms only. Ideally, the relation holding between $\mathbf{B}$ and $\mathbf{H}$ is the local constitutive equation $\mathbf{B}=f_{\mu}(\mathbf{H})$. From the discussion of the previous subsection we know that, both fields being obtained by reconstruction from cochains, these fields are forced to be in the range of the reconstruction operators. Consequently, we cannot expect the local constitutive equation to be satisfied exactly by the reconstructed fields. 
We, therefore, define an error density function in the domain, which we denote with

$$
\xi(\mathbf{B}, \mathbf{H})
$$

which is intended to give a local estimate of the amount of the violation of the constitutive link. We ask, as the minimal set of requirements for this scalar function which measures the local constitutive error, for it to always be positive and to vanish only for $\mathbf{B}$ and $\mathbf{H}$ satisfying $\mathbf{B}=f_{\mu}(\mathbf{H})$. The actual definition of the function $\xi$ is a nontrivial task, which depends on the problem and on its constitutive relations. We will not consider in detail this subject here, assuming this function as given. For this important topic the reader is referred to the literature, in particular to that dealing with complementary variational techniques, which appear especially suited to the determination of physically significant local error functions linked to local and global energy estimates (Albanese and Rubinacci, 1998; Golias et al., 1994; Marmin et al., 1998; Oden, 1973; Penman, 1988; Remacle et al., 1998; Rikabi et al., 1988).

Substituting the reconstruction operators [Eqs. (213) and (214)] in the error function [Eq. (215)], we obtain the local error function in terms of the cochains $\tilde{\Psi}^{h}$ and $\Phi^{b}$ :

$$
\xi(\mathbf{B}, \mathbf{H})=\xi\left(\mathbf{R}_{b}\left(\Phi^{b}\right), \mathbf{R}_{h}\left(\tilde{\Psi}^{h}\right)\right)
$$

Integrating $\xi$ on a space-time domain $\mathcal{D}$ we obtain a global error functional

$$
\mathcal{E}\left(\Phi^{b}, \tilde{\Psi}^{h}\right)=\int_{\mathcal{D}} \xi\left(\mathrm{R}_{b}\left(\Phi^{b}\right), \mathbf{R}_{h}\left(\tilde{\Psi}^{h}\right)\right)
$$

Since the cochain $\tilde{\Psi}^{h}$ is known, we can determine the optimal cochain $\Phi^{b}$ by means of the following optimization problem:

$$
\underline{\Phi}^{b}=\Phi^{b}: \min _{\Phi^{b}} \mathcal{E}\left(\Phi^{b}, \tilde{\Psi}^{h}\right)
$$

This procedure implicitly defines a link of the kind $\Phi^{b}=F_{\mu}\left(\tilde{\Psi}^{h}\right)$ and, therefore, establishes a discrete constitutive relation approximating the local constitutive relation.

Note that this approach to the discretization of a constitutive relation puts the two fields linked by the equation on a more equal level than does the previous strategy. There is, of course, still a direction of the link, going from the known cochain to the unknown one, but, in terms of the coefficients of the cochains involved, the link is no longer many-to-one but many-to-many. From a physical point of view this appears sound, considering that a constitutive relation should not be considered a cause-effect relationship in which a field is given, which fully determines another field, but instead must be viewed as 
a constraint which codetermines both fields. Note also that, in general, the minimization problem [Eq. (217)] takes place in space-time and not in space only. In fact, in a time-dependent problem, a minimization procedure must be performed at each step, for example, on the space-time domain constituted by the Cartesian product of the domain $D$ in space multiplied by the time step $\Delta t$, as follows:

$$
\mathcal{E}\left(\Phi^{b}, \tilde{\Psi}^{h}\right)=\int_{\Delta t} \int_{D} \xi\left(\mathbf{R}_{b}\left(\Phi^{b}\right), \mathbf{R}_{h}\left(\tilde{\Psi}^{h}\right)\right)
$$

The necessity of solving an optimization problem at each time step can greatly increase the computational cost of this strategy. In fact, the error-based approach was applied in the past mainly to static or quasistatic problems, or to frequency-domain problems (Albanese and Rubinacci, 1993). This is also because the required theoretical analyses and error functions were first given for these cases. However, the development of computing machines can quickly make attractive this kind of approach in alternative to the reconstructionprojection strategy for time-dependent problems also (Albanese et al., 1994; Albanese and Rubinacci, 1998).

\section{Edge Elements and Field Reconstruction}

The discretization strategies for constitutive relations that we have presented require the reconstruction of field functions on the basis of the unknowns of the discrete formulation of the problem. This appears at first as a familiar problem with function approximation. However, in our case, the starting data are cochains, that is, collections of global values on cells, not nodal samples of field functions. In other words, instead of a traditional nodal-based approximation problem, we must consider a problem of, cochain-based field function approximation (Mattiussi, 1997, 1998; Fig. 39). The two concepts coincide
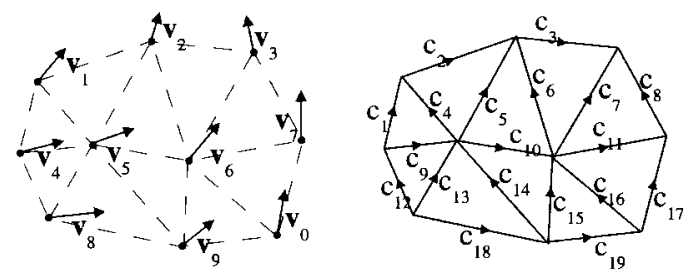

FIGURE 39. (Left) A nodal-based field function approximation is based on a set of local scalar or vector values defined on a grid of points. (Right) A cochain-based field function approximation takes instead as its starting point a $p$-cochain, that is, a set of global values associated with the oriented $p$-cells of the mesh. Here the case of ordinary 1-cochains on two-dimensional domains is considered. 
only for the case quantities associated with points, which, for theories having scalar global values, are represented in the continuous case by 0 -forms (i.e., by scalar field functions), and in the discrete case by scalar-valued 0 -cochains. Working only with unknowns associated with points, one can easily overlook the fact that the reconstruction is actually based on cochains, and this is what happens, for example, with a potential formulation of electrostatics. However, already in magnetostatics, one is faced with the fact that neither the fields nor the vector potential is associated with points. To use the traditional nodalbased tools of approximation theory in this case one is, therefore, forced to ignore the correct association of physical quantities with geometric objects, and consequently also to abandon any hope of complying with the structure of the field problem. The alternative is the introduction of new approximation tools tailored to the characteristics of cochains. In this sense it can be said that one needs to consider at least three-dimensional magnetostatics to start appreciating the true nature of the task constituted by the discretization of an electromagnetic problem.

In summary, an ordinary approximation problem asks: "Find on a given domain a scalar-valued or vector-valued function which approximates the data constituted by local scalar or vector values defined on a set of points." However, our approximation problem states: "Find on a given domain a $p$-form that approximates the data constituted by global values associated with the $p$-cells of a cell complex." In other words, we are requiring of the reconstructed $p$-form to have the given cochain as its projection onto the complex. A traditional approach to the solution of approximation problems within numerical methods is the selection of a set of shape functions. In our case, these are suitable forms $\sigma_{i}^{p}(\mathbf{r})$, that is, $p$-forms with the correct kind of orientation for the $p$-cochain one starts with, which can be used as a basis for the reconstruction, for example, in terms of a linear combination of them, as in

$$
\omega^{p}(\mathbf{r})=\sum_{i} a^{i} \sigma_{i}^{P}(\mathbf{r})
$$

The reconstruction must, of course, be uniquely determined (and, in fact, it must be a well-conditioned operation), and this is reflected in independence requirements of the shape functions. If instead of differential forms, we choose to work with the traditional tools of vector calculus, the entity to be approximated is a scalar or vector function; that is, a $p$-field defined in the domain. Correspondingly the forms $\sigma_{i}^{p}(\mathbf{r})$ become field functions $s_{i}^{p}(\mathbf{r})$ or $\mathbf{s}_{i}^{p}(\mathbf{r})$. From now on we will speak generically of shape functions, including in this definition differential forms and scalar and vector functions.

In general, the shape functions for an approximation problem are defined globally; that is, they are nonzero on the whole domain. In numerical methods 
for field problems it is often preferable to define shape functions of a local nature; that is, functions that are nonzero only within the domain constituted by a small number of adjacent cells. A class of shape functions, which complies with all the requirements listed so far, is that of the so-called edge elements. These are shape functions, which were introduced about 20 years ago in finite element practice (Ahagan et al., 1996; Albanese and Rubinacci, 1998; Webb, 1993; Jin, 1993). Edge elements are usually defined in terms of the kind of interelement discontinuities they permit. We will offer instead the following definition: an edge element is a shape function $\sigma^{p}$ defined in a domain subdivided by a cell complex, whose projection on the cell complex is an elementary $p$-cochain; that is, a cochain whose value is one on a particular $p$-cell $\tau_{p}^{i}$ and is zero on all other $p$-cells of the cell complex. In formulas,

$$
\int_{\tau_{p}^{i}} \sigma_{j}^{p}= \begin{cases}1 & \text { if } i=j \\ 0 & \text { if } i \neq j\end{cases}
$$

Note that Eq. (219) is a natural extension to generic geometric objects of the requirement traditionally imposed on the nodes of scalar shape functions. If the shape functions in the reconstruction [Eq. (218)] satisfy Eq. (219), they also satisfy automatically the property expressed by Eq. (210); that is, we have $P^{*} R_{*}=I$. To comply with the requirements of numerical methods, we ask also of this shape function to be nonzero only on a small neighborhood of $\tau_{p}^{i}$. We will call such a shape function an ordinary or a twisted (depending on the orientation of the corresponding cochain) p-edge element, to emphasize the correspondence to a particular oriented geometric object.

The aforementioned definition of edge elements is intended as a unifying definition in terms of the role they play in the discretization process, that of cochain-based field function approximation (their possible role as weight functions is discussed later, in the context of the finite element methods). Paralleling the reasons behind the introduction of the reference discretization strategy, this definition of the edge elements is not intended to give a sterile classification, but rather to help in testing existing elements for their consistency with this role, to be a guide for the development of new elements, and to assist in extending the application of edge elements to new fields. Our definition of edge elements may seem strange to edge elements practitioners also because such practitioners are accustomed to taking as their starting point the averaged components of the field to be reconstructed (be they tangent or normal to $p$-cells). A bit of reflection, however, reveals that the two ideas are perfectly equivalent, since multiplying the averaged field component by the extension of the cell, one obtains the global value associated with the cell, whereas the fact that only the averaged tangential or normal component (to accommodate internal or 

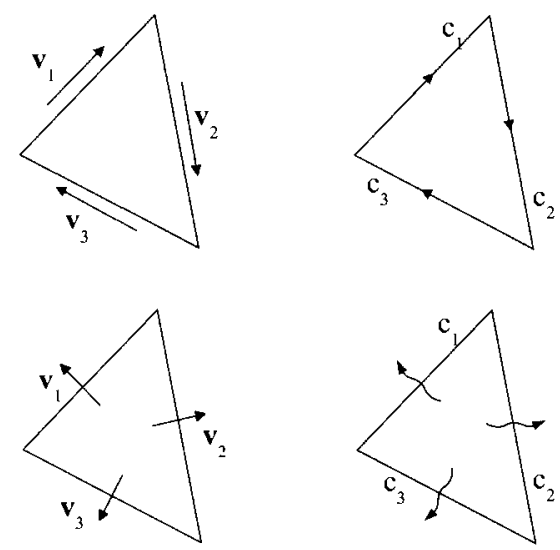

Figure 40. (Left Column) Edge elements are usually considered as based on the averaged components of the field tangent to the cells or normal to them. This is, however, equivalent to considering the corresponding global values associated with internally or externally oriented cells, respectively (right column). Edge elements for two-dimensional problems are considered here. Note that wavy arrows represent external orientation of geometric objects and not the presence of vectors associated with them.

external orientation, respectively) is considered ensures that the field quantities contain no more information than the global value (Fig. 40).

From our definition of edge elements it follows that given a cochain $\mathbf{c}^{p}$ on a cell complex $K$ and a set of edge elements $\left\{\sigma_{i}^{p}\right\}$, one for each $p$-cell of $K$, we can construct a field function in the domain $|K|$ as a linear combination of the kind of Eq. (218), but this time with the coefficients $c^{i}$ that are the values taken by the cochain to be reconstructed on the $p$-cells of the complex; that is, the vector that represents the cochain with respect to the natural basis for cochains [Eq. (106)].

The simple requirement of having an elementary cochain as their projection does not uniquely determine edge elements. One can indeed find a multitude of shape functions that comply with this requirement, and in particular with Eq. (219). For this reason, one tries, in selecting edge elements for a problem, to satisfy other properties also, in particular those related to the accuracy of the reconstruction and, therefore, of the computation. These include, for example, the presence of the polynomial terms up to a given order in the reconstructed functions or in a transformation thereof (Sun et al., 1995). Note that in some cases a certain number of missing terms in the reconstructed function can be dispensed with, with a proper placement of the meshes (Mattiussi, 1997).

In this quest for a higher-order edge element, one may end up defining shape functions that resemble edge elements but, according to our definition, 


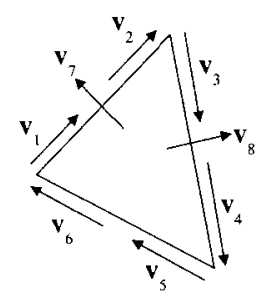

Figure 41. Anomalous edge elements mix internal and external orientations and associate multiple quantities with a single geometric object, which thus violates the principles of the association of physical quantities with geometric objects.

are not. This is the case for a number of the so-called vector elements that have been proposed to improve the behavior of the first generation of edge elements (Cendes, 1991; Sun et al., 1995). Let us follow the path that leads to the introduction of these elements. We consider 1 -edge elements (i.e., elements whose projections are 1-cochains) for two-dimensional problems, and we represent them with the degrees of freedom they associate with a triangle (Fig. 40). It is apparent that edge elements of this kind permit the reconstruction of 1-fields on the primary and secondary meshes. These elements are characterized, however, by a small number of degrees of freedom, and, therefore, by a small number of terms in the approximating polynomials. This translates into a slow rate of convergence for the methods in which they are employed (Sun et al., 1995). To circumvent this problem, one would naturally increase the number of degrees of freedom associated with each edge element. Figure 41 shows the result, in terms of degrees of freedom, for a popular vector element derived following this idea. It is apparent that these elements mix internal and external orientations and associate multiple quantities with a single geometric object. Thus, reconstruction based on these kinds of elements violates two of the fundamental principles of the association of physical quantities with geometric objects.

Since they do not comply with our definition of edge elements, we will call these kinds of elements anomalous edge elements. Anomalous edge elements show that not every non-nodal-based shape function is an edge element. There is no doubt, however, that the introduction of such elements was dictated by the necessity to overcome some real problems. Let us, therefore, try to bring these elements back within our definition of edge element. To this end, we must first ensure that the number of geometric objects appearing in the element equals the number of instances of the physical quantity that we want to associate with it (i.e., the number of degrees of freedom of the element). We do this by adding to the original element a suitable number of geometric objects of the correct dimension and orientation. In this way we end up with an element with the 


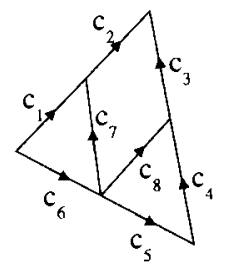

FIGURE 42. Anomalous edge elements can be brought back to conformity with the prescriptions deriving from the structural analysis of physical field theories, by introducing additional geometric objects.

same number of degrees of freedom as that of the original anomalous element, except that each instance of the physical quantity is now associated with a distinct geometric object, and the kind of orientation is the same for all the cells intervening in the reconstruction. For example, in the case of Figure 41 the quantity under consideration is associated with internally oriented cells and, therefore, the new geometric objects are primary 1-cells; Figure 42 shows a possible result of the process just described.

Figure 42 shows also that the support of an edge element can be composed by the assembly of several $p$-cells of the cell complex. This determines an interpolation domain which is the union of several $n$-cells of the corresponding cell complex (where $n$ is the dimension of the domain). In fact, this assembly of the $p$-cells belonging to several neighboring $n$-cells of the cell complex is the easiest way to increase the number of terms in the resulting interpolating polynomial while preserving the local nature of the interpolation process. It appears, therefore, that the recourse to field function reconstruction for the discretization of the constitutive equations implies, besides the definition of the primary and secondary meshes, the introduction of an additional discretization structure for the geometry. This additional discretization structure is composed of by what we will call the elements, which are the domains on which separate approximation problems are solved to reconstruct the field function starting from the cochain coefficients. For example, for the reconstruction of an ordinary (or a twisted) $p$-field we consider within each element the ordinary (or twisted) $p$-cells and use the physical quantities associated with these cells (and only these) to build the field function approximation which holds true within the element. We will call element mesh the structure determined by the elements (Fig. 43). Note that an element mesh is required for each field to be reconstructed. Note also that even if usually each element of the mesh is constituted by the union of a small number of $n$-cells of the primary or secondary mesh, this is not mandatory. One could in fact conceive of a separately defined element mesh to accommodate this process, which can be itself a cell complex 


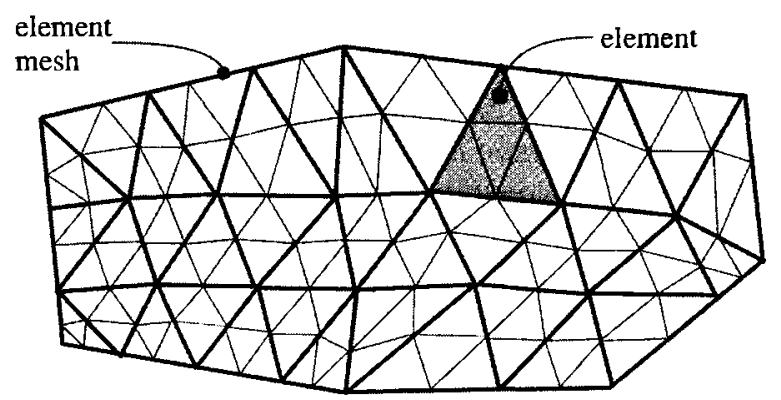

FIGURE 43. The recourse to field reconstruction for the discretization of the constitutive relations requires the definition of an additional geometric structure, in addition to the primary and secondary meshes, namely, an element mesh for each field to be reconstructed. Here each object of the new mesh is obtained as the union of cells of the primary or secondary mesh, but more general geometric structures not based on the two preexisting meshes can be used as well.

or not (e.g., different elements could overlap), or, as in the case of meshless methods (Belytschko et al., 1996), no element mesh at all.

Finally, we will make some scattered comments on edge elements and the operation of reconstruction in general. First, we should mention that edge elements alone do not guarantee that a discretization complies with the results of the analysis of the structure of physical theories. Given a cochain, edge elements reconstruct a field which complies with that cochain, in the sense that the latter is a projection of the former. However, if the cochain we start with is a nonphysical one, in that, for example, it does not satisfy the topological laws of our theory, we cannot ask the reconstructed field to do so. Hence, only a proper formulation of the field problem, along with the use of edge elements in the reconstruction of field functions, guarantees the physical soundness of the solution (Mur, 1994). Next, note that within the approach presented in this section, shape functions are not used to obtain a continuous field defined on the whole domain, but only as a step in the realization of the discretization strategies for constitutive relations. From this point of view, they are a tool which is used temporarily in a phase of the discretization process after which they are discarded. Of course, one must not be careless in using this tool. In particular, one must consider the fact that the discontinuities in the properties of materials usually produce corresponding discontinuities in some of the field functions. Therefore, to properly approximate the constitutive equations in elements containing material discontinuities, one sees here the necessityfor the first time - of taking into account these material discontinuities and of making them coincide with element boundaries, so that discontinuities of the field functions can be modeled by the reconstruction process. We emphasize 
"for the first time" because the discrete rendering of topological laws, as presented previously, is not disturbed by the presence of material discontinuities, since topological laws do not depend on material parameters. In fact, when one is dealing with global quantities only, the very concept of field function continuity is meaningless. Note also that the reconstruction is instrumental to the constitutive relations discretization, which implies that we do not ask the reconstructed fields to satisfy the topological laws (in local, differential form), since these are imposed only (in global form) at the cell-complex level.

\section{B. Finite Difference Methods}

We now deal with the comparison of existing methods with the reference discretization strategy just detailed, starting with the finite difference (FD) methods. We should mention in advance that in this presentation of the methods the references typically cited are not founding papers but preferably survey works including extensive bibliographies.

The classical FD approach to the discretization of field problems is based on the use of FD formulas to approximate locally the derivatives entering the expression of differential operators. A structured grid of points is defined firstusually a very regular one - and a local field quantity is attached to each point. Then, for each of these points the differential operators appearing in the problem's equations are given a discrete expression by means of the aforementioned FD formulas. Given the absence of any reference to the association of physical quantities with geometric objects other than points, one can hardly expect such an approach to yield results consistent with those of the analysis developed thus far. This was indeed the case for the first attempts to give an FD formulation for electromagnetic problems. These attempts resulted in methods that have practically nothing in common with the reference method developed in the previous section. The first notable exception was the well-known finite difference time-domain method, the analysis of which offers some interesting results.

\section{The Finite Difference Time-Domain Method}

Consider an electromagnetic problem with constitutive equations $\mathbf{B}=\mu \mathbf{H}$ and $\mathbf{D}=\varepsilon \mathbf{E}$, and, for simplicity, the absence of electric losses. To solve this problem, the finite difference time-domain (FDTD) method starts by defining within the space-time domain of the problem two dual orthogonal Cartesian grids. These subdivide the space domain into parallelepipeds having size $\Delta x, \Delta y$, and $\Delta z$. The time domain is subdivided into time steps of size $\Delta t$. The primary and secondary grids are staggered by a half step in both space and time. To preserve the distinction emphasized thus far between primary and secondary 


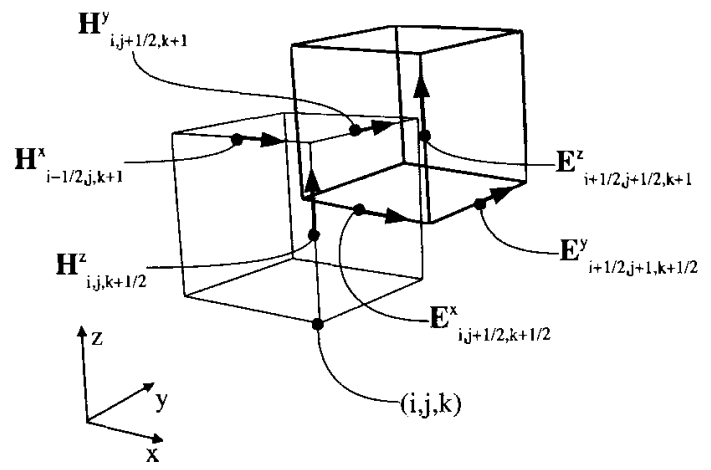

FIGURE 44. The FDTD method makes use of two dual orthogonal grids staggered by a half step in space and in time. The variables appearing in the time-stepping formulas are the field components of $\mathbf{E}$ and $\mathbf{H}$ tangent to the grid edges and evaluated in the midpoint of each edge.

geometric objects, and the corresponding one between the related quantities, we will write $\widetilde{\Delta x}, \widetilde{\Delta y}, \widetilde{\Delta z}$, and $\widetilde{\Delta t}$ for the discretization steps of the secondary grid, even if in the FDTD method these quantities coincide numerically with the primary ones. The variables used within the method are the $x, y$, and $z$ components of the electric and magnetic fields $\mathbf{E}$ and $\mathbf{H}$, which are attached to the midpoint of the grid edges, and the local values of the material properties at the same locations. The nodes and the associated quantities are individuated by integral or half-integral indexes; for example, $E_{(i, j+1 / 2, k+1 / 2), n+1 / 2}^{x}$ stands for $E^{x}(i \Delta x,(j+1 / 2) \Delta y,(k+1 / 2) \Delta z ;(n+1 / 2) \Delta t)$ (Fig. 44).

With this symbolism, the FDTD time-stepping formulas are, for the time stepping of $H^{x}$,

$$
\begin{aligned}
\Delta y & \Delta z \mu_{(i+1 / 2, j, k)}\left(H_{(i+1 / 2, j, k), n+1}^{x}-H_{(i+1 / 2, j, k), n}^{x}\right) \\
= & \Delta y \Delta t\left(E_{(i+1 / 2, j, k+1 / 2), n+1 / 2}^{y}-E_{(i+1 / 2, j, k-1 / 2), n+1 / 2}^{y}\right) \\
& -\Delta z \Delta t\left(E_{(i+1 / 2, j+1 / 2, k), n+1 / 2}^{z}-E_{(i+1 / 2, j-1 / 2, k), n+1 / 2}^{z}\right)
\end{aligned}
$$

and, for the time stepping of $E^{x}$,

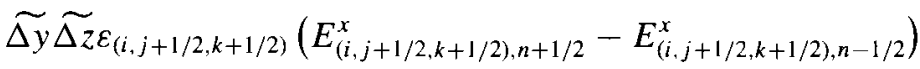

$$
\begin{aligned}
& =-\widetilde{\Delta y} \widetilde{\Delta t}\left(H_{(i, j+1 / 2, k+1), n}^{y}-H_{(i, j+1 / 2, k), n}^{y}\right) \\
& +\widetilde{\Delta z \widetilde{\Delta t}}\left(H_{(i, j+1, k+1 / 2), n}^{2}-H_{(i, j, k+1 / 2), n}^{2}\right)
\end{aligned}
$$

Analogous relations hold for the time stepping of the other components of E and H (Kunz and Luebbers, 1993; Taflove, 1995). Note that the method seemingly does not make use of global physical quantities, resorting instead 
to nodal values of local vector quantities. We can, however, observe that each of the field components appearing in these formulas can be considered the ratio of the global quantity associated with a cell and the extension of the cell itself. Interpreting the local field components in this way, that is, as averaged field components with respect to spacelike and space-time 2-cells, and remembering that from the local constitutive equations it follows that $B_{(i+1 / 2, j, k), n}^{x}=\mu_{(i+1 / 2, j, k)} H_{(i+1 / 2, j, k), n}^{x}$ and $D_{(i, j+1 / 2, k+1 / 2), n+1 / 2}^{x}=\varepsilon_{(i, j+1 / 2, k+1 / 2)}$ $E_{\{i, j+1 / 2, k+1 / 2), n+1 / 2}^{x}$, we can write

$$
\begin{aligned}
\Delta y \Delta z \mu_{(i+1 / 2, j, k)} H_{(i+1 / 2, j, k), n+1}^{x} & =\phi_{(i+1 / 2, j, k), n+1}^{b_{x}} \\
\Delta y \Delta t E_{(i+1 / 2, j, k \pm 1 / 2), n+1 / 2}^{y} & =\phi_{(i+1 / 2, j, k \pm 1 / 2), n+1 / 2}^{e_{y}} \\
\Delta z \Delta t E_{(i+1 / 2, j \pm 1 / 2, k), n+1 / 2}^{z} & =\phi_{(i+1 / 2, j \pm 1 / 2, k), n+1 / 2}^{e_{2}}
\end{aligned}
$$

and

$$
\begin{aligned}
\widetilde{\Delta y \widetilde{\Delta z} \varepsilon_{(i, j+1 / 2, k+1 / 2)} E_{(i, j+1 / 2, k+1 / 2), n+1 / 2}^{x}} & =\psi_{(i, j+1 / 2, k+1 / 2), n+1 / 2}^{d_{x}} \\
\widetilde{\Delta y} \widetilde{\Delta t} H_{(i, j+1 / 2, k+1), n}^{y} & =\psi_{(i, j+1 / 2, k+1), n}^{h_{y}} \\
\widetilde{\Delta z} \widetilde{\Delta t} H_{(i, j+1, k+1 / 2), n}^{z} & =\psi_{(i, j+1, k+1 / 2), n}^{h_{i}}
\end{aligned}
$$

With these definitions the FDTD method can be described as working in terms of global quantities. In particular, the FDTD time-stepping formula for $H^{x}$ [Eq. (220)] becomes the following time-stepping formula for $\phi^{b_{x}}$,

$$
\begin{aligned}
\phi_{(i+1 / 2, j, k), n+1}^{b_{x}}= & \phi_{(i+1 / 2, j, k), n}^{b_{x}}+\phi_{(i+1 / 2, j, k+1 / 2), n+1 / 2}^{e_{y}}-\phi_{(i+1 / 2, j, k-1 / 2), n+1 / 2}^{e_{y}} \\
& -\phi_{(i+1 / 2, j+1 / 2, k), n+1 / 2}^{e_{z}}+\phi_{(i+1 / 2, j-1 / 2, k), n+1 / 2}^{e_{z}}
\end{aligned}
$$

and the FDTD time-stepping formula for $E^{x}[\mathrm{Eq} .(221)]$ becomes

$$
\begin{aligned}
\psi_{(i, j+1 / 2, k+1 / 2), n+1 / 2}^{d_{x}}= & \psi_{(i, j+1 / 2, k+1 / 2), n-1 / 2}^{d_{x}}-\psi_{(i, j+1 / 2, k+1), n}^{h_{y}}+\psi_{(i, j+1 / 2, k), n}^{h_{y}} \\
& +\psi_{(i, j+1, k+1 / 2), n}^{h_{z}}-\psi_{(i, j, k+1 / 2), n}^{h_{z}}
\end{aligned}
$$

Comparing Eqs. (228) and (229) with the time-stepping formulas of the reference discretization method [Eqs. (188) and (190)], we recognize that the former are a particular case of the latter. The signs of the $\phi^{e}$ and $\psi^{h}$ terms on the right sides of Eqs. (228) and (229) correspond to the incidence numbers appearing in the reference formulas.

From Eq. (222) we see that the following relation holds true:

$$
H_{(i+1 / 2, j, k), n}^{x}=\frac{1}{\mu_{(i+1 / 2, j, k)}} \frac{1}{\Delta y \Delta z} \phi_{(i+1 / 2, j, k), n}^{b_{x}}
$$


Conversely, from the equation corresponding to Eq. (226) for the case of the $x$ component, we determine

$$
H_{(i+1 / 2, j, k), n}^{x}=\frac{1}{\widetilde{\Delta x} \widetilde{\Delta t}} \psi_{(i+1 / 2, j, k), n}^{h_{x}}
$$

Comparing Eqs. (230) and (231), we obtain

$$
\phi_{(i+1 / 2, j, k), n}^{b_{x}}=\mu_{(i+1 / 2, j, k)} \frac{\Delta y \Delta z}{\widetilde{\Delta x} \widetilde{\Delta t}} \psi_{(i+1 / 2, j, k), n}^{h_{x}}
$$

This is the constitutive equation linking $\phi^{b_{x}}$ to $\psi^{h_{x}}$. The same procedure, applied to Eqs. (225) and (223), gives

$$
\psi_{(i, j+1 / 2, k+1 / 2), n+1 / 2}^{d_{x}}=\varepsilon_{(i, j+1 / 2, k+1 / 2)} \frac{\widetilde{\Delta y} \widetilde{\Delta z}}{\Delta x \Delta t} \phi_{(i, j+1 / 2, k+1 / 2), n+1 / 2}^{e_{x}}
$$

Equations (232) and (233) are clearly discrete constitutive equations of the simplest type, like Eq. (205), obtained by extending the local constitutive equations $\mathbf{B}=\mu \mathbf{H}$ and $\mathbf{D}=\varepsilon \mathbf{E}$ and exploiting the planarity, regularity, and orthogonality of cells in the meshes adopted by the FDTD method. This is even more clear when we write Eqs. (232) and (233) as follows:

$$
\begin{aligned}
& \frac{\phi_{(i+1 / 2, j, k), n}^{b_{x}}}{\Delta y \Delta z}=\mu_{(i+1 / 2, j, k)} \frac{\psi_{(i+1 / 2, j, k), n}^{h_{x}}}{\widetilde{\Delta x \widetilde{\Delta t}}} \\
& \frac{\psi_{(i, j+1 / 2, k+1 / 2), n+1 / 2}^{d_{x}}}{\widetilde{\Delta y \widetilde{\Delta z}}}=\varepsilon_{(i, j+1 / 2, k+1 / 2)} \frac{\phi_{(i, j+1 / 2, k+1 / 2), n+1 / 2}^{e_{x}}}{\Delta x \Delta t}
\end{aligned}
$$

Therefore, we can affirm that the FDTD method implicitly uses discretization strategy 1 for the discretization of constitutive relations. Substituting these discrete constitutive equations, or their inverse, in the time-stepping formulas [Eqs. (228) and (229)], we obtain time-stepping formulas in terms of two global variables only. In particular, the formulas in terms of $\phi^{b}$ and $\psi^{d}$ are

$$
\begin{aligned}
\phi_{(i+1 / 2, j, k), n+1}^{b_{x}} & \phi_{(i+1 / 2, j, k), n}^{b_{x}}+\frac{\Delta x \Delta t}{\widetilde{\Delta y \widetilde{\Delta z}}}\left(\frac{1}{\varepsilon_{(i+1 / 2, j, k+1 / 2)}} \psi_{(i+1 / 2, j, k+1 / 2), n+1 / 2}^{d_{x}}\right. \\
& -\frac{1}{\varepsilon_{(i+1 / 2, j, k-1 / 2)}} \psi_{(i+1 / 2, j, k-1 / 2), n+1 / 2}^{d_{x}}-\frac{1}{\varepsilon_{(i+1 / 2, j+1 / 2, k)}} \psi_{(i+1 / 2, j+1 / 2, k), n+1 / 2}^{d_{x}} \\
& \left.+\frac{1}{\varepsilon_{(i+1 / 2, j-1 / 2, k)}} \psi_{(i+1 / 2, j-1 / 2, k), n+1 / 2}^{d_{x}}\right)
\end{aligned}
$$


and

$$
\begin{aligned}
& \psi_{(i, j+1 / 2, k+1 / 2), n+1 / 2}^{d_{x}} \\
& =\psi_{(i, j+1 / 2, k+1 / 2), n-1 / 2}^{d_{x}}-\frac{\widetilde{\Delta x} \widetilde{\Delta t}}{\Delta y \Delta z}\left(\frac{1}{\mu_{(i, j+1 / 2, k+1)}} \phi_{(i, j+1 / 2, k+1), n}^{b_{x}}\right. \\
& \quad+\frac{1}{\mu_{(i, j+1 / 2, k)}} \phi_{(i, j+1 / 2, k), n}^{b_{x}}+\frac{1}{\mu_{(i, j+1, k+1 / 2)}} \phi_{(i, j+1, k+1 / 2), n}^{b_{x}} \\
& \left.\quad-\frac{1}{\mu_{(i, j, k+1 / 2)}} \phi_{(i, j, k+1 / 2), n}^{b_{r}}\right)
\end{aligned}
$$

Analogous formulas can be written for the other components.

It is interesting to consider also the case of lossy materials, in which case Eq. (221) becomes (Taflove, 1995)

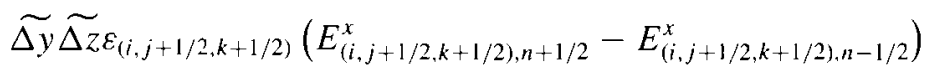

$$
\begin{aligned}
& =-\widetilde{\Delta y} \widetilde{\Delta t}\left(H_{(i, j+1 / 2, k+1), n}^{y}-H_{(i, j+1 / 2, k), n}^{y}\right) \\
& +\widetilde{\Delta z \widetilde{\Delta t}}\left(H_{(i, j+1, k+1 / 2), n}^{z}-H_{(i, j, k+1 / 2), n}^{z}\right) \\
& +\widetilde{\Delta y} \widetilde{\Delta z} \widetilde{\Delta t} \sigma_{(i, j+1 / 2, k+1 / 2)}\left(\frac{E_{(i, j+1 / 2, k+1 / 2), n+1 / 2}^{x}+E_{(i, j+1 / 2, k+1 / 2), n-1 / 2}^{x}}{2}\right)
\end{aligned}
$$

The new term with respect to Eq. (221) represents the charge flowing through a surface $(\widetilde{\Delta y} \widetilde{\Delta z})$ during a time interval $\widetilde{\Delta t}$, and can, therefore, be written as

$$
\begin{aligned}
& \widetilde{\Delta y} \widetilde{\Delta z} \widetilde{\Delta t} \sigma_{(i, j+1 / 2, k+1 / 2)}\left(\frac{E_{(i, j+1 / 2, k+1 / 2), n+1 / 2}^{x}+E_{(i, j+1 / 2, k+1 / 2), n-1 / 2}^{x}}{2}\right) \\
& =Q_{(i, j+1 / 2, k+1 / 2), n}^{j_{i}}
\end{aligned}
$$

Thus, with the new term, the lossless time-stepping formula [Eq. (229)] becomes

$$
\begin{aligned}
\psi_{(i, j+1 / 2, k+1 / 2), n+1 / 2}^{d_{r}}= & \psi_{(i, j+1 / 2, k+1 / 2), n-1 / 2}^{d_{x}}-\psi_{(i, j+1 / 2, k+1), n}^{h_{v}}+\psi_{(i, j+1 / 2, k), n}^{h_{v}} \\
& +\psi_{(i, j+1, k+1 / 2), n}^{h_{z}}-\psi_{(i, j, k+1 / 2), n}^{h_{z}}+Q_{(i, j+1 / 2, k+1 / 2), n}^{h_{x}}
\end{aligned}
$$


and we have the new discrete constitutive equation

$$
\begin{aligned}
& Q_{(i, j+1 / 2, k+1 / 2), n}^{j_{x}} \\
& =\frac{\widetilde{\Delta y} \widetilde{\Delta z} \widetilde{\Delta t}}{\Delta x \Delta t} \sigma_{(i, j+1 / 2, k+1 / 2)}\left(\frac{\phi_{(i, j+1 / 2, k+1 / 2), n+1 / 2}^{e_{x}}+\phi_{(i, j+1 / 2, k+1 / 2), n-1 / 2}^{e_{x}}}{2}\right)
\end{aligned}
$$

which can also be written as

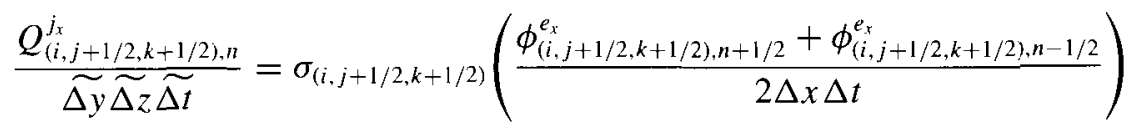

Note that, contrary to Eqs. (232) and (233), which are one-to-one discrete constitutive equations, Eq. (241) links $Q^{j_{x}}$ to two distinct values of $\phi^{e_{x}}$, which correspond to two consecutive primary time intervals. This can be explained by considering that the space-time volume to which $Q^{j_{r}}$ is associated spans a secondary time interval $\widetilde{\Delta t}$, which is only half covered by a primary time interval $\Delta t$. From this, it is desirable to involve at least two instances of $\phi^{e_{x}}$ in the determination of a single instance of $Q^{j_{x}}$. So that we can actually obtain from Eq. (240) a time-stepping formula, the last term must be expressed in terms of electric fluxes only. To this end, we invert the discrete constitutive relation [Eq. (233)], obtaining

$$
\phi_{(i, j+1 / 2, k+1 / 2), n+1 / 2}^{e_{x}}=\frac{1}{\varepsilon_{(i, j+1 / 2, k+1 / 2)}} \frac{\Delta x \Delta t}{\widetilde{\Delta y} \widetilde{\Delta z}} \psi_{(i, j+1 / 2, k+1 / 2), n+1 / 2}^{d_{x}}
$$

so that $Q^{j_{x}}$ in the time-stepping formula [Eq. (240)] can be expressed as

$$
\begin{aligned}
& Q_{(i, j+1 / 2, k+1 / 2), n}^{j_{x}} \\
& \quad=\widetilde{\Delta t} \frac{\sigma_{(i, j+1 / 2, k+1 / 2)}}{\varepsilon_{(i, j+1 / 2, k+1 / 2)}}\left(\frac{\psi_{(i, j+1 / 2, k+1 / 2), n+1 / 2}^{d_{x}}+\psi_{(i, j+1 / 2, k+1 / 2), n-1 / 2}^{d_{x}}}{2}\right)
\end{aligned}
$$

Note how this relation involves two constitutive links: one going from primary to secondary quantities, and one going in the opposite direction (Fig. 37).

In summary, with the interpretation of physical quantities suggested in Eqs. (222) through (224), (225) through (227), and (239), the FDTD method appears to adopt a discretization strategy fully consistent with the prescriptions of the analysis of the structure of physical field theories, both in the discretization of the geometry and in the association of global physical quantities to 
space-time geometric objects. The FDTD method thus appears as a particular instance of the reference discretization method presented previously. The time marching is performed by means of the truly topological time-stepping formulas [Eqs. (228) and (240)], supplemented by the discrete constitutive relations [Eqs. (232), (233), and (241)]. Note that to determine the discrete constitutive equations, the constitutive relations are implicitly subjected to the simplest of the three kinds of discretization strategies considered previously. This leads one to expect that if this consequence of the original, intuitive approach which led to the FDTD method is not properly recognized beforehand, the efforts trying to extend the method to higher orders in space and time, or to nonorthogonal and unstructured meshes, are bound to produce mediocre results or to meet with severe difficulties. [For a more detailed analysis and a four-dimensional interpretation of the physical quantities and topological time stepping within the FDTD method see Mattiussi (2001).]

\section{The Support Operator Method}

The support operator method (SOM; Hyman and Shashkov, 1997, 1999; Shashkov, 1996; Shashkov and Steinberg, 1995) is an FD technique that permits the derivation of discrete approximations to differential operators, which preserve some properties of the original continuous mathematical model within which the operators to be approximated appear. In particular, the focus is on the simultaneous preservation of some integral identity that is used in writing a topological law in continuous terms, and of some adjointness relation between pairs of topological statements that face each other in the factorization diagram of the corresponding physical theory. Given this emphasis on integral relations, it is instructive to compare this approach with that of the reference discretization strategy.

The discretization of geometry adopted by the SOM is typical of FD methods in that a sufficiently well-behaved set of nodes is considered within the domain in space. For example, in two dimensions it is assumed that by properly joining these nodes one can construct at least a logically rectangular grid; that is, one that is homeomorphic to an actual rectangular grid (Shashkov, 1996). This implies that the resulting grid is, in fact, a cell complex, since it derives from the topological distortion of a subdivision of a domain in simple rectangular cells. Therefore, in addition to the 0-cells constituted by the original set of nodes, sets of $p$-cells with $p$ up to the dimension of the domain are implicitly defined. This constitutes the primary mesh. The SOM does not make explicit use of a secondary mesh.

The variables used by the method are the field components perpendicular or tangential to the cells, and associated with the centers of the cells (which, of course, in the case of the nodes are the nodes themselves). As in the case of the FDTD method, this opens the door to their interpretation as representatives of 
global physical quantities. In fact, apart from nodal quantities, these components appear in the method's formulas multiplied by the extension of the corresponding cell. Therefore, if we think of these variables as averaged values of the field over the cell, their products correspond to global quantities.

Up to this point, we have merely described some general premises of FD discretization. It is in the discretization of the field equations that the SOM differentiates itself from a classical FD approach. Instead of discretizing separately the differential operators appearing in the field equations, the SOM selects one of the differential operators to play a privileged role in the discretization. This is called the prime operator. The prime operator is discretized with an FD approach; that is, by substituting its derivatives with FD approximations. However, during this process one must try to preserve as much as possible the integral properties of the prime operator. For example, if the prime operator is a divergence, a discrete version of Gauss's divergence theorem must be applied to the discretized operator, which in this case is designated as DIV.

The other differential operators that appear in the field problem are then considered for discretization. These are related to the prime operator by some integral relation. We know from our previous analysis that these amount substantially to the continuous counterparts of two properties of the coboundary operator: the fact that $\delta \delta=0$ (from which the properties such as $d d=0$, curl $\operatorname{grad}=0$, and div curl $=0$ are derived), and the adjointness (with respect to a suitable bilinear form, which puts in duality the corresponding cochains' spaces) of the coboundary acting from ordinary $p$-cochains to produce ordinary $(p+1)$-cochains on the primary mesh, with the coboundary acting on twisted $(n-(p+1))$-cochains to give twisted $(n-p)$-cochains on the dual secondary mesh. For example, if the primary operator is a divergence, the corresponding integral relation is

$$
\int_{V} \varphi \operatorname{div} \mathbf{A}+\int_{V} \mathbf{A} \cdot \operatorname{grad} \varphi=\int_{\partial V} \varphi(\mathbf{A} \cdot \mathbf{n})
$$

In this case, to discretize the gradient operator, the SOM puts in duality the discrete spaces of the variables by means of a suitable inner product and enforces a discrete counterpart of this relation. The resulting discrete operator is marked in some way, to signify its being a derived operator instead of a prime operator. For example, if the divergence is adopted as a prime operator, and Eq. (245) is used to obtain a discrete counterpart of the gradient, the resulting discrete operator is denoted with $\overline{\text { GRAD. }}$.

We have not yet spoken of the constitutive links. The SOM does not adopt a separate discretization of these terms but includes instead this task in the discretization of some differential operator appearing in the field equations. Therefore, the SOM produces a discretized compound operator in place of a separate discrete constitutive operator. For example, the operators ${ }_{\varepsilon} \operatorname{curl}_{\mu} \stackrel{\text { def }}{=} \frac{1}{\varepsilon} \operatorname{curl} \frac{1}{\mu}$ 
and $\operatorname{div}^{\varepsilon} \stackrel{\text { def }}{=} \operatorname{div} \varepsilon$ are defined, and they are discretized with the same procedure adopted for the purely differential operators. The only difference is that a bilinear form, which includes the constitutive links, is used to put in duality the spaces of discrete variables prior to discretization. Hence, three types of discrete operators result: a first class of operators determined from prime differential operators, denoted with GRAD, CURL, and DIV; a second class of operators determined as derived discrete operators, denoted with $\overline{\text { GRAD }}, \overline{\mathrm{CURL}}$, and $\overline{\mathrm{DIV}}$; and a third class of operators determined as derived operators from compound differential operators, denoted, for example, with $\overline{\mathrm{DIV}}^{\varepsilon}$ and ${ }_{\varepsilon} \overline{\mathrm{CURL}}_{\mu}$.

Finally, the derivatives with respect to time that remain in the semidiscretized model when the differential operators have been substituted by their discrete counterparts are discretized with the traditional approaches; that is, approximating the time derivative with an FD formula. In particular the standard leapfrog method is suggested for this task.

Examining the workings of the SOM, we can see that the method recognizes the necessity to take into consideration a number of structural properties of the field problem. However, this is done when the problem itself has already been modeled in continuous terms (i.e., the branch on the right has been selected in Fig. 1). Therefore, properties such as quantity conservation and the adjointness of operators, which can be easily expressed and automatically enforced in discrete terms by adopting the discrete mathematical model of the reference strategy, are instead considered first at the continuous level and only subsequently enforced in a discrete setting. For this reason, the SOM appears to take a long detour to enforce properties that are automatically satisfied by using the approach based on the structure of physical theories. Moreover, the unique discrete operator for the representation of topological laws constituted by the coboundary operator and the related topological time-stepping process are not considered, and a separate discretization in space and in time is performed. In this sense, the SOM is representative of the task thus constituted and the possible pitfalls implied by the search for a structurally sound discretization strategy which takes as its starting point the continuous mathematical model.

\section{Beyond the FDTD Method}

The preceding analysis shows that the discretization strategy adopted by the FDTD method is a physically sound one. Moreover, the method is easy to understand and to implement, at least for simple materials. All this makes FDTD a very successful method. This success has logically led to many efforts focused on the removal of its limitations. These lie mainly in the scarce flexibility of its orthogonal grids in modeling complex geometries, and in the low order of accuracy of the method, both in space and in time. Consequently 
FDTD extensions to nonorthogonal or unstructured meshes, and to formulas having higher accuracy in space and time, have been presented (Taflove, 1998). Given the analysis presented in this work, and equipped with the conceptual tool represented by the reference discretization strategy, we know in what direction to look for an extension of FDTD capable of preserving its favorable qualities. The rationale can be stated as follows: "Keep two space-time cell complexes as meshes, keep the topological time stepping, and improve the discretization of the constitutive relations." Unfortunately, the classical FD approach says instead: "Use an expression of differential operators in generic curvilinear coordinates and increase the accuracy of the approximation of derivatives appearing in the partial differential equation." However, this does not lend itself to an easy generalization beyond logically rectangular grids. Moreover, since the derivatives appear in the equations as a consequence of the local representation of the topological operators, a brute-force approach to increasing the accuracy of their approximation, be they expressed in Cartesian coordinates or in curvilinear coordinates, is likely to lead to time-stepping formulas that cannot be considered as derived from a coboundary operator. We will, therefore, neglect the analysis of the extensions of FDTD based on local viewpoints, such as the classical FD approach, and strategies based on ideas borrowed from differential geometry, which make use, for example, of covariant and contravariant local basis vectors to express the differential operators on nonorthogonal grids (Taflove, 1998). We will look instead directly to methods which preserve the focus on the discrete nature of topological laws-namely, finite volume methods.

\section{Finite Volume Methods}

In general, we can say that a numerical method is a finite volume (FV) method if, to discretize the field equations of a problem, it subdivides the problem domain into cells and writes the field equations in integral form on these cells. If we accept this definition, we see at once that the FV approach is very similar to that advocated by the reference method. However, the adoption of an integral approach alone does not ensure that all the requirements of the physical approach to the discretization are recognized and implemented. For example, one could write an integral statement in which topological and constitutive links are mixed, thus missing a fundamental distinction. Moreover, usually the discretization produced by the integral statements of the FV method does not include the time variable, which is instead subjected to a separate discretization. This opens the door to the possibility of time-stepping formulas that cannot be derived in a natural way from a space-time coboundary operator. From this point of view, the first FV method for time-dependent electromagnetic 
problems that we will examine will turn out to be well behaved, in that it can be interpreted as a particular case of the reference discretization strategy.

\section{The Discrete Surface Integral Method}

The discrete surface integral (DSI) method was suggested by Madsen (1995) for the solution of time-dependent electromagnetic problems on domains discretized by using unstructured grids. The method was first presented for the case of lossless, linear, isotropic materials, but it can easily be extended to lossy materials (Taflove, 1998) and to more complex electric and magnetic constitutive relations.

For the discretization of the domain in space the method requires two dual meshes constructed exactly like those of the reference strategy (Fig. 33), but for the fact that within the DSI method there is no mention of the distinction between external and internal orientations. To simplify things with respect to a generic cell complex, we will assume that all 1-cells are straight lines and all 2-cells are planar. The variables used as unknowns by the method are at first sight not global ones but are instead field quantities associated with the edges or the faces of the two grids. However, we proceed to show that in this case also the field quantities are used in such a way that global quantities are actually intended.

Let us start with the quantity associated with the primary 1-cells $\tau_{1}^{k}$. This quantity is defined by the DSI method to be the projection $\mathbf{E} \cdot \mathbf{s}_{k}$ of the electric field intensity vector $\mathbf{E}$-assumed to be constant along the cell-onto the primary 1 -cells $\tau_{1}^{k}$ represented as a vector $\mathbf{s}_{k}$. This means that a global value is actually considered associated with each primary 1-cell. In fact, if $\mathbf{E}$ is assumed constant also during a time interval $\Delta t$, we can consider directly the global space-time quantity $\phi_{k}^{e}=\mathbf{E} \cdot \mathbf{s}_{k} \Delta t$ thought of as associated with a space-time primary 2-cell since this is the form in which $\mathbf{E}$ always appears in the DSI formulas. Correspondingly, the quantity associated with the secondary 1-cells $\tilde{\tau}_{1}^{k}$ is the projection $\mathbf{H} \cdot \tilde{\mathbf{s}}_{k}$ of the magnetic field intensity vector $\mathbf{H}$-assumed to be constant along the cell-onto the secondary 1-cells $\tilde{\tau}_{1}^{k}$ represented as a vector $\widetilde{\mathbf{s}}_{k}$. This association can also be extended to consider the space-time global quantity $\psi_{k}^{h}=\mathbf{H} \cdot \tilde{\mathbf{s}}_{k} \widetilde{\Delta t}$.

A full magnetic flux density vector $\mathbf{B}$ is associated with each primary 2-cell, and a full electric flux density vector $\mathbf{D}$ is associated with each secondary 2-cell. Both vectors are assumed to be constant over the corresponding cell. Even if these quantities are given as full-vector quantities, they appear in the DSI discretized Maxwell's equations only as $\mathbf{B} \cdot \mathbf{N}_{i}$ and $\mathbf{D} \cdot \tilde{\mathbf{N}}_{i}$, where $\mathbf{N}_{i}$ and $\tilde{\mathbf{N}}_{i}$ are the so-called area-normal vectors, defined so that the two scalar products are actually the magnetic flux $\phi_{i}^{b}=\mathbf{B} \cdot \mathbf{N}_{i}$ and the electric flux $\psi_{i}^{d}=\mathbf{D} \cdot \tilde{\mathbf{N}}_{i}$, associated with the corresponding cells. In this way we have interpreted all DSI variables as global quantities. 
We can now consider the DSI discretization of Faraday's law and MaxwellAmpère's law in light of this interpretation of the variables. Faraday's law at time $t_{n+1 / 2}=(n+1 / 2) \Delta t$ is written for a primary 2-cell $\tau_{2}^{i}$, represented by the vector $\mathbf{N}_{i}$, as

$$
\frac{d \mathbf{B}_{n+1 / 2}}{d t} \cdot \mathbf{N}_{i}=-\int_{\partial \tau_{2}^{i}} \mathbf{E}_{n+1 / 2} \cdot d \mathbf{l}
$$

To discretize the time derivative, the DSI method adopts a time-centered leapfrog algorithm, which sets

$$
\frac{d \mathbf{B}_{n+1 / 2}}{d t}=\frac{\mathbf{B}_{n+1}-\mathbf{B}_{n-1}}{\Delta t}
$$

Substituting Eq. (247) in Eq. (246) we obtain the DSI time-stepping formula for magnetic flux:

$$
\mathbf{B}_{n+1} \cdot \mathbf{N}_{i}=\mathbf{B}_{n} \cdot \mathbf{N}_{i}-\Delta t \int_{\partial \tau_{2}^{i}} \mathbf{E}_{n+1 / 2} \cdot d \mathbf{l}
$$

Remembering the expression of the boundary in terms of incidence numbers, we find that Eq. (248) becomes

$$
\mathbf{B}_{n+1} \cdot \mathbf{N}_{i}=\mathbf{B}_{n} \cdot \mathbf{N}_{i} \pm \sum_{k}\left[\tau_{2}^{i}, \tau_{1}^{k}\right] \mathbf{E}_{n+1 / 2} \cdot \mathbf{s}_{k} \Delta t
$$

(with the uncertainty on the sign of the last term due to the usual dilemma regarding the relative default orientation of primary 1-cells with respect to the default positive direction of $\mathbf{E}$ ). With the aforementioned interpretation of the variables as global quantities, Eq. (249) becomes

$$
\phi_{i, n+1}^{b}=\phi_{i, n}^{b} \pm \sum_{k}\left[\tau_{2}^{i}, \tau_{1}^{k}\right] \phi_{k, n+1 / 2}^{e}
$$

which is exactly the topological time-stepping formula for $\phi^{b}$ of the reference discretization method [Eq. (188)]. The same procedure can be applied to show that the DSI time-stepping formula for $\mathbf{D}$, which is

$$
\mathbf{D}_{n+1 / 2} \cdot \tilde{\mathbf{N}}_{i}=\mathbf{D}_{n-1 / 2} \cdot \tilde{\mathbf{N}}_{i}+\Delta t \int_{\partial \tilde{\tau}_{i}^{i}} \mathbf{H}_{n} \cdot d \mathbf{l}
$$

reduces to the reference topological time-stepping formula for $\psi^{d}$ [Eq. (190)].

It remains now to examine how the DSI method proceeds to the discretization of the constitutive relations. We assume as given the local constitutive equation $\mathbf{B}=\mu \mathbf{H}$, and we consider how it is used to determine a discrete link going from $\phi^{b}$ to $\psi^{h}$. The DSI method adopts a reconstruction-projection method that is a particular case of discretization strategy 2 described previously for the discretization of the constitutive relations. The reconstruction is performed 


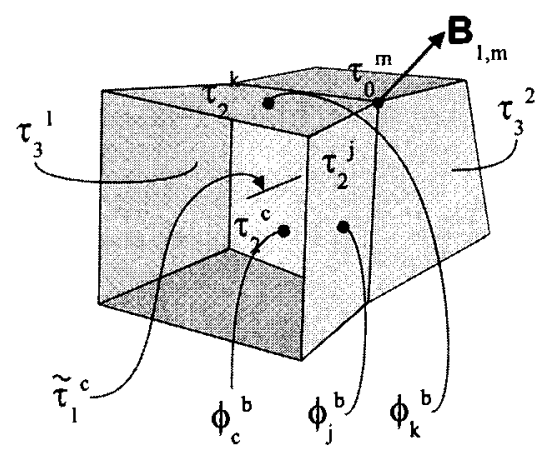

FIGURE 45. The discrete surface integral method adopts a reconstruction-projection strategy for the discretization of the constitutive equations, which is based on the boundary cells of pairs of adjacent 3-cells.

with the following procedure. Consider two adjacent primary 3-cells, $\tau_{3}^{1}$ and $\tau_{3}^{2}$ (Fig. 45). They have in common a primary 2-cell $\tau_{2}^{c}$. The boundary of $\tau_{2}^{c}$ is composed by primary 1-cells whose boundaries constitute a collection of 0 -cells $\tau_{0}^{m}$. The DSI method associates with each of these 0 -cells two magnetic flux density vectors $\mathbf{B}_{1, m}$ and $\mathbf{B}_{2, m}$, one for each of the two 3-cells, $\tau_{3}^{1}$ and $\tau_{3}^{2}$. Each of these vectors is derived from a system of equations asking the fluxes calculated by integrating the vectors on three 2-cells (over which they are assumed as constant) to equal the fluxes associated with these same cells as DSI variables.

In more detail, calling $\tau_{2}^{j}, \tau_{2}^{k}$ the other cells (besides the common cell $\tau_{2}^{c}$ ) belonging to the boundary of $\tau_{3}^{1}$, which meet in the node $\tau_{0}^{m}$; calling $\mathbf{N}_{c}, \mathbf{N}_{j}$, $\mathbf{N}_{k}$ the corresponding area-normal vectors; and calling $\phi_{c}^{b}, \phi_{j}^{b}, \phi_{k}^{b}$ the variables associated with these 2-cells, the DSI method sets

$$
\left\{\begin{array}{l}
\phi_{c}^{b}=\mathbf{B}_{1, m} \cdot \mathbf{N}_{c} \\
\phi_{j}^{b}=\mathbf{B}_{1, m} \cdot \mathbf{N}_{j} \\
\phi_{k}^{b}=\mathbf{B}_{1, m} \cdot \mathbf{N}_{k}
\end{array}\right.
$$

and determines $\mathbf{B}_{1, m}$ in terms of $\phi_{c}^{b}, \phi_{c}^{b}$, and $\phi_{k}^{b}$. The same process is repeated for the adjacent 3 -cell $\tau_{3}^{2}$ to determine $\mathbf{B}_{2, m}$, and for all the nodes $\tau_{0}^{m}$ belonging to the common 2-cell $\tau_{2}^{c}$. The information constituted by all the $\mathbf{B}_{1, m}$ and $\mathbf{B}_{2, m}$ thus determined is then merged by using a weighting formula to produce finally a single vector B. The seminal paper on DSI (Madsen, 1995) examines three weighting formulas for this task.

The vector $\mathbf{B}$ is then assumed to be the reconstructed, constant field within the two adjacent 3-cells, $\tau_{3}^{1}$ and $\tau_{3}^{2}$. It depends on the values of the variable 
$\phi_{c}^{b}$ associated with the common 2-cell $\tau_{2}^{c}$ and on the values $\phi_{i}^{b}$ associated with all the 2-cells belonging to the boundary of $\tau_{3}^{1}$ and $\tau_{3}^{2}$, which touch $\tau_{2}^{c}$. The inverse local constitutive equation $\mathbf{H}=\frac{1}{\mu} \mathbf{B}$ is then applied to the reconstructed field. The resulting field $\mathbf{H}$ must at this point be projected on the secondary 1-cell $\tilde{\tau}_{1}^{c}$, dual to $\tau_{2}^{c}$, for $\mathbf{H} \cdot \tilde{\mathbf{s}}_{c}$ to be determined. This is done by using the formula

$$
\mathbf{H} \cdot \tilde{\mathbf{s}}_{c}=\frac{\mathbf{B} \cdot \tilde{\mathbf{s}}_{c}}{\bar{\mu}_{c}}
$$

where $\bar{\mu}_{c}$ is obtained by averaging $\mu$ along $\tilde{\tau}_{1}^{c}$. Finally the global space-time value $\psi_{c}^{h}=\mathbf{H} \cdot \widetilde{\mathbf{s}}_{c} \widetilde{\Delta t}$ is determined by multiplying the result of Eq. (253) by the time step $\widetilde{\Delta t}$. This whole process produces a discrete constitutive link which relates $\psi_{c}^{h}$ to the values $\phi_{i}^{b}$ on which the reconstructed field $\mathbf{B}$ depends. A similar procedure can be applied to discretize the constitutive equation $\mathbf{D}=\varepsilon \mathbf{E}$, yielding a relation linking the global space-time quantity $\boldsymbol{\phi}_{c}^{e}$ associated with each primary space-time 2-cell to the values $\psi_{k}^{d}$ associated with a small number of secondary neighboring 2-cells. Note that for boundary 2-cells the DSI reconstruction procedure is based on a single 3-cell.

In summary, this analysis shows that the DSI method is, like the FDTD method, fully compliant with the prescriptions of the reference discretization strategy. It adopts a topological time-stepping formula for the global electromagnetic variables, although this remains hidden because of the use of local field quantities in the original description of the method. Compared with the FDTD method, the DSI method defines the quantities involved in more general terms, so that its time-stepping formulas apply to generic unstructured grids (only provided they are two dual cell complexes) on which they preserve their topological nature. Moreover, the DSI method adopts a more sophisticated approach to the discretization of constitutive equations than that of the FDTD method, since the DSI approach is based on a more complex reconstruction-projection strategy. All these properties make the DSI method a generalization of the FDTD method, which, from the point of view of the structure of physical field theories, preserves the favorable characteristics of that method.

Considering in detail from the same point of view its reconstructionprojection strategy, the DSI method appears, however, to be far from optimal. The reconstruction strategy is actually focused on the determination of nodal field quantities and does not make use of edge elements. It is, therefore, likely that experiments with different reconstruction-projection operators more intimately related to the cochain concept would lead to further improvements of the method, not only in terms of compliance with the structure of electromagnetism but also in terms of accuracy. 


\section{The Finite Integration Theory Method}

The finite integration theory (FIT) method is an FV method for time-dependent electromagnetic problems which was developed independently of the FDTD method (Weiland, 1984, 1996). It is interesting to examine this method for two reasons. First, it has undergone a series of improvements from the time of its first appearance in the literature which have made it more and more similar to the reference discretization strategy described previously. Second, it distinguishes well the various phases of the discretization process for a field problem.

In the first phase of its development (Weiland, 1984) the discretization of geometry adopted by the FIT method was based on two dual orthogonal grids, $G$ and $\tilde{G}$. The idea of two kinds of orientation was not explicitly mentioned, but its consequences in terms of association of physical quantities with the two grids were implicitly used. In a more recent reformulation of the FIT method (Weiland, 1996), the orthogonal grids are abandoned and the fact that the two meshes need only be cell complexes is recognized. The new kind of mesh $G$ is constructed by partitioning the spatial domain into volumes $V^{i}$, whose nonempty pairwise intersection is a set of surfaces $A^{j}$, whose pairwise intersection is in turn a set of lines $L^{k}$, whose pairwise intersection is in the end a set of points $P^{l}$. Comparing this procedure with the definition of a cell complex given previously, we recognize in the resulting structure $G$ our primary cell complex $K$, and in the sets $\left\{V^{i}\right\},\left\{A^{j}\right\},\left\{L^{k}\right\}$, and $\left\{P^{l}\right\}$ four sets of $p$-cells $\left\{\tau_{p}^{i}\right\}$, $p=0, \ldots, 3$. The dual mesh $\tilde{G}$, which corresponds to the secondary cell complex $\tilde{K}$, is constructed by defining for each $V^{i}$ of $G$ a dual point $\tilde{P}^{i}$ located within $V^{i}$ and proceeding then to define the other dual objects $\tilde{L}^{j}, \tilde{A}^{k}$, and $\tilde{V}^{l}$.

The discretization of fields, like that of the geometry, changed with the development of the method. Originally (Weiland, 1984) the quantities considered were the field components tangent to the lines of the grid in the case of field intensities $\mathbf{E}$ and $\mathbf{H}$, and the field components perpendicular to the cells in the case of flux densities $\mathbf{B}$ and $\mathbf{D}$. These components were assumed as evaluated at midcell and were subjected to numerical integration to obtain an approximation of the global quantities appearing in Maxwell's equations in integral form. More recent formulations of the FIT method (Weiland, 1996) show that it has been recognized in the meantime that in writing these equations there is no reason to introduce the local field variables first. Consequently, the variables considered became the global field quantities associated with the geometric objects of the meshes. This process, however, was not fully carried out to include space-time geometric objects. Therefore only global quantities associated with spacelike objects are considered. These, if we adapt the notation to that used in the present work, are the electric voltages $V_{k}^{e}$ associated with the primary 1-cells $\tau_{1}^{k} \equiv L^{k}$; the magnetic fluxes $\phi_{c}^{b}$ associated with the primary 2-cells $\tau_{2}^{j} \equiv A^{j}$; the magnetic voltages $F_{j}^{h}$ associated with the secondary 
1-cells $\tilde{\tau}_{1}^{j} \equiv \tilde{L}$; the electric fluxes $\psi_{k}^{d}$ and the electric currents $I_{k}^{j}$ associated with the secondary 2-cells $\tilde{\tau}_{2}^{k} \equiv \tilde{A}^{k}$; and the electric charges $Q_{1}^{\rho}$ associated with the secondary 3 -cells $\tilde{\tau}_{3}^{l} \equiv \tilde{V}^{l}$. In formulas,

$$
\begin{aligned}
V_{k}^{e} & =\int_{\tau_{1}^{k}} \mathbf{E} \\
\phi_{j}^{b} & =\int_{\tau_{2}^{\prime}} \mathbf{B} \\
F_{j}^{h} & =\int_{\tilde{\tau}_{1}^{\prime}} \mathbf{H} \\
\psi_{k}^{d} & =\int_{\tilde{\tau}_{2}^{k}} \mathbf{D} \\
I_{k}^{j} & =\int_{\tilde{\tau}_{2}^{k}} \mathbf{J} \\
Q_{l}^{\rho} & =\int_{\tilde{\tau}_{3}^{\prime}} \rho
\end{aligned}
$$

Note that if we consider only the geometric objects in space, two distinct quantities of the same physical theory appear as associated with the same geometric object $\tilde{\tau}_{2}^{k}$. The variables just defined are grouped by the FIT method into vectors $\mathbf{V}^{e}, \Phi^{b}, \tilde{\mathbf{F}}^{h}, \tilde{\Psi}^{d}, \tilde{\mathbf{I}}^{j}$, and $\mathbf{Q}^{\rho}$, which we can interpret as natural representations of space-like cochains.

The discretization of topological laws at this point follows easily. Maxwell's equations in integral form with respect to space, but in differential form with respect to time, are considered [Eqs. (23), (28), (29), and (24)]. Using implicitly the coboundary operator in space, these equations are semi-discretized, that is, the spacelike part of the topological relation is written in terms of the preceding cochains, and the time derivative is left in its original differential form. In matricial form, this reads

$$
\begin{aligned}
\frac{d \Phi^{b}}{d t}+\mathbf{D}_{2,1} \mathbf{V}^{\mathbf{e}} & =0 \\
\mathbf{D}_{3,2} \Phi^{b} & =0 \\
-\frac{d \tilde{\Psi}^{d}}{d t}+\tilde{\mathbf{D}}_{2,1} \tilde{\mathbf{F}}^{h} & =\tilde{\mathbf{I}}^{j} \\
\tilde{\mathbf{D}}_{3,2} \tilde{\Psi}^{d} & =\tilde{\mathbf{Q}}^{\rho}
\end{aligned}
$$

where $\mathbf{D}_{2,1}$ and $\mathbf{D}_{3,2}$ are the incidence matrices on the primary cell complex, and $\tilde{\mathbf{D}}_{2,1}$ and $\tilde{\mathbf{D}}_{3,2}$ those on the secondary complex. The following relations 
hold true (Weiland, 1996):

$$
\begin{aligned}
\mathbf{D}_{2,1} & =\tilde{\mathbf{D}}_{2,1}^{T} \\
\mathbf{D}_{3,2} \mathbf{D}_{2,1} & =0 \\
\tilde{\mathbf{D}}_{3,2} \tilde{\mathbf{D}}_{2,1} & =0
\end{aligned}
$$

Since the incidence matrices are the matricial representations of the coboundary operator, these relations correspond to the aforementioned adjointness of pairs of coboundary operators acting on the primary and secondary meshes, and to the relation $\delta \delta=0$ considered on the primary and secondary meshes.

The constitutive equations are then discretized. The literature on the method is somewhat vague about the details of this process. The method used seems to correspond to discretization strategy 1 (discussed previously); that is, two dual cells are considered and the local constitutive equation is extended to the global quantities associated with these cells. For example, to discretize the constitutive equation $\mathbf{B}=\mu \mathbf{H}$, two dual orthogonal cells, $\tau_{2}^{j}$ and $\tilde{\tau}_{1}^{j}$, are considered, and the corresponding global quantities are assumed as linked by a relation of the kind

$$
\frac{\phi_{j}^{b}}{F_{j}^{h}}=C_{\mu, j}
$$

The coefficients $C_{\mu, j}$ constitute a matrix $C_{\mu}$, which is diagonal for simple constitutive equations and meshes having orthogonal dual cells but can be nondiagonal in more complex cases. The discrete constitutive equations are, therefore, linear relations of the kind

$$
\begin{aligned}
\tilde{\Psi}^{d} & =\mathbf{C}_{\varepsilon} \mathbf{V}^{e} \\
\Phi^{b} & =\mathbf{C}_{\mu} \tilde{\mathbf{F}}^{h} \\
\tilde{\mathbf{I}}_{L}^{j} & =\mathbf{C}_{\sigma} \mathbf{V}^{e}
\end{aligned}
$$

where the subscript in the term $\tilde{\mathbf{I}}_{L}^{j}$ signals that there can be other contributions to the electric current besides this one. Using these equations, we find that the semidiscretized Maxwell's equations [Eqs. (260) through (263)] can be rewritten in terms of two cochains only, which the FIT method chooses to be $\Phi^{b}$ and $\mathbf{V}^{e}$. In particular, besides Eq. (260), which already depends on these two quantities only, the time-dependent relation [Eq. (262)], which corresponds to Maxwell-Ampère's law, becomes

$$
-\frac{d}{d t}\left(\mathbf{C}_{\varepsilon} \mathbf{V}^{e}\right)+\tilde{\mathbf{D}}_{2,1} \mathbf{C}_{\mu}^{-1} \Phi^{b}=\tilde{\mathbf{I}}^{\prime}
$$

The set of semidiscrete equations obtained in this way are called Maxwell grid equations. 
Note that to this point no time-stepping procedure has been defined. From the choice of $\Phi^{b}$ and $\mathbf{V}^{e}$ as privileged variables, it follows that the time-stepping process will be based on the two time-dependent semidiscretized equations in terms of $\Phi^{b}$ and $\mathbf{V}^{e}$ [Eqs. (260) and (271)]. Various strategies for the discretization of the time derivatives remaining in these two equations are considered. In particular the usual leapfrog method is pointed out as the method of choice in most cases. For a time step $\Delta t$ this gives the following time-stepping formulas (Weiland, 1996):

$$
\begin{aligned}
\Phi_{n+1}^{b} & =\Phi_{n}^{b}-\Delta t \mathbf{D}_{2,1} \mathbf{V}_{n+1 / 2}^{\mathbf{e}} \\
\mathbf{V}_{n+1 / 2}^{e} & =\mathbf{V}_{n-1 / 2}^{e}+\Delta t \mathbf{C}_{\varepsilon}^{-1}\left(\tilde{\mathbf{D}}_{2,1} \mathbf{C}_{\mu}^{-1} \Phi_{n}^{b}-\tilde{\mathbf{I}}_{n}^{j}\right)
\end{aligned}
$$

These formulas cannot be considered topological time-stepping relations. To arrive at a topological time-stepping relation, we must first rearrange them as follows:

$$
\begin{aligned}
\Phi_{n+1}^{b} & =\Phi_{n}^{b}-\mathbf{D}_{2,1} \Delta t \mathbf{V}_{n+1 / 2}^{\mathbf{e}} \\
\mathbf{C}_{\varepsilon} \mathbf{V}_{n+1 / 2}^{e} & =\mathbf{C}_{\varepsilon} \mathbf{V}_{n-1 / 2}^{e}+\left(\tilde{\mathbf{D}}_{2,1} \Delta t \mathbf{C}_{\mu}^{-1} \Phi_{n}^{b}-\Delta t \tilde{\mathbf{I}}_{n}^{j}\right)
\end{aligned}
$$

and then, considering the constitutive relations [Eqs. (268) and (269)]; introducing the cochains $\tilde{\Psi}^{h}, \Phi^{e}$, and $\tilde{\mathbf{Q}}^{j}$; and putting $\Delta t \tilde{\mathbf{F}}^{h}=\tilde{\Psi}^{h}, \Delta t \mathbf{V}^{e}=\Phi^{e}$, and $\Delta t \tilde{\mathbf{I}}^{j}=\tilde{\mathbf{Q}}^{j}$, we can finally rewrite them as topological time-stepping relations

$$
\begin{aligned}
\Phi_{n+1}^{b} & =\Phi_{n}^{b}-\mathbf{D}_{2,1} \Phi_{n+1 / 2}^{\mathrm{e}} \\
\tilde{\Psi}_{n+1 / 2}^{d} & =\tilde{\Psi}_{n-1 / 2}^{d}+\left(\tilde{\mathbf{D}}_{2,1} \tilde{\Psi}_{n}^{h}-\tilde{\mathbf{Q}}_{n}^{j}\right)
\end{aligned}
$$

which correspond to those of the reference discretization strategy.

In summary, the developers of the FIT method appear to have recognized, in the course of its evolution, the desirability of adopting a number of features that are suggested by the structural analysis of physical theories. These include the choice of cell complexes to discretize the domain and the priority of global physical quantities associated with geometric objects over local field quantities and their adoption as the method's variables. Moreover, the distinction of topological laws from constitutive relations was built into the method from the start, along with the preservation in the semidiscrete system of equations of many structural properties of the continuous model of the original problem.

The strategy adopted for the discretization of constitutive equations appears, however, elementary. Moreover, the method falls short of recognizing the desirability of a truly space-time approach to the discretization. The resulting choice of variables in the time-stepping formulas and the time-stepping formulas themselves suffer from this oversight. Even with the adoption of leapfrog time stepping, the interpretation of the FIT time-stepping formula 
as a topological time stepping appears artificial, while the properties of the continuous mathematical model that were preserved in the semidiscrete model are at risk of being lost in the time discretization step.

\section{Finite Element Methods}

Originally, the finite element (FE) method was conceived of as an analytical tool for solid mechanics, and its first formulation was based on a direct physical approach (Burnett, 1987; Fletcher, 1984). Given its flexibility with respect to FD methods and the good results produced, the FE approach was applied to many other fields, with the variations required by the nature of the new problems. A whole class of FE methods ensued, which were soon given a rigorous mathematical foundation using the ideas of functional analysis. Despite this later formalization, the origins of the method lead one to expect that a certain similarity exists between the FE approach to discretization and the "physical" one of the reference discretization strategy. Let us, therefore, examine the FE methods from this point of view.

We must first define what we intend to consider as an FE method, and we will do this in operative terms. To speak in concrete terms, let us consider a simple electrostatic problem. We assume that a distribution of charge $\rho$ is given in a domain $D$, along with suitable boundary conditions along $\partial D$, and we seek the electrostatic potential $V$ on $D$. We know (Fig. 18) that the field equations for this problem can be factorized into the following pair of topological equations

$$
\begin{aligned}
-\operatorname{grad} V & =\mathbf{E} \\
\operatorname{div} \mathbf{D} & =\rho
\end{aligned}
$$

supplemented by a constitutive equation of the kind

$$
\mathbf{D}=f_{\varepsilon}(\mathbf{E})
$$

The FE discretization procedure starts with the subdivision of the $n$ dimensional domain $D$ in elements. In the simplest cases the elements correspond to the $n$-cells of the reference method and define a mesh in the domain. The field quantities that have been selected as unknowns are then given a discrete representation. This is done in terms of a finite number of variables associated with geometric objects, which belong to the mesh. In our case, since the unknown is a 0 -field, these objects are a set of 0 -cells, the so-called nodes in FE terminology. Two possibilities are open at this point for the discretization of the equations: the variational approach and the weighted residual approach (Fletcher, 1984). Given the greater generality of the latter, we will consider the weighted residual approach as characteristic of FE methods. To apply this technique, we must consider the complete field equation; that is, we must 
reassemble Eqs. (278) through (280) to give

$$
-\operatorname{div}\left(f_{\varepsilon}(\operatorname{grad} V)\right)=\rho
$$

The first step of the FE discretization of this continuous formulation of the problem consists of a reconstruction of the unknown quantities based on its discrete representation, using a set of shape functions $s_{j}(\mathbf{r})$ (see the section on edge elements-Section IV.A.4), as follows:

$$
V(\mathbf{r})=\sum_{j} V_{j} s_{j}(\mathbf{r})
$$

This transforms Eq. (281) into

$$
\operatorname{div}\left(f_{\varepsilon}\left(\operatorname{grad}\left(\sum V_{j} s_{j}\right)\right)\right)=\rho
$$

Despite the adoption of a discrete representation for the unknown field, Eq. (283) is still a partial differential equation. So that a system of algebraic equations can be obtained from it, a set of weight functions $w^{i}$ is selected, and the following set of residual equations is written:

$$
-\int_{D} \operatorname{div}\left(f_{\varepsilon}\left(\operatorname{grad}\left(\sum V_{j} s_{j}\right)\right)\right) w^{i}=\int_{D} \rho w^{i} \quad \forall i
$$

To obtain a sparse coefficient matrix, FE methods adopt shape and weight functions having local character. Therefore, the support of each weight function is a small subdomain of $D$, which is in fact a 3-cell that we can denote with $\tau_{3}^{i}$. Using the prior notation for weighted integrals, we can rewrite Eq. (284) as follows:

$$
-\int_{w^{i} \tau_{3}^{i}} \operatorname{div}\left(f_{\varepsilon}\left(\operatorname{grad}\left(\sum V_{j} s_{j}\right)\right)\right)=\int_{w^{i} \tau_{3}^{i}} \rho \quad \forall i
$$

The left side of each of these equations can be integrated by parts, which gives

$$
-\int_{\partial\left(w^{i} \tau_{3}^{i}\right)} f_{\varepsilon}\left(\operatorname{grad}\left(\sum V_{j} s_{j}\right)\right)=\int_{w^{i} \tau_{3}^{i}} \rho \quad \forall i
$$

where the meaning of the term on the left side is defined by Eq. (172). The set of equations represented by Eq. (286) is the system of algebraic equations produced by the FE method.

We can interpret this strategy in light of the reference method. First, we must reconstruct the field $V$, starting from the 0-cochain $\mathbf{V}^{v}=\left\{V_{j}\right\}$, using the shape functions $s_{j}$. We can represent this as the action of a reconstruction operator $R^{v}$ as follows:

$$
V=R^{v}\left(\mathbf{V}^{v}\right)
$$


Next, we apply the local topological relation [Eq. (278)] to the reconstructed field, which gives the $\mathbf{E}$ field. The constitutive relation [Eq. (280)] is then applied to $\mathbf{E}$, and we obtain the electric flux density $\mathbf{D}$. For each weight function, that is, on each spread cell $w_{j} \tau_{3}^{i}$, we finally impose, the following topological equation:

$$
\int_{\partial\left(w^{i} \tau_{3}^{i}\right)} \mathbf{D}=\int_{w^{i} \tau_{3}^{i}} \rho \quad \forall i
$$

Compared with the reference strategy, which enforces both topological equations in discrete terms on crisp cells, the approach just described differs in its applying one topological equation in differential terms and the other in integral terms on spread cells. The difference could be reduced by reformulating the reconstruction of $\mathbf{E}$ and making it start from the $\mathbf{V}^{e}$ cochain, and then applying to it the corresponding topological equation in coboundary terms. In any case the projection of the reconstructed field is not performed, because of the presence of (secondary) spread cells, which, contrary to the case of crisp cells, do not require this step. Note that if the reconstruction is based on $\mathbf{V}^{e}$, edge elements and not nodal interpolation must be used to obtain a physically sound reconstruction of $\mathbf{E}$. This is always true in the case of magnetostatics problems, since the magnetic potential $\mathbf{A}$ is a 1-field and a correct reconstruction of it must start from the cochain $V^{a}$ and use (ordinary) 1-edge elements. The realization of this fact was one of the reasons that led to the introduction of edge elements by the computational electromagnetics community.

This analysis reveals also the different role of shape functions and weight functions in the discretization process. Shape functions are used to reconstruct the fields in order to approximate the constitutive equations, whereas weight functions define the spread cells which constitute a continuous counterpart of the secondary mesh to which the corresponding topological equations are applied. With different joint choices of the two sets of functions we obtain different categories of methods. If the weight functions $w^{i}$ are the characteristic functions of their support $\tau_{3}^{i}$, that is, if

$$
w^{i}= \begin{cases}1 & \text { in } \tau_{3}^{i} \\ 0 & \text { outside } \tau_{3}^{i}\end{cases}
$$

then the method is called a subdomain method. Considering Eq. (287), we recognize that a subdomain method is actually an FV method, since it applies the topological equations to a set of crisp cells $\tau_{3}^{i}$. If the weight functions coincide with the shape functions, that is, if

$$
w^{i}(\mathbf{r})=s_{i}(\mathbf{r}) \quad \forall i
$$


then the method is called a Galerkin method. Other choices are, of course, possible and give rise, for example, to the so-called collocation method and to the least squares method (Fletcher, 1984).

The choice corresponding to the Galerkin method is undoubtedly the most used in FE. This is linked to the fact that, when a variational formulation exists for the problem, Galerkin's choice gives a system of equations that corresponds to that derived from the variational approach. Considering their different roles in the discretization process, the systematic choice of coincident weight and shape functions appears, however, to be questionable. If edge elements are adopted as shape functions on the grounds of physical considerations linked to the association of physical quantities with geometric objects, then, in the same spirit, weight functions should be chosen in order to impose in an optimal way the topological equations, and it may turn out that some kinds of shape functions are not ideally suited to this task. [For an analysis of the different roles of shape and weight functions from a functional analysis viewpoint, see Schroeder and Wolff (1994).]

\section{Time-Domain Finite Element Methods}

The FE discretization strategy exemplified in the preceding paragraphs does not lend itself easily to the discretization of time-dependent problems. It has been noted (Fletcher, 1984) that the classical FE method is intrinsically "elliptic," in the sense that it solves problems by "propagating" simultaneously on the whole domain the source and boundary conditions of the problem. Therefore it is ideally suited to the solution of boundary-value problems but does not apply well to initial-value problems. To adapt the nature of the FE method to a transient problem defined in a time interval $\left[t_{0}, t_{1}\right]$, one should consider space-time shape functions $\mathbf{s}(\mathbf{r}, t)$ and weight functions $\mathbf{w}(\mathbf{r}, t)$, and transform the initial-boundary-value problem in a boundary-value problem, generating in some way the missing boundary condition at the final time instant $t=t_{1}$. This can be done, for example, by putting $t_{1}=\infty$ and using the steady-state solution of the problem with time-infinite elements, or by using finite elements and a $t_{1}$ large enough to make the solution at that time sufficiently similar to the steady-state condition (Burnett, 1987). Understandably, neither of these approaches enjoyed great popularity.

A first alternative to this approach is the adoption of the separation of variables technique. Assume that, as usual, the problem constituted by Faraday's law [Eq. (8)] and Maxwell-Ampère's law [Eq. (13)], with the simple constitutive equations of electromagnetics [Eqs. (20) through (22)], and with suitable initial and boundary conditions, is to be considered. As for the preceding electrostatic problem, we first combine the equations into a single partial 
differential equation, for example (Lee et al., 1997)

$$
\operatorname{curl}\left(\frac{1}{\mu} \operatorname{curl} \mathbf{E}\right)+\varepsilon \frac{\partial^{2} \mathbf{E}}{\partial t^{2}}+\sigma \frac{\partial \mathbf{E}}{\partial t}+\frac{\partial \mathbf{J}_{i}}{\partial t}=0
$$

where $\mathbf{J}_{i}$ are the impressed currents. The space-time shape functions are expressed as products of functions that depend separately on space and time, and the unknown field is reconstructed as follows:

$$
\mathbf{E}(\mathbf{r}, t)=\sum V_{j}^{e}(t) \mathbf{s}_{j}(\mathbf{r})
$$

In this case, the shape functions are assumed to be 1-edge elements, and the vector of coefficients $\left\{V_{j}^{e}(t)\right\}$ can be considered a time-dependent cochain $\mathbf{V}^{e}(t)$. Next, a set of suitable weight functions $\mathbf{w}^{i}(\mathbf{r})$ is considered at a generic time instant $t$, and the weighted residual method is applied to Eq. (290), which results in a system of ordinary differential equations

$$
\mathbf{A} \frac{d^{2} \mathbf{V}^{e}}{d t^{2}}+\mathbf{B} \frac{d \mathbf{V}^{e}}{d t}+\mathbf{C} \mathbf{V}^{e}+\mathbf{d}=0
$$

where $\mathbf{A}, \mathbf{B}$, and $\mathbf{C}$ are matrices and $\mathbf{d}$ is a vector. This system of equations is finally discretized and solved by using some time-stepping method for ordinary differential equations.

The approach just examined, because of its adoption of edge elements for the reconstruction, has in common with the approach of the reference discretization strategy the discrete representation of a field as a cochain. However, the similarities stop there, since the discrete representation of fields does not extend to space-time and the distinction of topological and constitutive equations is not recognized. Moreover, contrary to the case of the electrostatic problem, in which the distinction was not explicitly recognized by the FE approach but could be considered implicitly built into the method, disentanglement is not possible here since Eq. (290) mixes inextricably the two time-dependent Maxwell's equations and the constitutive equations. The same holds true for the other approach to time-dependent problems that preserves to the problem the "ellipticity" suited to the classical FE approach, that which considers time-harmonic fields (Jin, 1993). In that case Eq. (290) becomes

$$
\operatorname{curl}\left(\frac{1}{\mu} \operatorname{curl} \mathbf{E}\right)-\omega^{2} \varepsilon \mathbf{E}+j \sigma \omega \mathbf{E}+j \omega \mathbf{J}_{i}=0
$$

where not only are the equations mixed, but the possibility of a space-time approach is also definitely lost.

Thus it seems that despite its physical origin, the classical FE approach is not capable of producing a truly physical discretization of time-dependent problems. This is even more surprising if one considers that we owe to the FE method ideas such as that of edge elements, of the reconstruction-projection 
strategy, and of the error-based strategies for constitutive equations discretization. FE practitioners are aware of the problem constituted by the lack of a convincing FE treatment of transient problems, and in recent times have considered with interest the simplicity and effectiveness of the FDTD method (Lee et al., 1997). They have realized in particular that to obtain a physical discretization, one has to start from the factorized equations, that is, consider separately the two time-dependent Maxwell's equations, and the constitutive equations. Let us examine two methods which adopt this discretization philosophy.

\section{Time-Domain Edge Element Method}

An FE-like time-domain method directly based on the two time-dependent Maxwell's equations has been suggested, with minor variations, by many authors. This method is described in the survey paper on time-domain FE methods by Lee et al. (1997). It adopts a single discretization mesh for the domain in space and defines as variables the global quantities associated with the $p$-cells of this mesh. Contrary to the reference method, therefore, we do not have two distinct dual meshes. However, since both the primary and the secondary variables are associated with the cells of the unique mesh, we can assume that two "logical" meshes, which have different kinds of orientations and share the same geometric support, are implicitly defined. In other words, we have $\tau_{n}^{i}=\tilde{\tau}_{n}^{i}$, for every $i$ and for $n=0,1,2,3$. With this provision the variables of the method are defined like those of the FIT method, by Eqs. (254) through (258), and are the electric voltages $V_{k}^{e}$, the magnetic fluxes $\phi_{i}^{b}$, the magnetic voltages $F_{h}^{k}$, the electric fluxes $\psi_{i}^{d}$, and the electric currents $l_{i}^{j}$. Therefore, the fields are correctly represented by cochains, which we denote as $\mathbf{V}^{e}, \Phi^{b}$, $\tilde{\mathbf{F}}^{h}, \tilde{\Psi}^{d}$, and $\tilde{\mathbf{I}}^{j}$, like those of the FIT method.

According to the FE tradition exemplified in the preceding electrostatic example, the method then proceeds to the reconstruction of the fields $\mathbf{E}, \mathbf{B}, \mathbf{H}$, $\mathbf{D}$, and $\mathbf{J}$, using as shape functions a set of 1-edge elements $\mathbf{s}_{k}^{1}$, and a set of 2-edge elements $\mathbf{s}_{i}^{2}$, as in

$$
\begin{aligned}
\mathbf{E} & =\sum_{k} V_{k}^{e} \mathbf{s}_{k}^{1}=R_{e}\left(\mathbf{V}^{e}\right) \\
\mathbf{H} & =\sum_{k} F_{k}^{h} \mathbf{s}_{k}^{1}=R_{h}\left(\tilde{\mathbf{F}}^{h}\right) \\
\mathbf{D} & =\sum_{i} \psi_{i}^{d} \mathbf{s}_{i}^{2}=R_{d}\left(\tilde{\Psi}^{d}\right) \\
\mathbf{B} & =\sum_{i} \phi_{i}^{b} \mathbf{s}_{i}^{2}=R_{b}\left(\Phi^{b}\right) \\
\mathbf{J} & =\sum_{i} I_{i}^{j} \mathbf{s}_{i}^{2}=R_{j}\left(\tilde{\mathbf{I}}^{j}\right)
\end{aligned}
$$

These expressions are substituted into Maxwell's time-dependent equations and then the collocation method is applied to the resulting equations. This means that Maxwell's equations are applied in integral form to the 2-cells. 
After application of Stokes's theorem, this produces

$$
\begin{aligned}
& \int_{\partial \tau_{2}^{i}} \sum_{k} V_{k}^{e} \mathbf{s}_{k}^{1}+\frac{d}{d t} \int_{\tau_{2}^{i}} \sum_{i} \phi_{i}^{b} \mathbf{s}_{i}^{2}=0 \\
& \int_{\partial \tau_{2}^{i}} \sum_{k} F_{k}^{h} \mathbf{s}_{k}^{1}-\frac{d}{d t} \int_{\tau_{2}^{i}} \sum_{i} \psi_{i}^{d} \mathbf{s}_{i}^{2}=\int_{\tau_{2}^{i}} \sum I_{i}^{j} \mathbf{s}_{i}^{2}
\end{aligned}
$$

which corresponds to

$$
\begin{aligned}
& \sum_{k} V_{k}^{e} \int_{\partial \tau_{2}^{i}} \mathbf{s}_{k}^{1}+\frac{d}{d t} \sum_{i} \phi_{i}^{b} \int_{\tau_{2}^{i}} \mathbf{s}_{i}^{2}=0 \\
& \sum_{k} F_{k}^{h} \int_{\partial \tau_{2}^{\prime}} \mathbf{s}_{k}^{1}-\frac{d}{d t} \sum_{i} \psi_{i}^{d} \int_{\tau_{2}^{i}} \mathbf{s}_{i}^{2}=\sum_{i} I_{i}^{j} \int_{\tau_{2}^{i}} \mathbf{s}_{i}^{2}
\end{aligned}
$$

Remembering the characteristic property of edge elements expressed by Eq. (219), and expressing the boundaries of cells in terms of incidence numbers, we find that this corresponds to

$$
\begin{aligned}
\frac{d \Phi^{b}}{d t}+\mathbf{D}_{2,1} \mathbf{V}^{\mathbf{e}} & =0 \\
-\frac{d \tilde{\Psi}^{d}}{d t}+\mathbf{D}_{2,1} \tilde{\mathbf{F}}^{h} & =\tilde{\mathbf{I}}^{j}
\end{aligned}
$$

where $\mathbf{D}_{2,1}$ is the incidence matrix between 2-cells and 1-cells of the mesh.

Note that this corresponds to the semidiscrete relations [Eqs. (260) and (262)] of the FIT method. This is not a surprise, since we are actually performing the same steps of the FIT method. The fact that a reconstruction of the field quantities is performed before the enforcement of Maxwell's equations is a heritage of the FE approach, but appears completely superfluous, since the subsequent projection performed while the equations are being enforced in integral form reproduces exactly the starting cochain. This is in fact the characteristic property of edge elements, as expressed by Eq. (219), or Eq. (210). The reconstruction is actually required only in a later phase, that is, when it is time to determine the discrete constitutive equations, expressing $\mathbf{V}^{e}$ in terms of $\tilde{\Psi}^{d}$, and $\tilde{\mathbf{F}}^{h}$ in terms of $\Phi^{b}$. This is done by imposing on the reconstructed fields [Eq. (294)] the constitutive equations, and then projecting the result to obtain a cochain, according to

$$
\begin{aligned}
V_{k}^{e} & =\int_{\tau_{1}^{k}} \frac{1}{\varepsilon} \mathbf{D}=P^{e}\left(f_{\varepsilon^{-1}}\left(R_{d}\left(\tilde{\Psi}^{d}\right)\right)\right) \\
F_{k}^{h} & =\int_{\tau_{1}^{k}} \frac{1}{\mu} \mathbf{B}=P^{h}\left(f_{\mu^{-l}}\left(R_{b}\left(\Phi^{b}\right)\right)\right)
\end{aligned}
$$


This, after substitution and integration, gives the matricial links

$$
\begin{aligned}
\mathbf{V}^{e} & =\mathbf{C}_{\varepsilon^{-1}} \tilde{\Psi}^{d} \\
\tilde{\mathbf{F}}^{h} & =\mathbf{C}_{\mu^{-1}} \Phi^{b}
\end{aligned}
$$

Substituting these links in the semidiscrete relations [Eqs. (299) and (300)], and discretizing in time by using a leapfrog scheme, we have

$$
\begin{aligned}
\Phi_{n+1}^{b} & =\Phi_{n}^{b}-\mathbf{D}_{2,1} \Delta t \mathbf{C}_{\varepsilon^{-1}} \tilde{\Psi}^{d} \\
\tilde{\Psi}_{n+1 / 2}^{d} & =\tilde{\Psi}_{n-1 / 2}^{d}+\mathbf{D}_{2,1} \Delta t \mathbf{C}_{\mu^{-1}} \Phi^{b}-\Delta t \tilde{\mathbf{I}}^{j}
\end{aligned}
$$

and finally, putting $\mathbf{F}_{\varepsilon^{-1}}=\Delta t \mathbf{C}_{\varepsilon^{-1}}, \mathbf{F}_{\mu^{-1}}=\Delta t \mathbf{C}_{\mu^{-1}}$, and $\tilde{\mathbf{Q}}^{j}=\Delta t \tilde{\mathbf{I}}^{j}$ gives

$$
\begin{aligned}
\Phi_{n+1}^{b} & =\Phi_{n}^{b}-\mathbf{D}_{2,1} \mathbf{F}_{\varepsilon^{-1}} \tilde{\Psi}^{d} \\
\tilde{\Psi}_{n+1 / 2}^{d} & =\tilde{\Psi}_{n-1 / 2}^{d}+\mathbf{D}_{2.1} \mathbf{F}_{\mu^{-1}} \Phi^{b}-\tilde{\mathbf{Q}}^{j}
\end{aligned}
$$

These time-stepping formulas coincide with those of the reference method [Eqs. (192) and (193)]. In particular they could be put in the from of Eqs. (211) and (212), since the discrete constitutive links are determined by using a reconstruction-projection strategy.

In summary, the time-domain edge element method just described appears to be in fact a particular FV method, which adopts coincident primary and secondary meshes, applies the topological equations in terms of cochains (the premature reconstruction of fields prior to topological equations application being immaterial), and discretizes the constitutive equations by using a reconstruction-projection method based on edge elements. Contrary to the reference discretization strategy, in this method the global variables are not considered associated with space-time geometric objects. However, thanks to its applying the topological equations to crisp cells, its time-stepping formulas can be considered as implementing a topological time stepping.

\section{Time-Domain Error-Based FE Method}

The examples of FE methods given so far and the accompanying discussion could have created in the reader the impression that it is not possible to build a truly "physical" time-domain method by using an FE approach based on spread cells. To prevent the formation of this premature conclusion we present now an example of an interesting error-based time-domain FE method which uses spread cells (Albanese et al., 1994; Albanese and Rubinacci, 1998).

The method is based on the use of potentials both on the primary mesh and on the secondary mesh. To this end, a slightly modified form of Maxwell's 
equations must be considered; that is, the set

$$
\begin{array}{r}
\operatorname{curl} \mathbf{E}+\frac{\partial \mathbf{B}}{\partial t}=\mathbf{0} \\
\operatorname{curl} \mathbf{H}-\frac{\partial \mathbf{D}_{\mathrm{T}}}{\partial t}=\mathbf{0}
\end{array}
$$

where $\mathbf{D}_{\mathrm{T}}$ is a modified electric flux density, which includes the current density term. In this way both these statements appear as flux conservation statements, and the corresponding quantities admit two potentials $\mathbf{A}$ and $\mathbf{W}$, such that (Albanese et al., 1994)

$$
\begin{aligned}
\mathbf{E} & =-\frac{\partial \mathbf{A}}{\partial t} \\
\mathbf{H} & =\frac{\partial \mathbf{W}}{\partial t} \\
\mathbf{B} & =\mathbf{B}_{0}+\operatorname{curl} \mathbf{A} \\
\mathbf{D}_{\mathrm{T}} & =\mathbf{D}_{\mathrm{T}_{0}}+\operatorname{curl} \mathbf{W}
\end{aligned}
$$

The potentials $\mathbf{A}(\mathbf{r}, t)$ and $\mathbf{W}(\mathbf{r}, t)$ are formally reconstructed by using edge elements, as follows:

$$
\begin{aligned}
\mathbf{A} & =R_{a}\left(\mathcal{U}^{a}\right) \\
\mathbf{W} & =R_{w}\left(\Gamma^{w}\right)
\end{aligned}
$$

where $\mathcal{U}^{a}$ and $\Gamma^{w}$ are the corresponding cochains. Next, Eqs. (310) through (313) are used to determine the fields. This ensures that the fields satisfy Eqs. (308) and (309). As a way to enforce the constitutive equations, an error density function $\xi\left(\mathbf{E}, \mathbf{D}_{\mathrm{T}}, \mathbf{B}, \mathbf{H}\right)$ is defined, following the criteria sketched in the definition of Eq. (215), and is integrated in space over the domain $D$, and in time over a time step $\Delta t$, which gives an error functional

$$
\mathcal{E}=\int_{t_{n}}^{t_{n+1}} \int_{D} \xi\left(\mathbf{E}, \mathbf{D}_{\mathrm{T}}, \mathbf{B}, \mathbf{H}\right)
$$

Given Eqs. (314) and (315), the error functional $\mathcal{E}$ can be expressed in terms of the cochains $\mathcal{U}^{a}$ and $\Gamma^{w}$. Therefore, a minimization problem based on $\mathcal{E}$ can be established at each time step, as follows,

$$
\left\{\underline{\mathcal{U}}_{n+1}^{a}, \Gamma_{n+1}^{w}\right\}=\left\{\mathcal{U}_{n+1}^{a}, \Gamma_{n+1}^{w}\right\}: \min _{\left\{\mathcal{U}_{n+1}^{a}, \Gamma_{n+1}^{w}\right.} \mathcal{E}\left(\left\{\mathcal{U}_{n+1}^{a}, \Gamma_{n+1}^{w}\right\},\left\{\mathcal{U}_{n}^{a}, \Gamma_{n}^{w}\right\}\right)
$$

which thus allows the time stepping of the potential cochains. 
From the physical point of view this approach to the solution of Maxwell's equations leaves much to be desired. In particular this is true for its use of a modified form of Maxwell's equations. However, it appears to be a promising idea toward the determination of a physically consistent method based on the traditional approach of FE methods, and for the extension of the error-based methods to the case of time-dependent problems, by adopting either the FE or the FV approach.

\section{Conclusions}

We have presented a set of conceptual tools for the formulation of physical field problems in discrete terms. These tools allow the representation of the geometry and of the fields in discrete terms, by using the concepts of oriented cell complex and chain and cochain. Moreover they allow us to bridge the gap between the continuous and the discrete concepts of field by means of the idea of a limit system. The analysis of the structure of physical field theories is based on these tools. This analysis unveils the importance of thinking of physical quantities as associated with space-time oriented geometric objects. It shows also that these objects must be thought of as endowed with one of two kinds of orientation. Moreover, this analysis exposes the distinction of topological laws from constitutive relations, showing their different behavior from the point of view of their discretizability. It clarifies also that a privileged discrete operator-the coboundary operator-exists for the representation of topological laws.

A reference discretization strategy, which complies with these concepts, has been presented. It is based on the idea of topological time stepping for timedependent equations, which operates on global quantities and derives from the application of the coboundary operator in space-time. It was then shown how topological time stepping can be combined with different strategies for the discretization of the constitutive relations. In particular, three of these strategies were presented and examined in detail.

Analyzing the operation of a number of popular methods, we have shown that there has been a steady tendency of numerical methods devoted to field problems toward the adoption and inclusion of techniques that adhere to the philosophy described previously. In particular we have revealed that many methods can be thought of as implicitly adopting the topological time stepping procedure. According to the signaled trend, even if the concept of topological time stepping seems to have eluded the creators of these methods so far, we can expect it to be recognized and included explicitly in future formulations of these methods. 
In the long run, this trend will probably lead to classes of methods modeled on the reference discretization strategy, which mix the best features of the various methods. In particular, we have shown that methods such as the finite difference and the finite volume methods, which do well with regard to time stepping, usually fail to give the constitutive relations an adequate treatment, thus ending with very crude discretizations that are scarcely applicable to nonstructured grids. On the contrary, finite element methods, which discretize well the constitutive relations and can deal easily with arbitrary meshes, fail with regard to topological laws, especially when such methods are applied to timedependent problems. Thus, the time seems ripe for the combination of the best features of these categories of methods, with the devisement of methods that discretize carefully both topological laws and constitutive relations, bringing to the field of unstructured meshes the advantages of a correct topological time stepping. In particular, the joint use of error-based discretization strategies for the constitutive relations along with topological time-stepping schemes seems a promising and as yet unexplored field of enquiry.

As anticipated in the Introduction, we have not considered questions such as the rate of convergence, the stability, and the error analyses of the methods. In light of the present discussion it is, however, worth making at least one observation: The tendency of the various approaches toward the adoption of a number of common ideas includes the use of global variables associated with geometric objects for the discrete representation of fields. Therefore, it seems logical to also focus the error analyses on the global quantities, instead of applying these analyses to the local quantities that are reconstructed from global ones once the numerical problem has been solved. The error deriving from this last step is one of cochain-based field function approximation, and is derived from a process of reconstruction of the field functions, which starts from the aforementioned global values. This error is obviously relevant to the solution of physical field problems, but it can be considered separately from the previous phases of the numerical method. For example, the final field reconstruction can be conducted with different criteria with respect to possible reconstructions which took place during the discretization phase.

Finally, the emphasis placed in this study on a discrete approach to the modeling is not intended to indicate that the alternative continuous approaches should be abandoned in the near future or considered "bad" approaches. These alternative approaches are needed today and will continue to be in the future, since a discrete approach modeled on the reference discretization strategy presented in this work places some constraint on the relation between the problem to be solved and the resources required to actually do this. There will always be cases in which a solution is sought for which the discrete approach presented here appears in a particular moment not to be feasible. Returning to the theme of the Introduction, since good numerical mathematics is also the 
art of the possible, to cover these cases the ingenuity of the mathematician will be required to produce for these problems a formulation that is numerically feasible. Usually this requires embedding in the discrete formulation of a problem the physical and mathematical knowledge available regarding the problem and the general behavior of the solution. This includes the exploitation of the properties of the continuous mathematical model. We see in our times examples of this in spectral methods and compact finite difference methods applied to fluid dynamics. However, as time goes by, we can expect that the approach to the discrete formulation of field problems outlined in the present work will be found to be numerically manageable for a steadily widening range of problems, and that for these cases this approach will be recognized and adopted as the method of choice.

\section{CODA}

A first version of this work was published as Mattiussi (2000) and is presented here with minor corrections, some additions (mainly in the attempt to improve readability and to clarify some points that readers of the previous version found obscure), and a slightly different emphasis on some topics.

The response to the first appearance of this material [and to that of a previous paper dealing with similar issues but with greater attention to implementation details, in particular for what concerns the optimization of the reconstructionprojection process (Mattiussi, 1997)] made me feel as if only one part of the message that it was trying to convey was getting through, namely, the part concerning the possibility to reinterpret and compare the workings of existing numerical methods. This was probably a result of the quantity of material devoted to the analysis of some popular methods in light of the reference discretization strategy.

In fact, the possibilities opened by the application of algebraic topology and of the structural analysis of physical theories to the discretization of field problems go way beyond that. For example, they include the development of the reference discretization strategy introduced in this work, which-abstracting from the particular physical theory for which it is presented here, and thinking instead in terms of the factorization diagram - can be used as a template for the systematic discretization of generic field problems (and, therefore, for the development of new methods for the numerical solution of these problems) complying with the structure of the underlying physical theory. In this respect, a lot of work remains to be done to apply this approach to field problems for which the computational community is striving to improve the numerical solutions (in particular, if the improvements have to do with the physical soundness of the solutions). 
In relation to the history of exterior algebra, Gian Carlo Rota (1997) once wrote:

Evil tongues whispered that there was really nothing new in Grassmann's exterior algebra. ... The standard objection was expressed by the notorious question, "What can you prove with exterior algebra that you cannot prove without it?" Whenever you hear this question raised about some piece of new mathematics, be assured that you are likely to be in the presence of something important.... A proper retort might be: "You are right. There is nothing in yesterday's mathematics that you can prove with exterior algebra that could not also be proved without it. Exterior algebra is not meant to prove old facts, it is meant to disclose a new world" (pp. 47-48).

I hope that the publication of this contribution in its present form can help to convey better the neglected part of its message, namely, that, in its most ambitious embodiment, the application of the analysis of the structure of physical theories to the discretization of field problems is not meant to reinterpret old methods; it is meant to disclose a new world.

\section{REFERENCES}

Abraham, R., Marsden, J. E., and Ratiu, T. (1988). Manifolds, Tensor Analysis, and Applications. Berlin: Springer-Verlag.

Ahagon, A., Fujiwara, K., and Nakata, T. (1996). Comparison of various kinds of edge elements for electromagnetic field analysis. IEEE Trans. Magn. 32, 898-901.

Albanese, R., Fresa, T., Martone, R., and Rubinacci, G. (1994). An error based approach to the solution of full Maxwell equations. IEEE Trans. Magn. 30, 2968-2971.

Albanese, R., and Rubinacci, G. (1993). Analysis of three-dimensional electromagnetic fields using edge elements. J. Comput. Phys. 108, 236-245.

Albanese, R., and Rubinacci, G. (1998). Finite element methods for the solution of 3D eddy current problems, in Advances in Imaging and Electron Physics, Vol. 102, edited by P. Hawkes. Boston: Academic Press, pp. 1-86.

Baldomir, D., and Hammond, P. (1996). Geometry of Electromagnetic Systems. Oxford, UK: Oxford Univ. Press.

Bamberg, P., and Sternberg, S. (1988). A Course in Mathematics for Students of Physics: 1-2. Cambridge, UK: Cambridge Univ. Press.

Bateson, G. (1972). The logical categories of learning and communication, in Steps to an Ecology of Mind. New York: Ballantine Books.

Bellman, R. (1968). Some Vistas of Modern Mathematics. Univ. of Kentucky Press.

Belytschko, T., Krongauz, Y., Organ, D., Fleming, M., and Krysl, P. (1996). Meshless methods: an overview and recent developments. Comput. Methods Appl. Mechanics Eng. 139, 3-47.

Berenger, J. P. (1994). A perfectly matched layer for the absorption of electromagnetic waves. J. Comput. Phys. 114, 185-200.

Birss, R. R. (1980). Multivector analysis I-II. Phys. Lett. 78A, 223-230.

Bishop, R. L., and Goldberg, S. I. (1980). Tensor Analysis on Manifolds. New York: Dover. 
Bodenmann, R. (1995). Summation by Parts Formula for Noncentered Finite Differences. Research Report 95-07, Seminar für Angewandte Mathematik, ETH Zürich.

Boothby, W. M. (1986). An Introduction to Differentiable Manifolds and Riemannian Geometry, 2nd ed. San Diego: Academic Press.

Bossavit, A. (1998a). Computational Electromagnetism. San Diego: Academic Press.

Bossavit, A. (1998b). How weak is the "weak solution" in finite element methods? IEEE Trans. Magn. 34, 2429-2432.

Bowden, K. (1990). On general physical systems theories. Int. J. Gen. Syst. 18, 61-79.

Branin, F. H., Jr. (1966). The Algebraic-Topological Basis for Network Analogies and the Vector Calculus. Paper presented at the Symposium on Generalized Networks, Polytechnic Institute of Brooklyn, New York.

Burke, W. L. (1985). Applied Differential Geometry. Cambridge, UK: Cambridge Univ. Press.

Burnett, D. S. (1987). Finite Element Analysis. Reading, MA: Addison-Wesley.

Cartan, E. (1922). Le Çons sur les invariants intégraux. Paris: Hermann.

Cendes, Z. J. (1991). Vector finite elements for electromagnetic field computation. IEEE Trans. Magn. 27, 3958-3966.

Choquet-Bruhat, Y., and DeWitt-Morette, C. (1977). Analysis, Manifolds and Physics. Amsterdam: North-Holland.

de Rham, G. (1931). Sur l'Analysis situs des variétés à $n$ dimensions. J. Math. 10, 115-199.

de Rham, G. (1960). Variétés Différentiables. Paris: Hermann.

Deschamps, G. A. (1981). Electromagnetics and differential forms. Proc. IEEE 69(6), 676-696.

Dezin, A. A. (1995). Multidimensional Analysis and Discrete Models. Boca Raton, FL: CRC Press.

Dolcher, M. (1978). Algebra Lineare. Bologna: Zanichelli.

Eilenberg, S., and Steenrod, N. (1952). Foundations of Algebraic Topology. Princeton, NJ: Princeton Univ. Press.

Ferziger, J. H., and Perić, M. (1996). Computational Methods for Fluid Dynamics. Berlin: Springer-Verlag.

Flanders, H. (1989). Differential Forms with Applications to the Physical Sciences. New York: Dover.

Fletcher. C. A. J. (1984). Computational Galerkin Methods. Berlin: Springer-Verlag.

Franz, W. (1968). Algebraic Topology. New York: Ungar.

Golias, N. A., Tsiboukis, T. D., and Bossavit, A. (1994). Constitutive inconsistency: rigorous solution of Maxwell equations based on a dual approach. IEEE Trans. Magn. 30, 3586-3589.

Guibas, L., and Stolfi, J. (1985). Primitives for the manipulation of general subdivisions and the computation of Voronoi diagrams. ACM Trans. Graphics 4, 74-123.

Gustafsson, B., Kreiss, H.-O., and Oliger, J. (1995). Time Dependent Problems and Difference Methods. New York: Wiley.

Hocking, J. G., and Young, G. S. (1988). Topology. New York: Dover.

Hurewicz, W., and Wallman, H. (1948). Dimension Theory. Princeton, NJ: Princeton Univ. Press.

Hyman, J. M., and Shashkov, M. (1997). Natural discretization for the divergence, gradient, and curl on logically rectangular grids. Comput. Math. Applic. 30, 81-104.

Hyman, J. M., and Shashkov, M. (1999). Mimetic discretization for Maxwell's equations and equations of magnetic diffusion. J. Comput. Phys. 151, 881-909.

Isham, C. J. (1989). Modern Differential Geometry for Physicists. Singapore: World Scientific. Jackson, J. D. (1975). Classical Electrodynamics. New York: Wiley.

Jin, J. (1993). The Finite Element Method in Electromagnetics. New York: Wiley.

Kunz, K. S., and Luebbers, R. J. (1993). Finite Difference Time Domain Method for Electromagnetics. Boca Raton, FL: CRC Press.

Lebesgue, H. (1973). LeÇons sur l'Intégration. New York: Chelsea. 
Lee, J., Lee, R., and Cangellaris, A. (1997). Time-domain finite element methods. IEEE Trans. Antennas Propagat. 45, 430-442.

Lele, S. K. (1992). Compact finite difference schemes with spectral-like resolution. J. Comput. Phys. 103, 16-42.

Lilek, Z., and Perić, M. (1995). A fourth-order finite volume method with colocated variable arrangement. Comput. Fluids 24, 239-252.

MacLane, S. (1986). Mathematics Form and Function. Berlin: Springer-Verlag.

Madsen, N. K. (1995). Divergence preserving discrete surface integral methods for Maxwell's curl equations using non-orthogonal unstructured grids. J. Comput. Phys. 119, 34-45.

Marmin, F., Clénet, S., Pirou, F., and Bussy, P. (1998). Error estimation of finite element solution in non-linear magnetostatic 2D problems. IEEE Trans. Magn. 34, 3268-3271.

Mattiussi, C. (1997). An analysis of finite volume, finite element, and finite difference methods using some concepts from algebraic topology. J. Comput. Phys. 133, 289-309.

Mattiussi, C. (1998). Edge elements and cochain-based field function approximation, in Proceedings of the Fourth International Workshop on Electric and Magnetic Fields, Marseilles (France). pp. 301-306.

Mattiussi, C. (2000). The finite volume, finite element, and finite difference methods as numerical methods for physical field problems. Adv. Imaging Electron Phys. 113, 1-146 (P. Hawkes, Ed.).

Mattiussi, C. (2001). The geometry of time-stepping. Prog. Electromagn. Res. 32, 123-149.

Maxwell, J. C. (1871). Remarks on the mathematical classification of physical quantities. Proc. London Math. Soc. 3, 224-232.

Maxwell, J. C. (1884). Traité Élémentaire d'Électricité. Paris: Gauthier-Villars.

Misner, C. W., Thorne, K. S., and Wheeler, J. A. (1970). Gravitation. New York: Freeman.

Moore, W. (1989). Schrödinger, Life and Thought. Cambridge, UK: Cambridge Univ. Press.

Mur, G. (1994). Edge elements, their advantages and their disadvantages. IEEE Trans. Magn. 30, 3552-3557.

Nguyen, D. B. (1992). Relativistic constitutive relations, differential forms, and the $p$-compound. Am. J. Phys. 60, 1134-1144.

Oden, J. T. (1973). Finite element applications in mathematical physics, in The Mathematics of Finite Elements and Applications, edited by J. R. Whiteman. San Diego: Academic Press, pp. 239-282.

Oden, J. T., and Reddy, J. N. (1983). Variational Methods in Theoretical Mechanics, 2nd ed. Berlin: Springer-Verlag,

Oñate, E., and Idelsohn, S. R. (1992). A comparison between finite element and finite volume methods in CFD. Comput. Fluid Dynamics 1, 93.

Palmer, R. S., and Shapiro, V. (1993). Chain models of physical behavior for engineering analysis and design. Computer Science Technical Report TR93-1375, Cornell University, New York.

Penman, J. (1988). Dual and complementary variational techniques for the calculation of electromagnetic fields. Adv. Electron. Electron Phys. 70, 3I5-364 (P. Hawkes, Ed.).

Post, E. (1997). Formal Structure of Electromagnetics. General Covariance and Electromagnetics. New York: Dover.

Remacle, J.-F., Geuzaine, C., Dular, P., Hedia, H., and Legros, W. (1998). Error estimation based on a new principle of projection and reconstruction. IEEE Trans. Magn. 34, 3264-3267.

Rikabi, J., Bryant, C. F., and Freeman, E. M. (1988). An error-based approach to complementary formulations of static field solutions. Int. J. Numer. Methods Eng. 26, 1963-1987.

Rota, G. C. (1997). Combinatorics, Representation Theory and Invariant Theory pp. 39-54. Indiscrete Thoughts, edited by F. Palombi. Boston: Birkhäuser.

Schouten, J. A. (1989). Tensor Analysis for Physicist. New York: Dover.

Schroeder, W., and Wolff, I. (1994). The origin of spurious modes in numerical solutions of electromagnetic field eigenvalue problems. IEEE Trans. Microwave Theory Tech. 42, 644-653. 
Schutz, B. (1980). Geometrical Methods of Mathematical Physics. Cambridge, UK: Cambridge Univ. Press.

Shashkov, M. (1996). Conservative Finite-Difference Methods on General Grids. Boca Raton, FL: CRC Press.

Shashkov, M., and Steinberg, S. (1995). Support-operator finite-difference algorithms for general elliptic problems. J. Comput. Phys. 118, 131-151.

Strand, B. (1994). Summation by parts for finite difference approximation for $d / d x$. J. Comput. Phys. 110, 47-67.

Sun, D., Manges, J., Yuan, X., and Cendes, Z. (1995). Spurious modes in finite-element methods. IEEE Antennas Propagat. Mag. 37, 12-24.

Taflove, A. (1995). Computational Electrodynamics. The Finite-Difference Time-Domain Method. Boston: Artech House.

Taflove, A. (1998). Advances in Computational Electrodynamics. The Finite-Difference TimeDomain Method. Boston: Artech House.

Tarhasaari, T., Kettunen, L., and Bossavit, A. (1999). Some realizations of a discrete Hodge operator: a reinterpretation of finite element techniques. IEEE Trans. Magn. 35, 1494-1497.

Teixeira, F. L., and Chew, W. C. (1999a). Differential forms, metrics and the reflectionless absorption of electromagnetic waves. J. Electromagn. Wave 13, 665-686.

Teixeira, F. L., and Chew, W. C. (1999b). Lattice electromagnetic theory from a topological viewpoint. J. Math. Phys. 40, 169-187.

Tonti, E. (1975). On the Formal Structure of Physical Theories. Milano: Consiglio Nazionale delle Ricerche.

Tonti, E. (1976a). The reason for analogies between physical theories. Appl. Math. Modelling 1, $37-50$.

Tonti. E. (1976b). Sulla struttura formale delle teorie fisiche, in Rendiconti del Seminario Matematico e Fisico di Milano, Vol. XLVI. pp. 163-257.

Tonti, E. (1998). Algebraic topology and computational electromagnetism, in Proceedings of the Fourth International Workshop on Electric and Magnetic Fields, Marseilles (France). pp. 285-294.

Truesdell, C., and Noll, W. (1965). The non-linear field theories of mechanics, in Handbuch der Physik, Vol. 3/3, edited by S. Flugge. Berlin: Springer-Verlag.

Truesdell, C., and Toupin, R. A. (1960). The classical field theories, in Handbuch der Physik, Vol. 3/1, edited by S. Flugge. Berlin: Springer-Verlag, pp. 226-793.

Versteeg, H. K., and Malalasekera, W. (1995). An Introduction to Computational Fluid Dynamics. The Finite Volume Method. Harlow, England: Longman.

Warnick, K. F., Selfridge, R. H., and Arnold, D. V. (1997). Teaching electromagnetic field theory using differential forms. IEEE Trans. Educ. 40, 53-68.

Webb, J. P. (1993). Edge elements and what they can do for you. IEEE Trans. Magn. 29, $1460-1465$.

Weiland. T. (1984). On the numerical solution of Maxwell's equations and applications in the field of accelerator physics. Particle Accelerators 15, 245-292.

Weiland, T. (1996). Time domain electromagnetic field computation with finite difference methods. Int. J. Numer. Modelling 9, 295-319.

Whitney, H. (1957). Geometric Integration Theory. Princeton, NJ: Princeton Univ. Press. 